\title{
Computed Isotopic Inventory and Dose Assessment for SRS Fuel and Target Assemblies
}

by

M. C. Chandler

Westinghouse Savannah River Company

Savannah River Site

Aiken, South Carolina 29808

E T Kelusiky

SAIC

D C Thoman

SAIC

DOE Contract No. DE-AC09-89SR18035

This paper was prepared in connection with work done under the above contract number with the U.S.

Department of Energy. By acceptance of this paper, the publisher and/or recipient acknowledges the U.S. Government's right to retain a nonexclusive, royalty-free license in and to any copyright covering this paper, along with the right to reproduce and to authorize others to reproduce all or part of the copyrighted paper. 


\section{DISCLAMIER}

Portions of this document may be illegible in electronic image products. Images are produced from the best available original document. 


\section{DISCLAIMER}

This report was prepared as an account of work sponsored by an agency of the United States Government. Neither the United States Government nor any agency thereof, nor any of their employees, makes any warranty, express or implied, or assumes any legal liability or responsibility for the accuracy, completeness, or usefulness of any information, apparatus, product, or process disclosed, or represents that its use would not infringe privately owned rights. Reference herein to any specific commercial product, process, or service by trade name, trademark, manufacturer, or otherwise does not necessarily constitute or imply its endorsement, recommendation, or favoring by the United States Government or any agency thereof. The views and opinions of authors expressed herein do not necessarily state or reflect those of the United States Government or any agency thereof.

This report has been reproduced directly from the best available copy.

Available to DOE and DOE contractors from the Office of Scientific and Technical Information, P.O. Box 62, Oak Ridge, TN 37831; prices available from (615) 57.6-8401.

Available to the public from the National Technical Information Service, U.S. Department of Commerce, 5285 Port Royal Road, Springfield, VA 22161. 
HIGH LEVEL WASTE ENGINEERING

HLW ENGINEERING SUPPORT

COMPUTED ISOTOPIC INVENTORY

AND DOSE ASSESSMENT

FOR SRS FUEL AND TARGET ASSEMBLIES

By

M. C. Chandler, WSRC

E. T. Ketusky, SAIC

D. C. Thoman, SAIC

ISSUED: June 19, 1995
WSRC-TR-94-0456

REVISION: 0

KEYWORDS:

High level waste tanks, Radionuclide distributions, Dose contributions, Radioactive inventories

RETENTION:

PERMANENT

CLASSIFICATION: U Does Not Contain UCNI Damesialeat destee
M. C. Chandler, Author, HLWE (Document Sponsor)

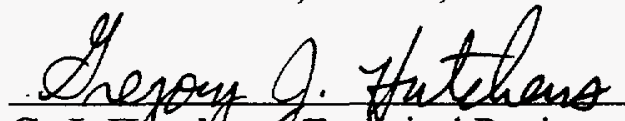

G. J. Hatcheng, Technical Reviewer, HLWE

\section{Clic l- Booxth}

C. I. ApontegTechnical Reviewer, HLWE

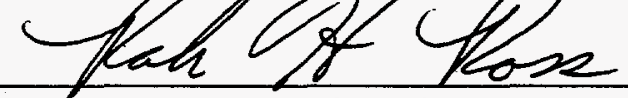

R. H. Ross, Technical Reviewer, HLWE

\section{Dames Paleut Weste}

J. R. Frester, Technical Reviewer, HLWE

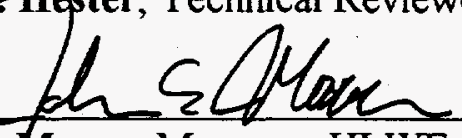

J. E. Marra, Manager, HLWE Support

Sollickert for

T. M. Monahon, Manager, HLWE
Date: $6 / 21 / 95$

Date: $6 / 20 / 95$

Date: $6 / 20 / 95$

Date: $6 / 20 / 95$

Date: $6 / 20 / 95$

Date: $6 / 22 / 95$

Date: $7 / 10 / 95$ 
WSRC-TR-94-0456

Revision 0

Page 2 of 56

\section{INTRODUCTION}

Past studies have identified and evaluated important radionuclide contributors to dose from reprocessed spent fuel sent to waste for Mark 16B and 22 fuel assemblies and for Mark 31A and $31 \mathrm{~B}$ target assemblies (References 8 - 11). Fission-product distributions after a 5- and 15-year decay time were calculated for a "representative" set of irradiation conditions (i.e., reactor power, irradiation time, and exposure) for each type of assembly (Reference 12). The numerical calculations were performed using the SHIELD/GLASS system of codes (References 2 - 6).

The sludge and supernate source terms for dose were studied separately with the significant radionuclide contributors for each identified and evaluated (References 8 - 11). Dose analysis considered both inhalation and ingestion pathways: The inhalation pathway was analyzed for both evaporative and volatile releases. Analysis of evaporative releases utilized release fractions for the individual radionuclides as defined in the ICRP-30 by DOE guidance (Reference 13). A release fraction of unity was assumed for each radionuclide under volatile-type releases, which would encompass internally initiated events (e.g., fires, explosions), process-initiated events (e.g., spills, improper transfer of materials), and externally initiated events (e.g., floods, earthquakes). Radionuclides which contributed at least $1 \%$ to the overall dose were designated as significant contributors.

The present analysis extends and complements the past analyses through considering a broader spectrum of fuel types and a wider range of irradiation conditions. The results provide for a more thorough understanding of the influences of fuel composition and irradiation parameters (i.e., reactor power, irradiation time, and exposure) on fission product distributions (at 2 years or more). Additionally, the present work allows for a more comprehensive evaluation of radionuclide contributions to dose and an estimation of the variability in the radionuclide composition of the dose source term that results from the spent fuel sent to waste encompassing a broad spectrum of fuel compositions and irradiation conditions.

\section{SUMMARY}

This report analyzes the variability of fission product distributions in waste sludge from reprocessed Savannah River Site (SRS) spent fuels and targets. Distributions are analyzed for various time periods (in the range of $2-20$ years) following irradiation in SRS reactors. The analyses of this report utilize numerical calculations of fission product and actinide quantities in spent fuels and targets, which were performed (Reference 1) using the SHIELD/GLASS codes (References 2 - 6) over a wide spectrum of fuel compositions and irradiation conditions that are representative of the history of reactor operations at SRS (Reference 7).

Calculations which are documented in this report involve application of reprocessing separation factors to fission products and actinides to determine relative quantities of these radionuclides sent to waste. Through the application of solubility data, estimates of radionuclide composition in the sludge portion of the waste are determined (supernate radionuclide compositions are not studied). These numerical estimates of sludge composition are then compared to available tank 
WSRC-TR-94-0456

Revision 0

Page 3 of 56

inventory data which provide estimates of selected sludge constituents (e.g., plutonium and uranium isotopes) based largely upon accountability records of the reprocessing facilities. Best agreement was observed when the waste in the tanks originated from fuel reprocessed in the Canyons within the last 15 to 20 years, came from the HHW stream, and contained no offsite fuel.

The impact of radionuclide variability (due to influences of variable fissile material composition, exposure, reactor power, and time since irradiation) on dose is also addressed in this report. The results of analyses showed the following list of radionuclides to be the important contributors to dose: Sr-90, Ru-106, Cs-137, Ce-144, Pm-147, Pu-238, Pu-239, Pu-240, and Pu-241.

\section{CONCLUSIONS}

Source dose calculations for waste sludge were performed for both fuel assemblies undergoing $\mathrm{HM}$ processing and target assemblies undergoing PUREX processing. The results confirm those of past analyses in identifying the following radionuclides, after a four-year decay time, as contributing significantly to dose (i.e., contributing at least $1 \%$ of the inhalation or ingestion dose): Sr-90, Ru-106, Cs-137, Ce-144, Pm-147, Pu-238, Pu-239, Pu-240, and Pu-241 (Am-241 which has been was identified as an important contributor in the past was not analyzed due to its unavailability from the SHIELD/GLASS results used here). The analysis exceeds those of the past through the consideration of a larger variety of fuel types and a wider range of irradiation conditions.

Also, the feasibility of using the output of SHIELD/GLASS results to model radionuclide distributions in the tank farms was investigated. HM processing of spent fuel assemblies was considered since radionuclide data is more readily available for sludge sent to waste from the HM process than it is for the PUREX process. The uranium and plutonium isotopic content of monthly sludge deposits made to the individual tanks of the tank farms has been estimated and documented in Reference 16. These inventory estimates were based upon accountability records (which rely on process control samples) and estimations based upon process data and knowledge. Best agreement between the numerical predictions and the inventory data was achieved when the inventory records showed that the waste deposits occurred within the last 15 to 20 years and had no offsite fuel mixed in (waste could be considered 100\% Mark 16B and 22 spent fuel) and came from the HHW stream (separation factors used in the calculations are based on that stream).

\section{RECOMMENDATIONS}

The lack of agreement in some cases between the tank inventory data and the numerical predictions suggests areas in which the prediction methodology can be upgraded with future work. Modeling of the composition of the sludge in the waste tanks could be advanced and improved by the following steps: (1) development of a database which tracks fuel (including offsite) that has been processed in $\mathrm{F}$ and $\mathrm{H}$ Canyons on a monthly basis providing information on fuel type, quantity, and irradiation conditions (reactor power and exposure); (2) development of separation fractions for the LHW stream; (3) execution of additional SHIELD/GLASS runs if necessary to cover a broader variety of fuels and wider range of exposures; and (4) development 
of a database which identifies for each batch of fuel processed and sent to waste the most appropriate SHIELD/GLASS results to use for modeling purposes. Note also that the separation factors (Reference 12) presently available and used in this report are based upon averages observed in the separation canyons in $1976-1977$. The variability of separation factors over the lifetime of separation operations should be investigated and incorporated into the analysis, if necessary.

\section{DISCUSSION}

\section{Backgound}

The SHIELD/GLASS codes were used in Reference 1 to calculate the production of radionuclides in SRS reactor assemblies and their decay over a period of time. Various charge types encompassing different types of fuel and a variety of lattice arrangements were chosen for analysis to reflect a broad range of reactor operations for the purpose of assessing impact on variability in radionuclide compositions. For each charge type, calculations of radionuclide quantities in the spent fuels and targets were performed over a wide range of reactor powers, exposures and times since irradiation. This set of data is analyzed in the present work to assess fission product variability.

To determine estimates of the radionuclide composition in the sludge waste following reprocessing of the spent fuels and targets, it was necessary to apply separation fractions and solubility partitions to the radionuclide data of Reference 1.

Separation factors signify the percent loss (either intentionally or unintentionally) of the various radionuclides from spent fuel to high level waste. Two sets of separation factors are listed below for the two major separation campaigns that have occurred over the history of canyon reprocessing operations, namely, the PUREX and HM campaigns. The PUREX process is used to separate plutonium from uranium; the HM process is used to recover uranium and byproduct neptunium.

Table 1 Radionuclide Separation Fractions (Reference 12)

\begin{tabular}{|c|c|c|}
\hline & \multicolumn{2}{|c|}{ Percent Loss to Waste } \\
\hline Element & PUREX & HM \\
\hline Tritium & 28 & 28 \\
\hline Nable Gases & 0 & 0 \\
\hline Uranium & 0.02 & 0.15 \\
\hline Neptunium & 6.0 & 3.5 \\
\hline Plutonium & 0.22 & 100 \\
\hline Am, Cm, etc., & 100 & 100 \\
\hline Fission Products & 100 & 100 \\
\hline
\end{tabular}


Fresh waste from the canyon reprocessing operations is sent to tanks in $\mathbf{F}$ and $\mathbf{H}$ Area for storage in the form of sludge and supernate. The solubility fraction of a radionuclide represents the fraction of the total radionuclide quantity that is soluble and is thus present in the supernate with the remainder in the sludge portion. Solubility fractions (associated with the 12 radionuclides shown to have a significant impact on dose) are listed in the following table.

Table 2 Solubility Fractions (Reference 14)

\begin{tabular}{|c|c|}
\hline Radionuclide & Solubility Fraction \\
\hline Sr-90 & $2.5 \mathrm{e}-04$ \\
\hline Ru-106 & 0.5 \\
\hline Cs-137 & 0.95 \\
\hline Ce-144 & $1.0 \mathrm{e}-04$ \\
\hline Pm-147 & $1.0 \mathrm{e}-04$ \\
\hline $\mathrm{Pu}-239$ & $3.0 \mathrm{e}-04$ \\
\hline $\mathrm{Pu}-240$ & $3.0 \mathrm{e}-04$ \\
\hline $\mathrm{Pu}-241$ & $3.0 \mathrm{e}-04$ \\
\hline $\mathrm{Pu}-238$ & $3.0 \mathrm{e}-04$ \\
\hline
\end{tabular}

The application of the separation fractions and solubility fractions (actually, multiplication of the solubility complement) to the radionuclide compositions calculated numerically for spent fuel and target assemblies yielded predicted radionuclide compositions for sludge waste.

The dose conversion factors (Reference 13) convert the radionuclide composition values to dose values. Separate dose conversion factors exist for each of the various dose pathways. Inhalation and ingestion dose conversion factors for the radionuclides of concern are shown in the table below. Also shown are release fractions which are needed to adjust the inhalation dose conversion factors under evaporative conditions.

Table 3 Dose Conversion Factors (DCFs) and Release Fractions (Derived from Reference 13)

\begin{tabular}{|c|c|c|c|}
\hline Radionuclide & $\begin{array}{c}\text { Ingestion DCF } \\
{[\text { Rem } / \mathrm{g}]}\end{array}$ & $\begin{array}{c}\text { Inhalation DCF } \\
{[\text { Rem } / \mathrm{g}]}\end{array}$ & Release Fraction \\
\hline $\mathrm{Sr}-90$ & $1.81 \mathrm{e}+07$ & $1.81 \mathrm{e}+08$ & 0.001 \\
\hline $\mathrm{Ru}-106$ & $7.03 \mathrm{e}+07$ & $1.47 \mathrm{e}+09$ & 0.01 \\
\hline $\mathrm{Cs}-137$ & $4.33 \mathrm{e}+06$ & $2.77 \mathrm{e}+06$ & 0.01 \\
\hline $\mathrm{Ce}-144$ & $6.38 \mathrm{e}+07$ & $1.12 \mathrm{e}+09$ & 0.001 \\
\hline $\mathrm{Pm}-147$ & $8.81 \mathrm{e}+05$ & $3.15 \mathrm{e}+07$ & 0.001 \\
\hline $\mathrm{Pu}-239$ & $2.67 \mathrm{e}+05$ & $3.16 \mathrm{e}+07$ & 0.001 \\
\hline $\mathrm{Pu}-240$ & $9.75 \mathrm{e}+05$ & $1.16 \mathrm{e}+08$ & 0.001 \\
\hline $\mathrm{Pu}-241$ & $8.86 \mathrm{e}+06$ & $1.03 \mathrm{e}+09$ & 0.001 \\
\hline $\mathrm{Pu}-238$ & $6.51 \mathrm{e}+07$ & $7.88 \mathrm{e}+09$ & 0.001 \\
\hline
\end{tabular}


WSRC-TR-94-0456

Revision 0

Page 6 of 56

\section{Results}

The SHIELD/GLASS results of Reference 1 were performed for four different fuel assemblies (Mark 5E, 12A, 16B, and 22 types) and three different target assemblies (Mark 31A, 31B, and $50 \mathrm{~A}$ types) over a broad spectrum of reactor conditions. The output from these numerical calculations served as input for a variety of radionuclide analyses that were performed and which are discussed below.

\section{Sludge Dose Contributions}

\section{HM Processing Contribution}

Past studies in the development of source terms for High Level Waste (HLW) have identified and evaluated important radionuclide contributors to dose from reprocessed spent fuel sent to waste for Mark 16B and 22 fuel assemblies and for Mark 31A and 31B target assemblies (References 8 11). These analyses, performed for a representative set of irradiation conditions for each type of . assembly, demonstrated that only a limited number of radionuclides contributed at least $1 \%$ or more to the total dose. The analysis presented in this section extends and complements the past work through the consideration of a larger variety of fuel types and a wider range of irradiation conditions. Dose contributions from only the sludge portion of waste are examined. Doses are studied for decay periods of 4 and 20 years (recall, data are available for $2,4,10$, and 20 years). These time frames encompass the 5- and 15-year decay periods examined in the past analyses.

Following the methodology of past work (References 811 ), the dose analysis will consider $\mathrm{HM}$ processing of spent fuel assemblies and PUREX processing of spent target assemblies. This approach is consistent with the general practices of reprocessing activities performed in the $F$ and H Canyons.

Starting from the computed radionuclide production data [units of grams per assembly] of the irradiated fuel, four major steps were performed to determine radionuclide contributions to dose: (1) multiplication by specific activity to arrive at radionuclide activity [Curies per assembly]; (2) multiplication by separation fractions (which represent percentages of total quantities of radionuclides sent to waste); (3) multiplication by "insolubility fractions" (which represent percentages of total quantities of radionuclides deposited in the sludge portion of the waste); and (4) multiplication by dose conversion factors [units of Rem per Curie] to convert activity levels to dose levels. The tabular data needed to perform these sludge dose calculations are documented in the spreadsheet in Appendix A.

Comprehensive dose analyses were performed for both the Mark 16B and 22 fuel assemblies in order to study the effects of irradiation conditions and decay time on dose contributions. Additional dose analyses for Mark $5 \mathrm{E}$ and 12A fuel assemblies allow for the study of the effects of fuel composition. The results are summarized in tables located in Appendix B. 
WSRC-TR-94-0456

Revision 0

Page 7 of 56

Taking into consideration both inhalation and ingestion pathways, the list of important contributors to dose (as defined as contributing $1 \%$ or more to the total) is the same as identified in References 8 and 9, except that Am-241 was not considered in the present analysis due to its unavailability from the SHIELD/GLASS results. (References 8 and 9 showed the relative contribution of Am-241 to the sludge source term from HM processing of fuel assemblies to be $3 \%$ or less.) The twelve radionuclides that contribute significantly to dose are those listed in Tables 2 and 3.

A more thorough examination of the results of the present work discloses useful information and well-defined trends. The relative contribution to dose of the significant radionuclides for various Mark 16B and 22 cases can be seen from the tables in Appendix B. The Pu-238 and Sr-90 radionuclides are seen to be the two most important contributors to dose for Mark 16B and 22 fuels; for example, these two radionuclides account for $80-90 \%$ of the volatile inhalation dose at 4 years (their contribution is slightly higher at 20 years). At a 20 -year decay time, the relative contribution to dose of Pu-238 increases due to its larger half life (i.e., 88 years) than Sr-90 (28 years).

The table in Appendix B also contains results from Mark 5E and 12A calculations. Note that the same radionuclides constitute the list of important contributors to dose for these fuels as for Mark $16 \mathrm{~B}$ and 22 fuels, although some significant differences are noticeable in relative contribution levels. For example, $\mathrm{Ce}-144$ is a more significant contributor to dose for Mark 12A fuel than it is for the other fuels. The $\mathrm{Sr}-90, \mathrm{Ce}-144$, and $\mathrm{Pu}-238$ radionuclides are observed to contribute over $80 \%$ of the volatile inhalation dose at 4 years for Mark $12 \mathrm{~A}$ fuel (true also at 20 years even though the contribution of Ce-144 is negligible at this time frame). For Mark .5E fuel, the plutonium isotopes of mass number 239, 240, and 241 are the dominant contributors to volatile inhalation dose, both at 4 and 20 years.

\section{PUREX Processing Contribution}

Sludge dose analysis was also performed for waste from spent target assemblies having undergone PUREX processing. The tabular data needed to perform the sludge dose calculations are documented in Appendix A.

Dose analyses were performed for Mark $31 \mathrm{~A}$ target assemblies in order to study the effects of irradiation conditions and decay time on dose contributions. The results are summarized in Appendix C.

Taking into consideration both inhalation and ingestion pathways, the list of important contributors to dose (as defined as contributing $1 \%$ or more to the total) is the same as identified in Reference 10, except that Am-241 was not considered in the present analysis due to its unavailability from the SHIELD/GLASS results. (Reference 10 showed the relative contribution of Am-24l to the sludge source term from PUREX processing of target assemblies to be as high as $28 \%$.) 
WSRC-TR-94-0456

Revision 0

Page 8 of 56

\section{HM Waste Tank Inventory Comparisons}

The feasibility of using SHIELD/GLASS results to model radionuclide distributions in the tank farms is investigated in this section of the report. HM processing of spent fuel assemblies will be considered since radionuclide data is more readily available for the HM process than it is for the PUREX process (Reference 15).

Specifically, the SHIELD/GLASS radionuclide masses are used to predict the relative distributions of uranium and plutonium isotopes sent to individual waste tanks in sludge form as documented by accountability records (based on process control samples) and estimations based upon process data and knowledge (Reference 15). The following radionuclides are inventoried in the individual tank sludge report (Reference 16): U-233, U-234, U-235, U-236, U-238, Np-237, PU-238, Pu-239, Pu-240, Pu-241, and Pu-242.

The distribution of these radionuclides in sludge waste as a function of reactor power, exposure, and decay time was determined from the SHIELD/GLASS output. This involved adjusting the SHIELD/GLASS output of radionuclide data through the appropriate use of solubility fractions and separation fractions (HM processing) to reflect radionuclide distributions in sludge waste.

The results of calculations are presented in Appendix D, Figures D-1 through D-6. Figures D-7 through D-24 present the comparisons between inventory data and numerical predictions. The results presented in these graphs are normalized by dividing the constituent mass by the sum of the constituent masses.

Figure D-1 contains the results from a sample Mark 22 case, which shows that the distribution of the inventoried radionuclides does not change significantly over the time period of 20 years due to the long half lives of these radionuclides. Noticeable changes of small magnitude are observed in Pu-238 (half life of 88 years) and Pu-241 (15 years).

Figure D-2 shows that power level for a given exposure does not have any significant influence on the distribution of the inventoried radionuclides. Thus, for our purposes, it is not necessary to be concerned in this section with the fact that fuel sent to waste has been irradiated over a wide range of reactor power levels over the history of site operations.

The influence of exposure level on the relative quantities of the inventoried radionuclides for Mark 22 fuel is shown in Figure D-3. The most noticeable feature is the decrease in U-235 content as exposure increases, which is as expected since Mark 22 charges involve the fissioning of U-235.

Figure D-4 is an analogous graph for Mark 16B fuel showing similar behavior. Also shown on the graph are the Mark 22 results at $80 \mathrm{MWD} / \mathrm{ft}$, which are seen to be very similar to those for Mark 16B fuel at $80 \mathrm{MWD} / \mathrm{ft}$. This is an important fact to remember since Mark 16Bs and Mark $22 \mathrm{~s}$ have been the only SRS fuel assemblies of significant quantity processed in the $\mathrm{H}$ Canyon since the late 70 s (References 15 and 17). Also, the $80 \mathrm{MWD} / \mathrm{ft}$ value is representative of typical 
WSRC-TR-94-0456

Revision 0

Page 9 of 56

exposures that the Mark 22s and Mark 16Bs have undergone (the analyses of Reference 12 used values of $80 \mathrm{MWD} / \mathrm{ft}$ and $95 \mathrm{MWD} / \mathrm{ft}$ for Mark 22 and Mark 16B exposures, respectively, as the basis for their calculations).

Figures D-5 and D-6 show distributions of radionuclides at various fission exposures for Mark 5E and 12A fuel assemblies. Since these two assemblies date back to the mid-to-late sixties when a wide variety of fuel assemblies were irradiated, they will not play a prominent role in the analyses that follow. For the sake of simplicity, comparisons of numerical calculations to tank inventories will largely be confined to tanks that have received waste from the $\mathrm{H}$ Canyon only during the past 20 years, and thus, they largely contain waste from processed Mark $16 \mathrm{~B}$ and 22 fuel assemblies (References 15 and 17). The Mark 5E and 12A data are included for completeness and to facilitate possible future analysis.

Reference 16 tracks monthly sludge deposits to individual tanks. Monthly data was extracted from that report for comparison with the numerical calculations of this report. Data which allowed for the most straightforward comparisons with the numerical calculations were extracted and investigated first. This data met the following criteria: (1) waste from the HM process; (2) waste from the high heat waste (HHW) stream (HM separation factors from Reference 12 and used in this report appear to apply to the HHW stream only); (3) waste sent to tanks within the last 20 years; and (4) the waste deposit does not contain offsite fuel (spreadsheet from Reference 15 contains a column for offsite fuel aluminum, so a nonzero entry in that column identifies the presence of offsite fuel in the waste deposit).

The inventory data shown in Figures D-7 through D-10 met this criteria and are shown with the results from the numerical calculations for Mark 16B fuel at three different exposures $(20,40$ and $80 \mathrm{MWD} / \mathrm{ft}$ ). In general, agreement is observed between the inventory data and the numerical predictions (typically, the results for $80 \mathrm{MWD} / \mathrm{ft}$ provide the best match with the inventory data). Note that in these graphs and in those that follow, the inventory data is plotted as column bars while the numerical predictions are shown as points connected by lines.

The second set of figures, Figures D-11 through D-16, show comparisons between numerical calculations (Mark 22 fuel) and tank contents which do not meet all four criterion points listed above. Specifically, the tank contents meet criteria (1) and (2), but not (4) which is the zero offsite fuel criterion, and not necessarily (3) which is the 20-year criterion. As expected, greater deviation is seen in general between the numerical calculations and the inventory data. For example, both significant underprediction of U-238 content and overprediction of Pu-239 content are observed for Tanks 32,35 , and 36 .

In the third set of figures, Figures D-17 through D-24, the inventory data are assured to meet only criterion (1), that is, HM processing. Poor agreement between the numerical predictions and tank inventory data is observed for Tanks 13,21,22, and 43. These tanks contain waste predominantly from the low heat waste (LHW) stream which has significantly different separation factors associated with it which accounts for the poor results. 
WSRC-TR-94-0456

Revision 0

Page 10 of 56

One last comparison was made, shown in Figure D-24, using the sludge waste deposit to Tank 15 made in December 1968. This deposit is from the HHW stream of HM processing and does not contain offsite fuel. Since the SRS fuel processed in this batch is Mark 12A (Reference 17), comparisons with numerical calculations were based on this fuel. There is good agreement between the numerical predictions for Mark 12A fuel at $40 \mathrm{MWD} / \mathrm{ft}$ exposure and the inventory data. 
WSRC-TR-94-0456

Revision 0

Page 11 of 56

\section{REFERENCES}

1. Webb, R. L., "Fission Product Production at SRS (U)", EPD-CTG-94-0039, December 1994.

2. Frost, R. L., "Validation Study for FPCALCG (U)", WSRC-TR-92-188, Westinghouse Savannah River Company, Savannah River Site, Aiken, SC, April 1992.

3. Finch, D. R., "The SHIELD System User's Manual (U)," DPSTM-87-700-2, November 1987.

4. Frost, R. L. and Finfrock, S. H., "GLASS User's Manual (U)", WSRC-TR-91-143, Westinghouse Savannah River Company, Savannah River Site, Aiken, SC, January 1992.

5. Graves, W. E., "GLASS User's Manual (U)," DPSTM-89-100-1, July 1989.

6. Graves, W. E. and Webb, R. L., "GLASS Code Validation (U)", WSRC-TR-91-002, Westinghouse Savannah River Company, Savannah River Site, Aiken, SC, January 1991.

7. Jarriel, J. L., "Reactor Operating History (U)", OPS-PSD-91-0032, March 1991.

8. Aponte, C. I., "Sludge Source Term (HM Process Inhalation Dose Impact) (U)", WSRCTR-94-0147, Westinghouse Savannah River Company, Savannah River Site, Aiken, SC, June 1994

9. Aponte, C. I., "Sludge Source Term (HM Process Ingestion Dose Impact) (U)", WSRCTR-94-0182, Westinghouse Savannah River Company, Savannah River Site, Aiken, SC, June 1994.

10. Aponte, C. I., "Sludge Source Term (PUREX Process Radionuclide Dose Impact) (U)", WSRC-TR-94-0262, Westinghouse Savannah River Company, Savannah River Site, Aiken, SC, June 1994.

11. Aponte, C. I., "Supernate Source Term Analysis (U)", WSRC-TR-94-0276, Westinghouse Savannah River Company, Savannah River Site, Aiken, SC, June 1994.

12. "Preliminary Technical Data Summary - Defense Waste Processing Facility - Stage 2", DPSTD-80-39, December 1980.

13. "Hazard Categorization and Accident Analysis Techniques for Compliance with DOE Order 5480.23, Nuclear Safety Analysis Reports," DOE-STD-1027-92, U. S. Department of Energy, Washington, DC, December 1992. 
WSRC-TR-94-0456

Revision 0

Page 12 of 56

14. Fowler, J. R., "Update of Chemical and Radiochemical Composition of Decontaminated Soluble Waste from the Precipitation Process," DPST-82-759, August 1982.

15. Cavin, W. S., "Separations Waste Sludge Spreadsheet (U)", WSRC-TR-93-135, Westinghouse Savannah River Company, Savannah River Site, Aiken, SC, July, 1993.

16. Chandler, M. C., "Estimated Sludge Inventory for Individual Tanks (U)", WSRC-TR-940191, Rev. 1, Westinghouse Savannah River Company, Savannah River Site, Aiken, SC, August 1994.

17. Pickett, C. E., "Plutonium Discards to Waste from H-Canyon (U)," OPS-STH-900326, June 29, 1992. 
WSRC-TR-94-0456

Revision 0

Page 13 of 56

Appendix A

Spreadsheet for Processing Calculations 
WSRC-TR-94-0456

Revision 0

Page 14 of 56

\begin{tabular}{|c|c|c|c|c|c|c|c|c|c|c|}
\hline \multirow[t]{2}{*}{ Nuclide } & \multirow[t]{2}{*}{$\begin{array}{l}\text { Atomic } \\
\text { Weight }\end{array}$} & \multirow{2}{*}{$\begin{array}{l}\text { Half Life } \\
\text { [s] }\end{array}$} & \multirow[t]{2}{*}{ Lambda } & \multirow{2}{*}{$\begin{array}{l}\text { Specific } \\
\text { Activity } \\
{[\mathrm{Ci} / g]}\end{array}$} & \multirow{2}{*}{$\begin{array}{l}\text { IRCP-30 } \\
\text { Inhal. } \\
\text { DCF } \\
\text { [rem/uCi] }\end{array}$} & \multirow{2}{*}{$\begin{array}{l}\text { Release } \\
\text { Fraction }\end{array}$} & \multirow{2}{*}{$\begin{array}{l}\text { IRCP-30 } \\
\text { Inges. } \\
\text { DCF } \\
\text { [rem/uCi] }\end{array}$} & \multirow{2}{*}{$\begin{array}{l}\text { Solubility } \\
\text { Fraction }\end{array}$} & \multicolumn{2}{|c|}{$\begin{array}{l}\text { Loss to Waste } \\
\text { Fraction }\end{array}$} \\
\hline & & & & & & & & & & PUREX \\
\hline$\overline{H 1}$ & 1.01 & & $0.00 \mathrm{E}+00$ & $0.00 \mathrm{E}+00$ & & & & & & \\
\hline H 2 & 2.01 & & $0.00 E+00$ & $0.00 E+00$ & & & & & & \\
\hline H 3 & 3.02 & $3.87 E+08$ & $1.79 E-09$ & $9.67 E+03$ & $9.50 \mathrm{E}-05$ & $1.00 E+00$ & $6.30 \mathrm{E}-05$ & $1.00 E+00$ & 0.2800 & 0.2800 \\
\hline HE 3 & 3.02 & & $0.00 E+00$ & $0.00 E+00$ & & & & & & \\
\hline HE 4 & 4.00 & & $0.00 E+00$ & $0.00 E+00$ & & & & & & \\
\hline LI 6 & 6.02 & & $0.00 E+00$ & $0.00 E+00$ & & & & & & \\
\hline LI 7 & 7.02 & & $0.00 E+00$ & $0.00 E+00$ & & & & & & \\
\hline GE 72 & 71.92 & & $0.00 E+00$ & $0.00 E+00$ & & & & & & \\
\hline GE 73 & 72.92 & & $0.00 E+\infty 0$ & $0.00 E+00$ & & & & & & \\
\hline GE 74 & 73.92 & & $0.00 \mathrm{E}+00$ & $0.00 E+00$ & & & & & & \\
\hline GE 76 & 75.92 & & $0.00 E+00$ & $0.00 E+00$ & & & & & & \\
\hline AS 75 & 74.92 & & $0.00 E+00$ & $0.00 E+00$ & & & & & & \\
\hline SE 77 & 76.92 & & $0.00 E+00$ & $0.00 E+00$ & & & & & & \\
\hline SE 78 & 77.92 & & $0.00 E+00$ & $0.00 E+00$ & & & & & & \\
\hline SE 79 & 78.92 & $2.05 E+12$ & $3.38 \mathrm{E}-13$ & 6.97E-02 & 8.90E-03 & $1.00 \mathrm{E}-02$ & 8.30E-03 & $1.00 E-01$ & 1.0000 & 1.0000 \\
\hline SE 80 & 79.92 & & $0.00 E+00$ & $0.00 E+00$ & & & & & & \\
\hline SE 82 & 81.92 & & $0.00 E+00$ & $0.00 E+00$ & & & & & & \\
\hline BR 81 & 80.92 & & $0.00 E+00$ & $0.00 E+00$ & & & & & & \\
\hline KR 82 & 81.91 & & $0.00 E+00$ & $0.00 E+00$ & & & & & & \\
\hline KR 83 & 82.91 & & $0.00 E+00$ & $0.00 E+00$ & & & & & & \\
\hline KR 84 & 83.91 & & $0.00 E+00$ & $0.00 E+00$ & & & & & & \\
\hline KR 85 & 84.91 & $3.39 E+08$ & 2.05E-09 & $3.92 E+02$ & & & & & & \\
\hline KR 86 & 85.91 & & $0.00 E+00$ & $0.00 E+00$ & & & & & & \\
\hline RB 85 & 84.91 & & $0.00 E+00$ & $0.00 E+00$ & & & & & & \\
\hline RB 87 & 86.91 & $1.48 E+18$ & $4.68 \mathrm{E}-19$ & $8.76 E-08$ & $3.30 E-03$ & $1.00 E-03$ & 4.80E-03 & $9.50 \mathrm{E}-01$ & 1.0000 & 1.0000 \\
\hline SR 88 & 87.91 & & $0.00 E+00$ & $0.00 E+00$ & & & & & & \\
\hline SR 89 & 88.91 & $4.49 E+06$ & $1.54 \mathrm{E}-07$ & $2.82 E+04$ & 3.70E-02 & $1.00 E-03$ & $8.70 E-03$ & $2.50 \mathrm{E}-04$ & 1.0000 & 1.0000 \\
\hline SR 90 & 89.91 & 8.87E+08 & $7.82 \mathrm{E}-10^{\circ}$ & $1.42 E+02$ & $1.30 E+00$ & $1.00 E-03$ & 1.30E-01 & 2.50E-04 & 1.0000 & 1.0000 \\
\hline Y 89 & 88.91 & & $0.00 \mathrm{E}+00$ & $0.00 E+00$ & & & & & & \\
\hline Y 90 & 89.91 & $2.31 E+05$ & $3.00 \mathrm{E}-06$ & $5.44 E+05$ & 8.20E-03 & $1.00 \mathrm{E}-03$ & $1.00 E-02$ & $2.50 E-04$ & 1.0000 & 1.0000 \\
\hline Y 91 & 90.91 & $5.06 \mathrm{E}+06$ & 1.37E-07 & $2.45 E+04$ & 4.40E-02 & $1.00 E-03$ & 8.90E-03 & $1.00 \mathrm{E}-04$ & 1.0000 & 1.0000 \\
\hline ZR 90 & 89.90 & & $0.00 E+00$ & $0.00 E+00$ & & & & & & \\
\hline ZR 91 & 90.91 & & $0.00 E+00$ & $0.00 E+00$ & & & & & & \\
\hline ZR 92 & 91.91 & & $0.00 E+00$ & $0.00 E+00$ & & & & & & \\
\hline ZR 93 & 92.91 & $3.00 E+13$ & 2.31E-14 & 4.05E-03 & $3.20 \mathrm{E}-01$ & $1.00 \mathrm{E}-03$ & $1.60 \mathrm{E}-03$ & $9.60 \mathrm{E}-06$ & 1.0000 & 1.0000 \\
\hline ZR 94 & 93.91 & & $0.00 E+00$ & $0.00 E+00$ & & & & & & \\
\hline ZR 95 & 94.91 & $5.66 \mathrm{E}+06$ & 1.22E-07 & $2.10 \mathrm{E}+04$ & $1.80 \mathrm{E}-02$ & $1.00 E-03$ & $3.40 \mathrm{E}-03$ & $1.00 \mathrm{E}-05$ & 1.0000 & 1.0000 \\
\hline ZR 96 & 95.91 & & $0.00 E+00$ & $0.00 E+00$ & & & & & & \\
\hline NB 95 & 94.91 & $3.03 E+06$ & $2.29 E-07$ & $3.92 E+04$ & 4.50E-03 & $1.00 E-03$ & $2.20 E-03$ & $1.00 \mathrm{E}-05$ & 1.0000 & 1.0000 \\
\hline NB 95M & 94.91 & 3.12E+05 & $2.22 E-06$ & $3.81 E+05$ & $2.20 E-03$ & $1.00 E-03$ & $2.00 E-03$ & $1.00 \mathrm{E}-05$ & 1.0000 & 1.0000 \\
\hline MO 95 & 94.91 & & $0.00 E+00$ & $0.00 E+00$ & & & & & & \\
\hline MO 96 & 95.90 & & $0.00 E+00$ & $0.00 E+00$ & & & & & & \\
\hline MO 97 & 96.91 & & $0.00 E+00$ & $0.00 E+00$ & & & & & & \\
\hline MO 98 & 97.91 & & $0.00 E+00$ & $0.00 E+00$ & & & & & & \\
\hline M0100 & 99.91 & & $0.00 E+00$ & $0.00 E+00$ & & & & & & \\
\hline TC 99 & 99.00 & $6.72 E+12$ & $1.03 E-13$ & $1.70 E-02$ & 7.50E-03 & $1.00 E-03$ & 1.30E-03 & 3.00E-01 & 1.0000 & 1.0000 \\
\hline RU100 & 99.90 & & $0.00 E+00$ & $0.00 E+00$ & & & & & & \\
\hline RU101 & 100.91 & & $0.00 E+00$ & $0.00 E+00$ & & & & & & \\
\hline
\end{tabular}


WSRC-TR-94-0456

Revision 0

Page 15 of 56

\begin{tabular}{|c|c|c|c|c|c|c|c|c|c|c|}
\hline \multirow[t]{2}{*}{ Nuclide } & \multirow[t]{2}{*}{$\begin{array}{l}\text { Atomic } \\
\text { Weight }\end{array}$} & \multirow{2}{*}{$\begin{array}{c}\text { Half Life } \\
{[\mathbf{s}]}\end{array}$} & \multirow[t]{2}{*}{ Lambda } & \multirow{2}{*}{$\begin{array}{c}\text { Specific } \\
\text { Activity } \\
\text { [Ci/g] }\end{array}$} & \multirow{2}{*}{$\begin{array}{l}\text { IRCP-30 } \\
\text { Inhal. } \\
\text { DCF } \\
\text { [rem/uCi] }\end{array}$} & \multirow{2}{*}{$\begin{array}{l}\text { Release. } \\
\text { Fraction }\end{array}$} & \multirow{2}{*}{$\begin{array}{l}\text { IRCP-30 } \\
\text { Inges. } \\
\text { DCF } \\
\text { [rem/uCi] }\end{array}$} & \multirow{2}{*}{$\begin{array}{l}\text { Solubility } \\
\text { Fraction }\end{array}$} & \multicolumn{2}{|c|}{$\begin{array}{l}\text { Loss to Waste } \\
\text { Fraction }\end{array}$} \\
\hline & & & & & & & & & $H M$ & PUREX \\
\hline RU102 & 101.90 & & $0.00 E+00$ & $0.00 E+00$ & & & & & & \\
\hline RU103 & 102.91 & $3.42 E+06$ & 2.03E-07 & $3.20 E+04$ & $7.80 \mathrm{E}-03$ & $1.00 E-02$ & $2.70 E-03$ & $5.00 \mathrm{E}-01$ & 1.0000 & 1.0000 \\
\hline RU104 & 103.91 & & $0.00 E+00$ & $0.00 E+00$ & & & & & & \\
\hline RU106 & 105.91 & $3.19 E+07$ & 2.17E-08 & $3.34 E+03$ & 4.40E-01 & $1.00 E-02$ & 2.10E-02 & $5.00 E-01$ & 1.0000 & 1.0000 \\
\hline $\mathrm{RH} 103$ & 102.91 & & $0.00 E+00$ & $0.00 E+00$ & & & & & & \\
\hline RH103M & 102.91 & $3.36 \mathrm{E}+03$ & $2.06 \mathrm{E}-04$ & $3.26 \mathrm{E}+07$ & & & & & & \\
\hline RH106 & 105.91 & 2.99E+01 & 2.32E-02 & $3.56 \mathrm{E}+09$ & & & & & & \\
\hline PD104 & 103.90 & & $0.00 E+00$ & $0.00 E+00$ & & & & & & \\
\hline PD105 & 104.91 & & $0.00 \mathrm{E}+00$ & $0.00 E+00$ & & & & & & \\
\hline PD106 & 105.90 & & $0.00 E+00$ & $0.00 E+00$ & & & & & & \\
\hline PD107 & 106.90 & $2.05 E+14$ & $3.38 E-15$ & 5.15E-04 & $1.30 \mathrm{E}-02$ & $1.00 \mathrm{E}-03$ & $1.40 E-04$ & 1.00E-04 & 1.0000 & 1.0000 \\
\hline PD108 & 107.90 & & $0.00 E+00$ & $0.00 E+00$ & & & & & & \\
\hline PD110 & 109.91 & & $0.00 E+00$ & $0.00 E+00$ & & & & & & \\
\hline AG109 & 108.90 & & $0.00 E+00$ & $0.00 E+00$ & & & & & & \\
\hline$A G 110$ & 109.91 & $2.18 E+07$ & $3.18 E-08$ & $4.71 E+03$ & & & & & & \\
\hline CD110 & 109.90 & & $0.00 E+00$ & $0.00 E+00$ & & & & & & \\
\hline CD111 & 110.90 & & $0.00 E+00$ & $0.00 E+00$ & & & & & & \\
\hline CD112 & 111.90 & & $0.00 E+00$ & $0.00 E+00$ & & & & & & \\
\hline CD113 & 112.90 & & $0.00 E+00$ & $0.00 E+00$ & & & & & & \\
\hline CD114 & 113.90 & & $0.00 E+00$ & $0.00 \mathrm{E}+00$ & & & & & & \\
\hline CD115M & 114.90 & $3.85 E+06$ & $1.80 E-07$ & $2.55 E+04$ & $6.50 \mathrm{E}-02$ & 1.00E-03 & $1.50 E-02$ & $5.00 \mathrm{E}-02$ & 1.0000 & 1.0000 \\
\hline CD116 & 115.90 & & $0.00 E+00$ & $0.00 E+00$ & & & & & & \\
\hline IN115 & 114.90 & $1.58 E+22$ & 4.40E-23 & $6.23 E-12$ & $3.40 \mathrm{E}+00$ & $1.00 E-03$ & $1.40 E-01$ & $5.00 \mathrm{E}-02$ & 1.0000 & 1.0000 \\
\hline IN115M & 114.90 & $1.62 E+04$ & 4.28E-05 & $6.06 E+06$ & & & & & & \\
\hline SN116 & 115.90 & & $0.00 E+00$ & $0.00 E+00$ & & & & & & \\
\hline SN117 & 116.90 & & $0.00 E+00$ & $0.00 E+00$ & & & & & & \\
\hline SN118 & 117.90 & & $0.00 E+00$ & $0.00 \mathrm{E}+00$ & & & & & & \\
\hline SN119 & 118.90 & & $0.00 \mathrm{E}+00$ & $0.00 E+00$ & & & & & & \\
\hline SN120 & 119.90 & & $0.00 \mathrm{E}+00$ & $0.00 \mathrm{E}+00$ & & & & & & \\
\hline SN121M & 120.90 & $1.58 \mathrm{E}+09$ & 4.40E-10 & $5.92 E+01$ & $8.90 \mathrm{E}-03$ & $1.00 E-03$ & $1.30 E-03$ & $4.00 \mathrm{E}-02$ & 1.0000 & 1.0000 \\
\hline SN122 & 121.90 & & $0.00 E+00$ & $0.00 E+00$ & & & & & & \\
\hline SN123 & 122.91 & $1.11 E+07$ & $6.22 E-08$ & $8.24 E+03$ & 3.00E-02 & $1.00 E-03$ & $7.70 E-03$ & 4.00E-02 & 1.0000 & 1.0000 \\
\hline SN124 & 123.91 & & $0.00 E+00$ & $0.00 E+00$ & & & & & & \\
\hline SN126 & 125.91 & $3.15 E+13$ & $2.20 \mathrm{E}-14$ & 2.84E-03 & $8.60 E-02$ & 1.00E-03 & $1.70 E-02$ & 4.00E-02 & 1.0000 & 1.0000 \\
\hline SB121 & 120.90 & & $0.00 E+00$ & $0.00 E+00$ & & & & & & \\
\hline SB123 & 122.90 & & $0.00 E+00$ & $0.00 E+00$ & & & & & & \\
\hline SB124 & 123.91 & $5.20 E+06$ & 1.33E-07 & $1.75 E+04$ & & & & & & \\
\hline SB125 & 124.90 & $8.62 E+07$ & 8.05E-09 & $1.05 E+03$ & $9.80 E-03$ & 1.00E-03 & $2.60 E-03$ & $6.00 E-03$ & 1.0000 & 1.0000 \\
\hline SB126 & 125.91 & $\cdot 1.07 E+06$ & $6.47 \mathrm{E}-07$ & $8.36 E+04$ & & & & & & \\
\hline SB126M & 125.91 & $1.14 E+03$ & $6.08 E-04$ & $7.86 E+07$ & & & & & & \\
\hline TE122 & 121.90 & & $0.00 E+00$ & $0.00 E+00$ & & & & & & \\
\hline TE124 & 123.90 & & $0.00 \mathrm{E}+00$ & $0.00 E+00$ & & & & & & \\
\hline TE125 & 124.90 & & $0.00 E+00$ & $0.00 E+00$ & & & & & & \\
\hline TE125M & 124.90 & $5.01 E+06$ & $1.38 \mathrm{E}-07$ & $1.80 E+04$ & & & & & & \\
\hline TE126 & 125.90 & & $0.00 E+00$ & $0.00 E+00$ & & & & & & \\
\hline TE127 & 126.90 & $3.37 E+04$ & 2.06E-05 & $2.64 \mathrm{E}+06$ & & & & & & \\
\hline TE127M & 126.90 & $9.42 E+06$ & 7.36E-08 & $9.44 E+03$ & 1.90E-02 & 1.00E-02 & $7.90 \mathrm{E}-03$ & $5.00 E-04$ & 1.0000 & 1.0000 \\
\hline TE128 & 127.90 & & $0.00 E+00$ & $0.00 E+00$ & & & & & & \\
\hline
\end{tabular}


WSRC-TR-94-0456

Revision 0

Page 16 of 56

\begin{tabular}{|c|c|c|c|c|c|c|c|c|c|c|}
\hline \multirow[t]{2}{*}{ Nuclide } & \multirow[t]{2}{*}{$\begin{array}{l}\text { Atomic } \\
\text { Weight }\end{array}$} & \multirow{2}{*}{$\begin{array}{l}\text { Half Life } \\
{[\mathbf{s}]}\end{array}$} & \multirow[t]{2}{*}{ Lambda } & \multirow{2}{*}{$\begin{array}{c}\text { Specific } \\
\text { Activity } \\
{[\text { Ci/g] }}\end{array}$} & \multirow{2}{*}{$\begin{array}{l}\text { IRCP-30 } \\
\text { Inhal. } \\
\text { DCF } \\
\text { [rem/uCi] }\end{array}$} & \multirow{2}{*}{$\begin{array}{l}\text { Release } \\
\text { Fraction }\end{array}$} & \multirow{2}{*}{$\begin{array}{l}\text { IRCP-30 } \\
\text { Inges. } \\
\text { DCF } \\
\text { [rem/uCi] }\end{array}$} & \multirow{2}{*}{$\begin{array}{l}\text { Solubility } \\
\text { Fraction }\end{array}$} & \multicolumn{2}{|c|}{$\begin{array}{l}\text { Loss to Waste } \\
\text { Fraction }\end{array}$} \\
\hline & & & & & & & & & & \\
\hline TE130 & 129.91 & & $0.00 E+00$ & $0.00 E+00$ & & & & & & \\
\hline 1127 & 126.90 & & $0.00 E+00$ & $0.00 E+00$ & & & & & & \\
\hline 1129 & 128.90 & $5.01 E+14$ & 1.38E-15 & 1.75E-04 & $1.80 \mathrm{E}-01$ & 5.00E-01 & $2.80 \mathrm{E}-01$ & $1.00 E+00$ & 1.0000 & 1.0000 \\
\hline XE128 & 127.90 & & $0.00 E+00$ & $0.00 E+00$ & & & & & & \\
\hline XE129 & 128.90 & & $0.00 E+00$ & $0.00 E+00$ & & & & & & \\
\hline XE130 & 129.90 & & $0.00 E+00$ & $0.00 E+00$ & & & & & & \\
\hline XE131 & 130.91 & & $0.00 E+00$ & $0.00 E+00$ & & & & & & \\
\hline XE132 & 131.90 & & $0.00 E+00$ & $0.00 E+00$ & & & & & & \\
\hline XE134 & 133.91 & & $0.00 E+00$ & $0.00 E+00$ & & & & & & \\
\hline XE136 & 135.91 & & $0.00 E+00$ & $0.00 E+00$ & & & & & & \\
\hline CS133 & 132.91 & & $0.00 E+00$ & $0.00 E+00$ & & & & & & \\
\hline CS134 & 133.91 & $6.50 \mathrm{E}+07$ & 1.07E-08 & 1.30E+03 & 4.70E-02 & 1.00E-02 & $7.40 E-02$ & $9.50 E-01$ & 1.0000 & 1.0000 \\
\hline CS135 & 134.91 & $7.25 E+13$ & $9.56 \mathrm{E}-15$ & 1.15E-03 & $4.50 \mathrm{E}-03$ & $1.00 \mathrm{E}-02$ & 7.10E-03 & $9.50 \mathrm{E}-01$ & 1.0000 & 1.0000 \\
\hline CS137 & 136.91. & $9.50 E+08$ & $7.30 \mathrm{E}-10$ & $8.68 E+01$ & $3.20 \mathrm{E}-02$ & $1.00 \mathrm{E}-02$ & $5.00 \mathrm{E}-02$ & $9.50 \mathrm{E}-01$ & 1.0000 & .1 .0000 \\
\hline BA134 & 133.90 & & $0.00 E+00$ & $0.00 E+00$ & & & & & & \\
\hline BA136 & 135.90 & & $0.00 E+00$ & $0.00 E+00$ & & & & & & \\
\hline BA137 & 136.91 & & $0.00 E+00$ & $0.00 E+00$ & & & & & & \\
\hline BA137M & 136.91 & $1.53 E+02$ & 4.53E-03 & $5.39 E+08$ & & & & & & \\
\hline BA138 & 137.91 & & $0.00 E+00$ & $0.00 E+00$ & & & & & & \\
\hline LA139 & 138.91 & & $0.00 E+00$ & $0.00 E+00$ & & & & & & \\
\hline CE140 & 139.91 & & $0.00 E+00$ & $0.00 E+00$ & & & & & & \\
\hline CE142 & 141.91 & $3.31 E+18$ & $2.09 E-19$ & $2.40 E-08$ & & & & & & \\
\hline CE144 & 143.91 & $2.46 E+07$ & $2.82 E-08$ & $3.19 E+03$ & 3.50E-01 & 1.00E-03 & $2.00 \mathrm{E}-02$ & $1.00 \mathrm{E}-04$ & 1.0000 & 1.0000 \\
\hline PR141 & 140.91 & & $0.00 E+00$ & $0.00 E+00$ & & & & & & \\
\hline PR144 & 143.91 & $1.04 E+03$ & $6.69 E-04$ & $7.56 \mathrm{E}+07$ & & & & & & \\
\hline PR144M & 143.91 & $4.32 E+02$ & $1.60 \mathrm{E}-03$ & $1.81 E+08$ & & & & & & \\
\hline ND142 & 141.91 & & $0.00 E+00$ & $0.00 E+00$ & & & & & & \\
\hline ND143 & 142.91 & & $0.00 E+00$ & $0.00 E+00$ & & & & & & \\
\hline ND144 & 143.91 & $6.62 E+22$ & $1.05 E-23$ & 1.18E-12 & $0.00 E+00$ & $1.00 \mathrm{E}-03$ & $0.00 E+00$ & $1.00 E-04$ & 1.0000 & 1.0000 \\
\hline ND145 & 144.91 & & $0.00 E+00$ & $0.00 E+00$ & & & & & & \\
\hline ND146 & 145.91 & & $0.00 E+00$ & $0.00 E+00$ & & & & & & \\
\hline ND148 & 147.92 & & $0.00 E+00$ & $0.00 E+00$ & & & & & & \\
\hline ND150 & 149.92 & & $0.00 E+00$ & $0.00 E+00$ & & & & & & \\
\hline PM147 & 146.91 & $8.28 E+07$ & 8.37E-09 & $9.28 E+02$ & $3.40 \mathrm{E}-02$ & $1.00 E-03$ & $9.50 E-04$ & $1.00 E-04$ & 1.0000 & 1.0000 \\
\hline PM148 & 147.92 & $4.64 E+05$ & $1.49 E-06$ & $1.64 E+05$ & $1.00 \mathrm{E}-02$ & $1.00 \mathrm{E}-03$ & $9.50 \mathrm{E}-03$ & $1.00 \mathrm{E}-04$ & 1.0000 & 1.0000 \\
\hline PM148M & 147.92 & $3.57 E+06$ & $1.94 E-07$ & $2.14 E+04$ & $1.70 \mathrm{E}-02$ & $1.00 \mathrm{E}-03$ & $7.00 \mathrm{E}-03$ & $1.00 E-04$ & 1.0000 & 1.0000 \\
\hline SM147 & 146.91 & $3.37 E+18$ & $2.05 E-19$ & $2.28 E-08$ & $7.10 E+01$ & $1.00 \mathrm{E}-03$ & $1.80 E-01$ & $3.50 E-04$ & 1.0000 & 1.0000 \\
\hline SM148 & 147.91 & $2.52 E+23$ & $2.75 E-24$ & $3.02 E-13$ & & & & & & \\
\hline SM149 & 148.92 & $3.15 E+23$ & $2.20 E-24$ & $2.40 E-13$ & - & & & & & \\
\hline SM150 & 149.92 & & $0.00 E+00$ & $0.00 E+00$ & & & & & & \\
\hline SM151 & 150.92 & $2.93 E+09$ & 2.36E-10 & 2.55E+01 & $2.90 \mathrm{E}-02$ & 1.00E-03 & $3.40 E-04$ & $3.50 E-04$ & 1.0000 & 1.0000 \\
\hline SM152 & 151.92 & & $0.00 E+00$ & $0.00 E+00$ & & & & & & \\
\hline SM154 & 153.92 & & $0.00 E+00$ & $0.00 E+00$ & & & & & & \\
\hline EU151 & 150.92 & & $0.00 E+00$ & $0.00 E+00$ & & & & & & \\
\hline EU152 & 151.92 & 4.10E+08 & 1.69E-09 & $1.81 E+02$ & 2.20E-01 & $1.00 \mathrm{E}-03$ & $6.00 E-03$ & 1.30E-04 & 1.0000 & 1.0000 \\
\hline EU153 & 152.92 & & $0.00 E+00$ & $0.00 E+00$ & & & & & & \\
\hline EU154 & 153.92 & $2.71 E+08$ & $2.56 \mathrm{E}-09$ & $2.70 E+02$ & $2.60 \mathrm{E}-01$ & $1.00 \mathrm{E}-03$ & $9.10 \mathrm{E}-03$ & $1.30 E-04$ & 1.0000 & 1.0000 \\
\hline EU155 & 154.92 & $1.51 E+08$ & $4.58 \mathrm{E}-09$ & $4.81 E+02$ & $3.90 \mathrm{E}-02$ & $1.00 \mathrm{E}-03$ & 1.30E-03 & $1.30 E-04$ & 1.0000 & 1.0000 \\
\hline
\end{tabular}


WSRC-TR-94-0456

Revision 0

Page 17 of 56

\begin{tabular}{|c|c|c|c|c|c|c|c|c|c|c|}
\hline \multirow[t]{2}{*}{ Nuclide } & \multirow[t]{2}{*}{$\begin{array}{l}\text { Atomic } \\
\text { Weight }\end{array}$} & \multirow{2}{*}{$\begin{array}{l}\text { Half Life } \\
\text { [s] }\end{array}$} & \multirow[t]{2}{*}{ Lambda } & \multirow{2}{*}{$\begin{array}{c}\text { Specific } \\
\text { Activity } \\
{[\mathrm{Ci} / \mathrm{g}]}\end{array}$} & \multirow{2}{*}{$\begin{array}{l}\text { IRCP-30 } \\
\text { Inhal. } \\
\text { DCF } \\
\text { [rem/uCi] }\end{array}$} & \multirow{2}{*}{$\begin{array}{l}\text { Release } \\
\text { Fraction }\end{array}$} & \multirow{2}{*}{$\begin{array}{l}\text { IRCP-30 } \\
\text { Inges. } \\
\text { DCF } \\
\text { [rem/uCi] }\end{array}$} & \multirow{2}{*}{$\begin{array}{l}\text { Solubility } \\
\text { Fraction }\end{array}$} & \multicolumn{2}{|c|}{$\begin{array}{c}\text { Loss to Waste } \\
\text { Fraction }\end{array}$} \\
\hline & & & & & & & & & $H M$ & PUREX \\
\hline GD152 & 1.92 & $3.47 E+21$ & 2.00E-22 & $2.14 \mathrm{E}-11$ & $2.40 \mathrm{E}+02$ & $1.00 \mathrm{E}-03$ & $1.50 \mathrm{E}-01$ & $1.00 E-04$ & 1.0000 & 1.0000 \\
\hline GD154 & 153.92 & & $0.00 E+00$ & $0.00 E+00$ & & & & & & \\
\hline GD155 & 154.92 & & $0.00 E+00$ & $0.00 E+00$ & & & & & & \\
\hline GD156 & 155.92 & & $0.00 E+00$ & $0.00 E+00$ & & & & & & \\
\hline GD157 & 156.92 & & $0.00 E+00$ & $0.00 E+00$ & & & & & & \\
\hline GD158 & 157.92 & & $E+00$ & 0.00 & & & & & & \\
\hline GD160 & 159.93 & & $E+00$ & 0,00 & & & & & & \\
\hline TB159 & 158.93 & & $0.00 \mathrm{E}+00$ & $+\infty 0$ & & & & & & \\
\hline TB160 & 159.93 & $6.25 E+06$ & 1.11E-07 & 1.13E+04 & $2.20 \mathrm{E}-02$ & 1.00E-03 & $6.40 \mathrm{E}-03$ & $1.00 \mathrm{E}-04$ & 1.0000 & 1.0000 \\
\hline DY160 & 159.93 & & $0.00 E+00$ & $0.00 \mathrm{E}+00$ & & & & & & \\
\hline DY161 & 160.93 & & $0.00 E+00$ & $0.00 E+00$ & & & & & & \\
\hline DY162 & 161.93 & & $0.00 E+00$ & $0.00 E+00$ & & & & & & \\
\hline TH232 & 232.04 & $4.43 E+17$ & $1.56 \mathrm{E}-18$ & 1.10E-07 & & & & & & \\
\hline PA233 & 233.04 & $2.33 E+06$ & 2.97E-07 & $2.08 \mathrm{E}+04$ & & & & & & \\
\hline U233 & 233.04 & $5.02 E+12$ & $1.38 E-13$ & 9.64E-03 & & & & & & \\
\hline U234 & 234.04 & $7.72 E+12$ & $8.98 E-14$ & $6.25 E-03$ & $1.30 \mathrm{E}+02$ & 1.00E-03 & 2.60E-01 & $3.00 E-04$ & 0.0015 & 0.0002 \\
\hline U235 & 235.04 & $2.22 E+16$ & $3.12 E-17$ & 2.16E-06 & $1.20 \mathrm{E}+02$ & 1.00E-03 & $2.50 E-01$ & $3.00 E-04$ & 0.0015 & 0.0002 \\
\hline U236 & 236.05 & $7.39 E+14$ & $9.38 E-16$ & 6.47E-05 & $1.20 \mathrm{E}+02$ & $1.00 E-03$ & $2.50 E-01$ & $3.00 E-04$ & 0.0015 & 0.0002 \\
\hline U237 & 237.54 & $5.83 E+05$ & 1.19E-06 & $8.14 E+04$ & & & & & & \\
\hline U238 & 238.05 & $1.41 E+17$ & $4.92 E-18$ & 3.36E-07 & $1.20 \mathrm{E}+02$ & 1.00E-03 & $2.30 E-01$ & $3.00 \mathrm{E}$ & 0.0015 & 0.0002 \\
\hline NP237 & 237.05 & $6.75 E+13$ & 1.03E-14 & 7.05E-04 & $4.90 E+02$ & $1.00 \mathrm{E}-03$ & $3.90 E+00$ & $3.00 E-04$ & 0.0350 & 0.0600 \\
\hline PU238 & 238.05 & $2.77 E+09$ & $2.50 E-10$ & $1.71 \mathrm{E}+01$ & $4.60 E+02$ & 1.00E-03 & $3.80 E+00$ & $3.00 E-04$ & 1.0000 & 0.0022 \\
\hline PU239 & 239.05 & $7.61 E+11$ & $9.11 E-13$ & $6.20 \mathrm{E}-02$ & $5.10 E+02$ & $1.00 \mathrm{E}-03$ & $4.30 E+00$ & $3.00 E-04$ & 1.0000 & 0.0022 \\
\hline PU240 & 240.05 & $2.07 E+11$ & 3.35E-12 & $2.27 \mathrm{E}-01$ & $5.10 E+02$ & $1.00 \mathrm{E}-03$ & $4.30 E+00$ & $3.00 \mathrm{E}-04$ & 1.0000 & 0.0022 \\
\hline PU241 & 241.06 & $4.64 E+08$ & $1.49 E-09$ & $1.01 \mathrm{E}+02$ & $1.00 \mathrm{E}+01$ & $1.00 \mathrm{E}-03$ & $8.60 E-02$ & $3.00 \mathrm{E}-04$ & 1.0000 & 0.0022 \\
\hline PU242 & 242.06 & $1.19 E+13$ & $5.84 E-14$ & 3.93E-03 & $4.80 E+02$ & $1.00 E-03$ & 4.10E+00 & $3.00 \mathrm{E}-04$ & 1.0000 & 0.0022 \\
\hline
\end{tabular}


WSRC-TR-94-0456

Revision 0

Page 18 of 56

Appendix B

Sludge Dose Contribution From HM Processing 
Inhalation (Volatile): Percent contribution to dose at $4 \& 20$ years

\begin{tabular}{lcccccccc} 
& \multicolumn{3}{c}{4 years } & \multicolumn{7}{c}{20 years } \\
$\begin{array}{l}\text { Fuel } \\
\text { Exposure }\end{array}$ & 20 & 20 & 40 & 40 & 20 & 20 & 40 & 40 \\
[MWD/ft] & & & & & & & & \\
Power [MW/ft] & 0.6 & 1.8 & 0.6 & 1.8 & 0.6 & 1.8 & 0.6 & 1.8 \\
\hline \hline Sr-90 & $69 \%$ & $70 \%$ & $52 \%$ & $56 \%$ & $83 \%$ & $88 \%$ & $55 \%$ & $62 \%$ \\
Ru-106 & $1 \%$ & $1 \%$ & $1 \%$ & $1 \%$ & $0 \%$ & $0 \%$ & $0 \%$ & $0 \%$ \\
Cs-137 & $0 \%$ & $0 \%$ & $0 \%$ & $0 \%$ & $0 \%$ & $0 \%$ & $0 \%$ & $0 \%$ \\
Ce-144 & $17 \%$ & $19 \%$ & $11 \%$ & $14 \%$ & $0 \%$ & $0 \%$ & $0 \%$ & $0 \%$ \\
Pm-147 & $2 \%$ & $3 \%$ & $1 \%$ & $2 \%$ & $0 \%$ & $0 \%$ & $0 \%$ & $0 \%$ \\
Pu-238 & $8 \%$ & $5 \%$ & $31 \%$ & $24 \%$ & $13 \%$ & $8 \%$ & $42 \%$ & $34 \%$ \\
Pu-239 & $1 \%$ & $1 \%$ & $1 \%$ & $1 \%$ & $2 \%$ & $2 \%$ & $1 \%$ & $1 \%$ \\
Pu-240 & $1 \%$ & $0 \%$ & $1 \%$ & $1 \%$ & $1 \%$ & $1 \%$ & $1 \%$ & $1 \%$ \\
Pu-241 & $1 \%$ & $0 \%$ & $1 \%$ & $1 \%$ & $1 \%$ & $0 \%$ & $1 \%$ & $1 \%$ \\
\hline \hline Total & $100 \%$ & $100 \%$ & $100 \%$ & $100 \%$ & $100 \%$ & $100 \%$ & $100 \%$ & $100 \%$
\end{tabular}

Inhalation (Evaporative): Isotopic percent contribution to dose at $4 \& 20$ years

\begin{tabular}{lcccccccc} 
& \multicolumn{3}{c}{4 years } & \multicolumn{6}{c}{ Mark $12 \mathrm{~A}$} & 20 years \\
$\begin{array}{l}\text { Fuel } \\
\text { Exposure }\end{array}$ & 20 & 20 & 40 & 40 & 20 & 20 & 40 & 40 \\
[MWD/ft] & & & & & & & & \\
Power[MW/ft] & 0.6 & 1.8 & 0.6 & 1.8 & 0.6 & 1.8 & 0.6 & 1.8 \\
\hline Sr-90 & $61 \%$ & $61 \%$ & $48 \%$ & $50 \%$ & $82 \%$ & $87 \%$ & $54 \%$ & $62 \%$ \\
Ru-106 & $12 \%$ & $13 \%$ & $9 \%$ & $11 \%$ & $0 \%$ & $0 \%$ & $0 \%$ & $0 \%$ \\
Cs-137 & $1 \%$ & $1 \%$ & $1 \%$ & $1 \%$ & $1 \%$ & $1 \%$ & $1 \%$ & $1 \%$ \\
Ce-144 & $15 \%$ & $16 \%$ & $10 \%$ & $13 \%$ & $0 \%$ & $0 \%$ & $0 \%$ & $0 \%$ \\
Pm-147 & $2 \%$ & $2 \%$ & $1 \%$ & $2 \%$ & $0 \%$ & $0 \%$ & $0 \%$ & $0 \%$ \\
Pu-238 & $7 \%$ & $4 \%$ & $28 \%$ & $21 \%$ & $13 \%$ & $8 \%$ & $42 \%$ & $34 \%$ \\
Pu-239 & $1 \%$ & $1 \%$ & $1 \%$ & $1 \%$ & $2 \%$ & $2 \%$ & $1 \%$ & $1 \%$ \\
Pu-240 & $1 \%$ & $0 \%$ & $1 \%$ & $1 \%$ & $1 \%$ & $1 \%$ & $1 \%$ & $1 \%$ \\
Pu-241 & $1 \%$ & $0 \%$ & $1 \%$ & $1 \%$ & $0 \%$ & $0 \%$ & $1 \%$ & $1 \%$ \\
\hline \hline Total & $100 \%$ & $100 \%$ & $100 \%$ & $100 \%$ & $100 \%$ & $100 \%$ & $100 \%$ & $100 \%$
\end{tabular}

Ingestion: Isotopic percent contribution to dose at $4 \& 20$ years

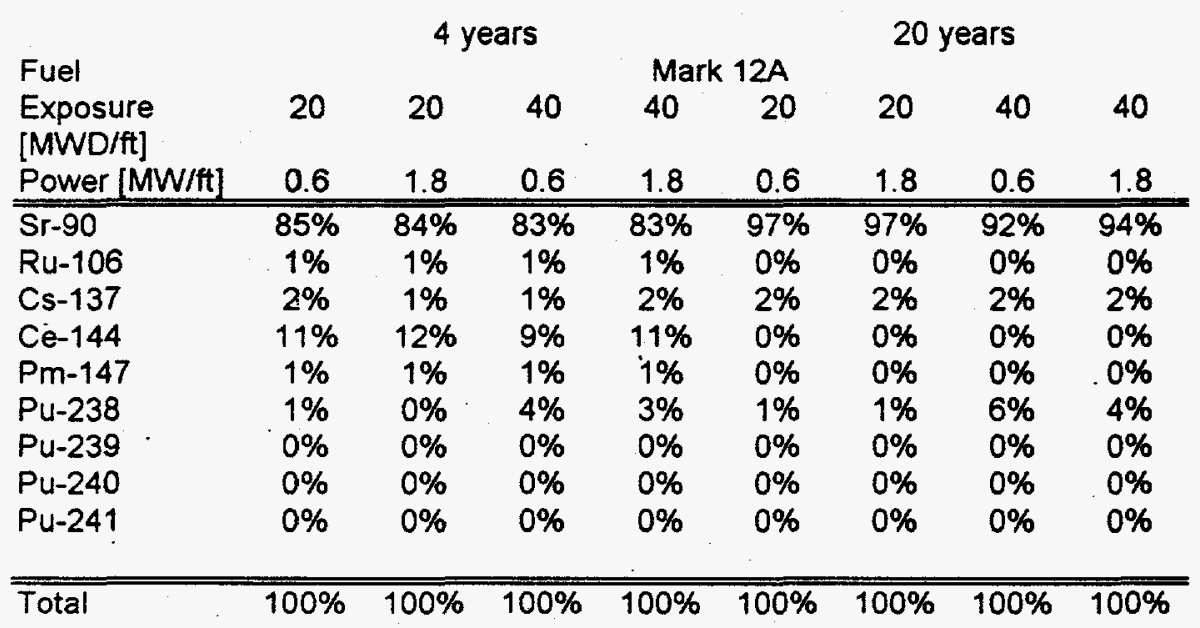


WSRC-TR-94-0456

Revision 0

Page 20 of 56

Inhalation (Volatile): Isotopic percent contribution to dose at 4 years

\begin{tabular}{|c|c|c|c|c|c|c|c|c|c|}
\hline \multirow{2}{*}{$\begin{array}{l}\text { Fuel } \\
\text { Exposure } \\
\text { [MWD/ft] } \\
\text { Power [MW/ft] }\end{array}$} & 20 & 20 & 20 & 40 & $\begin{array}{l}\text { Aark 16B } \\
.40\end{array}$ & 40 & 80 & 80 & 80 \\
\hline & 0.6 & 1.8 & 4.0 & 0.6 & 1.8 & 4.0 & 0.6 & 1.8 & 4.0 \\
\hline $\begin{array}{l}\text { Sr-90 } \\
\text { Ru-106 } \\
\text { Cs-137 } \\
\text { Ce-144 } \\
\text { Pm-147 } \\
\text { Pu-238 } \\
\text { Pu-239 } \\
\text { Pu-240 } \\
\text { Pu-241 }\end{array}$ & $\begin{array}{c}31 \% \\
0 \% \\
0 \% \\
8 \% \\
1 \% \\
53 \% \\
6 \% \\
1 \% \\
1 \%\end{array}$ & $\begin{array}{c}35 \% \\
1 \% \\
0 \% \\
9 \% \\
1 \% \\
45 \% \\
6 \% \\
1 \% \\
1 \%\end{array}$ & $\begin{array}{c}42 \% \\
1 \% \\
0 \% \\
11 \% \\
2 \% \\
35 \% \\
8 \% \\
1 \% \\
1 \%\end{array}$ & $\begin{array}{c}19 \% \\
0 \% \\
0 \% \\
4 \% \\
1 \% \\
70 \% \\
3 \% \\
1 \% \\
2 \%\end{array}$ & $\begin{array}{c}21 \% \\
0 \% \\
0 \% \\
5 \% \\
1 \% \\
66 \% \\
3 \% \\
1 \% \\
2 \%\end{array}$ & $\begin{array}{c}25 \% \\
0 \% \\
0 \% \\
7 \% \\
1 \% \\
60 \% \\
4 \% \\
1 \% \\
2 \%\end{array}$ & $\begin{array}{c}8 \% \\
0 \% \\
0 \% \\
0 \% \\
0 \% \\
88 \% \\
2 \% \\
1 \% \\
1 \%\end{array}$ & $\begin{array}{c}11 \% \\
0 \% \\
0 \% \\
3 \% \\
0 \% \\
81 \% \\
1 \% \\
1 \% \\
3 \%\end{array}$ & $\begin{array}{c}13 \% \\
0 \% \\
0 \% \\
3 \% \\
0 \% \\
78 \% \\
2 \% \\
1 \% \\
3 \%\end{array}$ \\
\hline & $3 \pi$ & $9 \%$ & $01 \%$ & \% & $9 \%$ & $99 \%$ & $00 \%$ & $00 \%$ & \\
\hline
\end{tabular}

Inhalation (Evaporative): Isotopic percent contribution to dose at 4 years

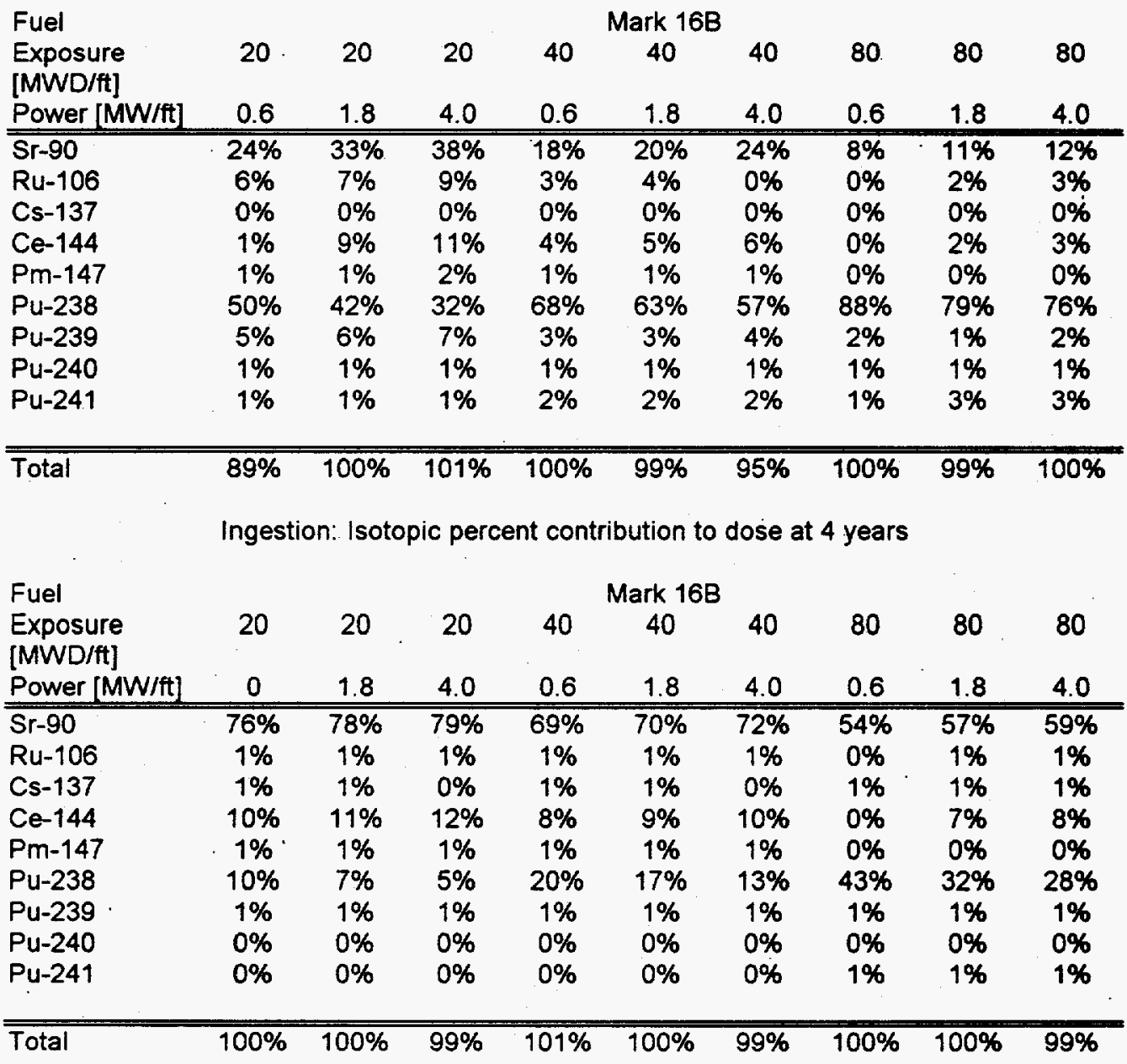


WSRC-TR-94-0456

Revision 0

Page 21 of 56

Inhalation (Volatile): Isotopic percent contribution to dose at 20 years

\begin{tabular}{lccccccccc}
$\begin{array}{l}\text { Fuel } \\
\text { Exposure }\end{array}$ & 20 & 20 & 20 & 40 & 40 & 40 & 80 & 80 & 80 \\
[MWD/ft] & & & & & & & & \\
Power [MW/ft] & 0.6 & 1.8 & 4.0 & 0.6 & 1.8 & 4.0 & 0.06 & 1.8 & 4.0 \\
\hline \hline Sr-90 & $29 \%$ & $34 \%$ & $42 \%$ & $16 \%$ & $18 \%$ & $22 \%$ & $10 \%$ & $9 \%$ & $11 \%$ \\
Ru-106 & $0 \%$ & $0 \%$ & $0 \%$ & $0 \%$ & $0 \%$ & $0 \%$ & $0 \%$ & $0 \%$ & $0 \%$ \\
Cs-137 & $0 \%$ & $0 \%$ & $0 \%$ & $0 \%$ & $0 \%$ & $0 \%$ & $0 \%$ & $0 \%$ & $0 \%$ \\
Ce-144 & $0 \%$ & $0 \%$ & $0 \%$ & $0 \%$ & $0 \%$ & $0 \%$ & $2 \%$ & $0 \%$ & $0 \%$ \\
Pm-147 & $0 \%$ & $0 \%$ & $0 \%$ & $0 \%$ & $0 \%$ & $0 \%$ & $0 \%$ & $0 \%$ & $0 \%$ \\
Pu-238 & $63 \%$ & $55 \%$ & $45 \%$ & $78 \%$ & $75 \%$ & $70 \%$ & $83 \%$ & $87 \%$ & $85 \%$ \\
Pu-239 & $5 \%$ & $9 \%$ & $11 \%$ & $4 \%$ & $4 \%$ & $5 \%$ & $1 \%$ & $2 \%$ & $2 \%$ \\
Pu-240 & $1 \%$ & $1 \%$ & $1 \%$ & $1 \%$ & $1 \%$ & $1 \%$ & $1 \%$ & $1 \%$ & $1 \%$ \\
Pu-241 & $1 \%$ & $1 \%$ & $1 \%$ & $1 \%$ & $1 \%$ & $1 \%$ & $2 \%$ & $1 \%$ & $2 \%$ \\
\hline \hline Total & $98 \%$ & $100 \%$ & $100 \%$ & $100 \%$ & $99 \%$ & $100 \%$ & $100 \%$ & $100 \%$ & $101 \%$ \\
\hline
\end{tabular}

Inhalation (Evaporative): Isotopic percent contribution to dose at 20 years

Fuel

$\begin{array}{llllllllll}\text { Exposure } & 20 & 20 & 20 & 40 & 40 & 40 & 80 & 80 & 80\end{array}$

[MWD/ft]

\begin{tabular}{lccccccccc} 
Power [MW/ft] & 0.6 & 1.8 & 4.0 & 0.6 & 1.8 & 4.0 & 0.6 & 1.8 & 4.0 \\
\hline \hline Sr-90 & $28 \%$ & $33 \%$ & $41 \%$ & $16 \%$ & $18 \%$ & $22 \%$ & $10 \%$ & $9 \%$ & $10 \%$ \\
Ru-106 & $0 \%$ & $0 \%$ & $0 \%$ & $0 \%$ & $0 \%$ & $0 \%$ & $2 \%$ & $0 \%$ & $0 \%$
\end{tabular}

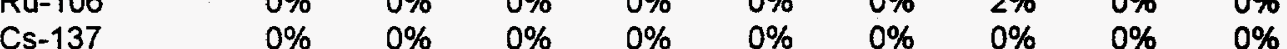

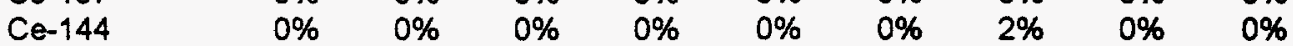

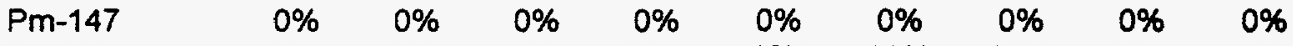

$\begin{array}{llllllllll}\text { Pu-238 } & 62 \% & 55 \% & 45 \% & 78 \% & 75 \% & 69 \% & 82 \% & 87 \% & 85 \%\end{array}$

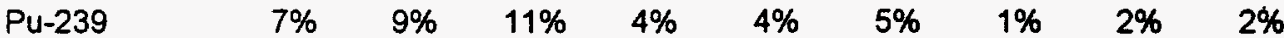

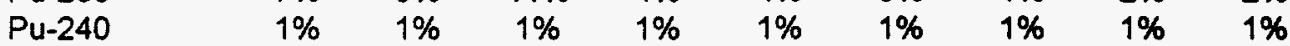

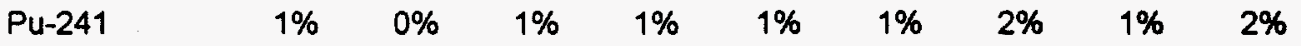

\begin{tabular}{lllllllllll}
\hline Total & $100 \%$ & $98 \%$ & $99 \%$ & $100 \%$ & $99 \%$ & $100 \%$ & $100 \%$ & $100 \%$ & $100 \%$
\end{tabular}

Ingestion; Isotopic percent contribution to dose at 20 years

\begin{tabular}{|c|c|c|c|c|c|c|c|c|c|}
\hline $\begin{array}{l}\text { Fuel } \\
\text { Exposure } \\
\text { [MWD/ft] }\end{array}$ & 20 & 20 & 20 & 40 & $\begin{array}{c}\text { Mark 16B } \\
40\end{array}$ & 40 & 80 & 80 & 80 \\
\hline Power [MW/ft] & 0.6 & 1.8 & 4.0 & 0.6 & 1.8 & 4.0 & 0.6 & 1.8 & 4.0 \\
\hline $\begin{array}{l}\text { Sr-90 } \\
\text { Ru-106 } \\
\text { Cs-137 } \\
\text { Ce-144 } \\
\text { Pm-147 } \\
\text { Pu-238 } \\
\text { Pu-239 } \\
\text { Pu-240 } \\
\text { Pu-241 }\end{array}$ & $\begin{array}{c}82 \% \\
0 \% \\
2 \% \\
0 \% \\
0 \% \\
14 \% \\
2 \% \\
0 \% \\
0 \%\end{array}$ & $\begin{array}{c}85 \% \\
0 \% \\
2 \% \\
0 \% \\
0 \% \\
11 \% \\
2 \% \\
0 \% \\
0 \%\end{array}$ & $\begin{array}{c}88 \% \\
0 \% \\
0 \% \\
0 \% \\
0 \% \\
7 \% \\
2 \% \\
0 \% \\
0 \%\end{array}$ & $\begin{array}{c}70 \% \\
0 \% \\
1 \% \\
0 \% \\
0 \% \\
26 \% \\
1 \% \\
0 \% \\
0 \%\end{array}$ & $\begin{array}{c}73 \% \\
0 \% \\
1 \% \\
0 \% \\
0 \% \\
23 \% \\
1 \% \\
0 \% \\
0 \%\end{array}$ & $\begin{array}{c}78 \% \\
1 \% \\
0 \% \\
0 \% \\
0 \% \\
19 \% \\
1 \% \\
0 \% \\
0 \%\end{array}$ & $\begin{array}{c}56 \% \\
0 \% \\
1 \% \\
5 \% \\
0 \% \\
35 \% \\
1 \% \\
0 \% \\
1 \%\end{array}$ & $\begin{array}{c}56 \% \\
0 \% \\
1 \% \\
0 \% \\
0 \% \\
41 \% \\
1 \% \\
0 \% \\
1 \%\end{array}$ & $\begin{array}{c}60 \% \\
0 \% \\
1 \% \\
0 \% \\
0 \% \\
37 \% \\
1 \% \\
0 \% \\
1 \%\end{array}$ \\
\hline
\end{tabular}


Inhalation (Volatile): Isotopic percent contribution to dose at 4 years

\begin{tabular}{lcccccccc}
$\begin{array}{l}\text { Fuel } \\
\text { Exposure }\end{array}$ & 20 & 20 & 20 & 20 & 40 & 40 & 40 & 40 \\
[MWD/ft] & & & & & & & & \\
Power [MW/ft] & 0.1 & 0.3 & 0.5 & 0.7 & 0.1 & 0.3 & 0.5 & 0.7 \\
\hline \hline Sr-90 & $28 \%$ & $31 \%$ & $34 \%$ & $36 \%$ & $17 \%$ & $19 \%$ & $19 \%$ & $21 \%$ \\
Ru-106 & $1 \%$ & $1 \%$ & $1 \%$ & $1 \%$ & $0 \%$ & $0 \%$ & $0 \%$ & $1 \%$ \\
Cs-137 & $0 \%$ & $0 \%$ & $0 \%$ & $0 \%$ & $0 \%$ & $0 \%$ & $0 \%$ & $0 \%$ \\
Ce-144 & $6 \%$ & $8 \%$ & $9 \%$ & $10 \%$ & $3 \%$ & $4 \%$ & $5 \%$ & $5 \%$ \\
Pm-147 & $1 \%$ & $1 \%$ & $1 \%$ & $1 \%$ & $1 \%$ & $1 \%$ & $1 \%$ & $1 \%$ \\
Pu-238 & $56 \%$ & $50 \%$ & $46 \%$ & $42 \%$ & $72 \%$ & $69 \%$ & $67 \%$ & $65 \%$ \\
Pu-239 & $6 \%$ & $6 \%$ & $7 \%$ & $8 \%$ & $3 \%$ & $3 \%$ & $4 \%$ & $4 \%$ \\
Pu-240 & $1 \%$ & $1 \%$ & $1 \%$ & $1 \%$ & $1 \%$ & $1 \%$ & $1 \%$ & $1 \%$ \\
Pu-241 & $1 \%$ & $1 \%$ & $1 \%$ & $1 \%$ & $3 \%$ & $3 \%$ & $3 \%$ & $3 \%$ \\
& & & & & &. & & \\
\hline \hline Total & $100 \%$ & $100 \%$ & $100 \%$ & $100 \%$ & $100 \%$ & $100 \%$ & $100 \%$ & $100 \%$
\end{tabular}

Inhalation (Volatile): Isotopic percent contribution to dose at 4 years (continued)

\begin{tabular}{lcccccccc} 
Fuel & & \multicolumn{7}{c}{ Mark 22 } \\
$\begin{array}{l}\text { Exposure } \\
\text { [MWD/ft] }\end{array}$ & 60 & 60 & 60 & 60 & 80 & 80 & 80 & 80 \\
Power [MW/ft] & 0.1 & 0.3 & 0.5 & 0.7 & 0.1 & 0.3 & 0.5 & 0.7 \\
\hline Sr-90 & $12 \%$ & $13 \%$ & $14 \%$ & $14 \%$ & $10 \%$ & $10 \%$ & $10 \%$ & $11 \%$ \\
Ru-106 & $0 \%$ & $0 \%$ & $0 \%$ & $0 \%$ & $0 \%$ & $0 \%$ & $0 \%$ & $0 \%$ \\
Cs-137 & $0 \%$ & $0 \%$ & $0 \%$ & $0 \%$ & $0 \%$ & $0 \%$ & $0 \%$ & $0 \%$ \\
Ce-144 & $2 \%$ & $3 \%$ & $3 \%$ & $4 \%$ & $1 \%$ & $2 \%$ & $2 \%$ & $3 \%$ \\
Pm-147 & $0 \%$ & $0 \%$ & $0 \%$ & $0 \%$ & $0 \%$ & $0 \%$ & $0 \%$ & $0 \%$ \\
Pu-238 & $80 \%$ & $77 \%$ & $76 \%$ & $75 \%$ & $84 \%$ & $82 \%$ & $81 \%$ & $80 \%$ \\
Pu-239 & $2 \%$ & $2 \%$ & $2 \%$ & $2 \%$ & $1 \%$ & $1 \%$ & $1 \%$ & $1 \%$ \\
Pu-240 & $1 \%$ & $1 \%$ & $1 \%$ & $1 \%$ & $1 \%$ & $1 \%$ & $1 \%$ & $1 \%$ \\
Pu-241 & $3 \%$ & $3 \%$ & $3 \%$ & $4 \%$ & $3 \%$ & $3 \%$ & $4 \%$ & $4 \%$ \\
& & & & & & & & \\
\hline \hline Total & $100 \%$ & $100 \%$ & $100 \%$ & $100 \%$ & $100 \%$ & $100 \%$ & $100 \%$ & $100 \%$
\end{tabular}

Inhalation (Evaporative): Isotopic percent contribution to dose at 4 years

Fuel

$\begin{array}{lllllllll}\text { Exposure } & 20 & 20 & 20 & 20 & 40 & 40 & 40 & 40\end{array}$

[MWD/ft]

\begin{tabular}{lcccccccc} 
Power $[\mathrm{MW} / \mathrm{ft}]$ & 0.1 & 0.3 & 0.5 & 0.7 & 0.1 & 0.3 & 0.5 & 0.7 \\
\hline \hline Sr-90 & $27 \%$ & $29 \%$ & $31 \%$ & $33 \%$ & $17 \%$ & $18 \%$ & $19 \%$ & $20 \%$
\end{tabular}

$\begin{array}{lllllllll}\text { Ru-106 } & 5 \% & 6 \% & 7 \% & 7 \% & 3 \% & 4 \% & 4 \% & 4 \%\end{array}$

$\begin{array}{lllllllll}\text { Cs-137 } & 0 \% & 0 \% & 0 \% & 0 \% & 0 \% & 0 \% & 0 \% & 0 \%\end{array}$

$\begin{array}{lllllllll}\text { Ce-144 } & 6 \% & 8 \% & 8 \% & 9 \% & 3 \% & 4 \% & 5 \% & 5 \%\end{array}$

$\begin{array}{lllllllll}\text { Pm-147 } & 1 \% & 1 \% & 1 \% & 1 \% & 1 \% & 1 \% & 1 \% & 1 \%\end{array}$

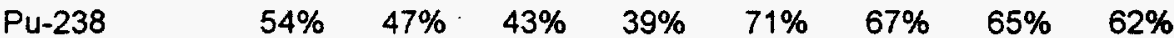

$\begin{array}{lllllllll}\text { Pu-239 } & 5 \% & 6 \% & 7 \% & 7 \% & 3 \% & 3 \% & 3 \% & 4 \%\end{array}$

$\begin{array}{lllllllll}\text { Pu-240 } & 1 \% & 1 \% & 1 \% & 1 \% & 1 \% & 1 \% & 1 \% & 1 \%\end{array}$

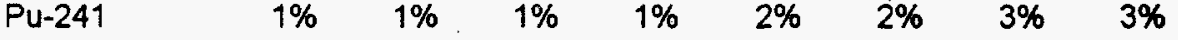

\begin{tabular}{lllllllll}
\hline \hline Total & $100 \%$ & $100 \%$ & $100 \%$ & $100 \%$ & $100 \%$ & $100 \%$ & $100 \%$ & $100 \%$
\end{tabular}


WSRC-TR-94-0456

Revision 0

Page 23 of 56

Inhalation (Evaporative): Isotopic percent contribution to dose at 4 years (continued)

\begin{tabular}{lcccccccc} 
Fuel & Mark & Mark & Mark & Mark & Mark & Mark & Mark & Mark \\
& 22 & 22 & 22 & 22 & 22 & 22 & 22 & 22 \\
Exposure & 60 & 60 & 60 & 60 & 80 & 80 & 80 & 80 \\
[MWD/ft] & & & & & & & & \\
Power [MW/ft] & 0.1 & 0.3 & 0.5 & 0.7 & 0.1 & 0.3 & 0.5 & 0.7 \\
\hline \hline Sr-90 & $12 \%$ & $13 \%$ & $13 \%$ & $14 \%$ & $9 \%$ & $10 \%$ & $10 \%$ & $11 \%$ \\
Y-90 & $0 \%$ & $0 \%$ & $0 \%$ & $0 \%$ & $0 \%$ & $0 \%$ & $0 \%$ & $0 \%$ \\
Ru-106 & $2 \%$ & $3 \%$ & $3 \%$ & $3 \%$ & $1 \%$ & $2 \%$ & $2 \%$ & $2 \%$ \\
Cs-137 & $0 \%$ & $0 \%$ & $0 \%$ & $0 \%$ & $0 \%$ & $0 \%$ & $.0 \%$ & $0 \%$ \\
Ce-144 & $2 \%$ & $3 \%$ & $3 \%$ & $4 \%$ & $1 \%$ & $2 \%$ & $2 \%$ & $3 \%$ \\
Pm-147 & $0 \%$ & $0 \%$ & $0 \%$ & $0 \%$ & $0 \%$ & $0 \%$ & $0 \%$ & $0 \%$ \\
Pu-238 & $78 \%$ & $75 \%$ & $74 \%$ & $72 \%$ & $83 \%$ & $80 \%$ & $79 \%$ & $78 \%$ \\
Pu-239 & $2 \%$ & $2 \%$ & $2 \%$ & $2 \%$ & $1 \%$ & $1 \%$ & $1 \%$ & $1 \%$ \\
Pu-240 & $1 \%$ & $1 \%$ & $1 \%$ & $1 \%$ & $1 \%$ & $1 \%$ & $1 \%$ & $1 \%$ \\
Pu-241 & $3 \%$ & $3 \%$ & $3 \%$ & $3 \%$ & $3 \%$ & $3 \%$ & $4 \%$ & $4 \%$ \\
\hline Total & $100 \%$ & $100 \%$ & $100 \%$ & $100 \%$ & $100 \%$ & $100 \%$ & $100 \%$ & $100 \%$
\end{tabular}

Ingestion: Isotopic percent contribution to dose at 4 years

\begin{tabular}{lcccccccc} 
Fuel & & \multicolumn{7}{c}{ Mark 22 } \\
$\begin{array}{l}\text { Exposure } \\
\text { [MWD/ft] }\end{array}$ & 20 & 20 & 20 & 20 & 40 & 40 & 40 & 40 \\
Power [MW/ft] & 0.1 & 0.3 & 0.5 & 0.7 & $0: 1$ & 0.3 & 0.5 & 0.7 \\
\hline \hline Sr-90 & $75 \%$ & $76 \%$ & $76 \%$ & $77 \%$ & $67 \%$ & $67 \%$ & $68 \%$ & $69 \%$ \\
Ru-106 & $1 \%$ & $1 \%$ & $1 \%$ & $1 \%$ & $1 \%$ & $1 \%$ & $1 \%$ & $1 \%$ \\
Cs-137 & $1 \%$ & $1 \%$ & $1 \%$ & $1 \%$ & $1 \%$ & $1 \%$ & $1 \%$ & $1 \%$ \\
Ce-144 & $9 \%$ & $10 \%$ & $11 \%$ & $11 \%$ & $7 \%$ & $9 \%$ & $9 \%$ & $10 \%$ \\
Pm-147 & $1 \%$ & $1 \%$ & $1 \%$ & $1 \%$ & $1 \%$ & $1 \%$ & $1 \%$ & $1 \%$ \\
Pu-238 & $12 \%$ & $9 \%$ & $8 \%$ & $7 \%$ & $22 \%$ & $19 \%$ & $18 \%$ & $17 \%$ \\
Pu-239 & $1 \%$ & $1 \%$ & $1 \%$ & $1 \%$ & $1 \%$ & $1 \%$ & $1 \%$ & $1 \%$ \\
Pu-240 & $0 \%$ & $0 \%$ & $0 \%$ & $0 \%$ & $0 \%$ & $0 \%$ & $0 \%$ & $0 \%$ \\
Pu-241 & $0 \%$ & $0 \%$ & $0 \%$ & $0 \%$ & $1 \%$ & $1 \%$ & $1 \%$ & $1 \%$ \\
& & & & & & & &
\end{tabular}

\begin{tabular}{lllllllll}
\hline \hline Total & $100 \%$ & $100 \%$ & $100 \%$ & $100 \%$ & $100 \%$ & $100 \%$ & $100 \%$ & $100 \%$
\end{tabular}

Ingestion: Isotopic percent contribution to dose at 4 years (continued)

\begin{tabular}{lcccccccc} 
Fuel & & \multicolumn{7}{c}{ Mark 22 } \\
$\begin{array}{l}\text { Exposure } \\
\text { [MWD/ft] }\end{array}$ & 60 & 60 & 60 & 60 & 80 & 80 & 80 & 80 \\
Power [MW/ft] & 0.1 & 0.3 & 0.5 & 0.7 & 0.1 & 0.3 & 0.5 & 0.7 \\
\hline \hline Sr-90 & $60 \%$ & $61 \%$ & $61 \%$ & $62 \%$ & $55 \%$ & $55 \%$ & $55 \%$ & $56 \%$ \\
Ru-106 & $0 \%$ & $1 \%$ & $1 \%$ & $1 \%$ & $0 \%$ & $1 \%$ & $1 \%$ & $1 \%$ \\
Cs-137 & $1 \%$ & $1 \%$ & $1 \%$ & $1 \%$ & $1 \%$ & $1 \%$ & $1 \%$ & $1 \%$ \\
Ce-144 & $5 \%$ & $7 \%$ & $8 \%$ & $8 \%$ & $4 \%$ & $6 \%$ & $7 \%$ & $7 \%$ \\
Pm-147 & $0 \%$ & $0 \%$ & $0 \%$ & $1 \%$ & $0 \%$ & $0 \%$ & $0 \%$ & $0 \%$ \\
Pu-238 & $30 \%$ & $28 \%$ & $26 \%$ & $25 \%$ & $37 \%$ & $34 \%$ & $33 \%$ & $32 \%$ \\
Pu-239 & $1 \%$ & $1 \%$ & $1 \%$ & $1 \%$ & $1 \%$ & $1 \%$ & $1 \%$ & $1 \%$ \\
Pu-240 & $0 \%$ & $0 \%$ & $0 \%$ & $0 \%$ & $0 \%$ & $0 \%$ & $0 \%$ & $0 \%$ \\
Pu-241 & $1 \%$ & $1 \%$ & $1 \%$ & $1 \%$ & $2 \%$ & $2 \%$ & $2 \%$ & $2 \%$ \\
& & & & & & & & \\
\hline \hline Total & $100 \%$ & $100 \%$ & $99 \%$ & $100 \%$ & $100 \%$ & $100 \%$ & $100 \%$ & $100 \%$
\end{tabular}


WSRC-TR-94-0456

Revision 0

Page 24 of 56

Inhalation (Volatile): Isotopic percent contribution to dose at 20 years

\begin{tabular}{lcccccccc} 
Fuel & & \multicolumn{7}{c}{ Mark 22 } \\
$\begin{array}{l}\text { Exposure } \\
\text { [MWD/ft] }\end{array}$ & 20 & 20 & 20 & 20 & 40 & 40 & 40 & 40 \\
Power [MW/ft] & 0.1 & 0.3 & 0.5 & 0.7 & 0.1 & 0.3 & 0.5 & 0.7 \\
\hline \hline Sr-90 & $25 \%$ & $29 \%$ & $31 \%$ & $34 \%$ & $14 \%$ & $16 \%$ & $17 \%$ & $18 \%$ \\
Ru-106 & $0 \%$ & $0 \%$ & $0 \%$ & $0 \%$ & $0 \%$ & $0 \%$ & $0 \%$ & $0 \%$ \\
Cs-137 & $0 \%$ & $0 \%$ & $0 \%$ & $0 \%$ & $0 \%$ & $0 \%$ & $0 \%$ & $0 \%$ \\
Ce-144 & $0 \%$ & $0 \%$ & $0 \%$ & $0 \%$ & $0 \%$ & $0 \%$ & $0 \%$ & $0 \%$ \\
Pm-147 & $0 \%$ & $0 \%$ & $0 \%$ & $0 \%$ & $0 \%$ & $0 \%$ & $0 \%$ & $0 \%$ \\
Pu-238 & $65 \%$ & $60 \%$ & $57 \%$ & $53 \%$ & $79 \%$ & $77 \%$ & $76 \%$ & $74 \%$ \\
Pu-239 & $7 \%$ & $9 \%$ & $10 \%$ & $11 \%$ & $4 \%$ & $4 \%$ & $4 \%$ & $5 \%$ \\
Pu-240 & $1 \%$ & $1 \%$ & $2 \%$ & $2 \%$ & $1 \%$ & $1 \%$ & $1 \%$ & $2 \%$ \\
Pu-241 & $1 \%$ & $1 \%$ & $1 \%$ & $1 \%$ & $1 \%$ & $2 \%$ & $2 \%$ & $2 \%$ \\
& & & & & & & & \\
\hline \hline Total & $100 \%$ & $100 \%$ & $100 \%$ & $100 \%$ & $100 \%$ & $100 \%$ & $100 \%$ & $100 \%$
\end{tabular}

Inhalation (Volatile): Isotopic percent contribution to dose at 20 years (continued)

\begin{tabular}{lcccccccc} 
Fuel & \multicolumn{7}{c}{ Mark 22 } \\
$\begin{array}{l}\text { Exposure } \\
\text { [MWD/ft] }\end{array}$ & 60 & 60 & 60 & 60 & 80 & 80 & 80 & 80 \\
Power [MW/ft] & 0.1 & 0.3 & 0.5 & 0.7 & 0.1 & 0.3 & 0.5 & 0.7 \\
\hline \hline Sr-90 & $10 \%$ & $11 \%$ & $11 \%$ & $12 \%$ & $8 \%$ & $8 \%$ & $9 \%$ & $9 \%$ \\
Ru-106 & $0 \%$ & $0 \%$ & $0 \%$ & $0 \%$ & $0 \%$ & $0 \%$ & $0 \%$ & $0 \%$ \\
Cs-137 & $0 \%$ & $0 \%$ & $0 \%$ & $0 \%$ & $0 \%$ & $0 \%$ & $0 \%$ & $0 \%$ \\
Ce-144 & $0 \%$ & $0 \%$ & $0 \%$ & $0 \%$ & $0 \%$ & $0 \%$ & $0 \%$ & $0 \%$ \\
Pm-147 & $0 \%$ & $0 \%$ & $0 \%$ & $0 \%$ & $0 \%$ & $0 \%$ & $0 \%$ & $0 \%$ \\
Pu-238 & $0 \%$ & $0 \%$ & $0 \%$ & $0 \%$ & $0 \%$ & $0 \%$ & $0 \%$ & $0 \%$ \\
Pu-239 & $85 \%$ & $84 \%$ & $83 \%$ & $82 \%$ & $88 \%$ & $87 \%$ & $87 \%$ & $86 \%$ \\
Pu-240 & $2 \%$ & $2 \%$ & $3 \%$ & $3 \%$ & $2 \%$ & $2 \%$ & $2 \%$ & $2 \%$ \\
Pu-241 & $1 \%$ & $1 \%$ & $1 \%$ & $1 \%$ & $1 \%$ & $1 \%$ & $1 \%$ & $1 \%$ \\
& & & & & & & & \\
\hline \hline Total & $98 \%$ & $98 \%$ & $98 \%$ & $98 \%$ & $98 \%$ & $98 \%$ & $98 \%$ & $98 \%$
\end{tabular}

Inhalation (Evaporative): Isotopic percent contribution to dose at 20 years

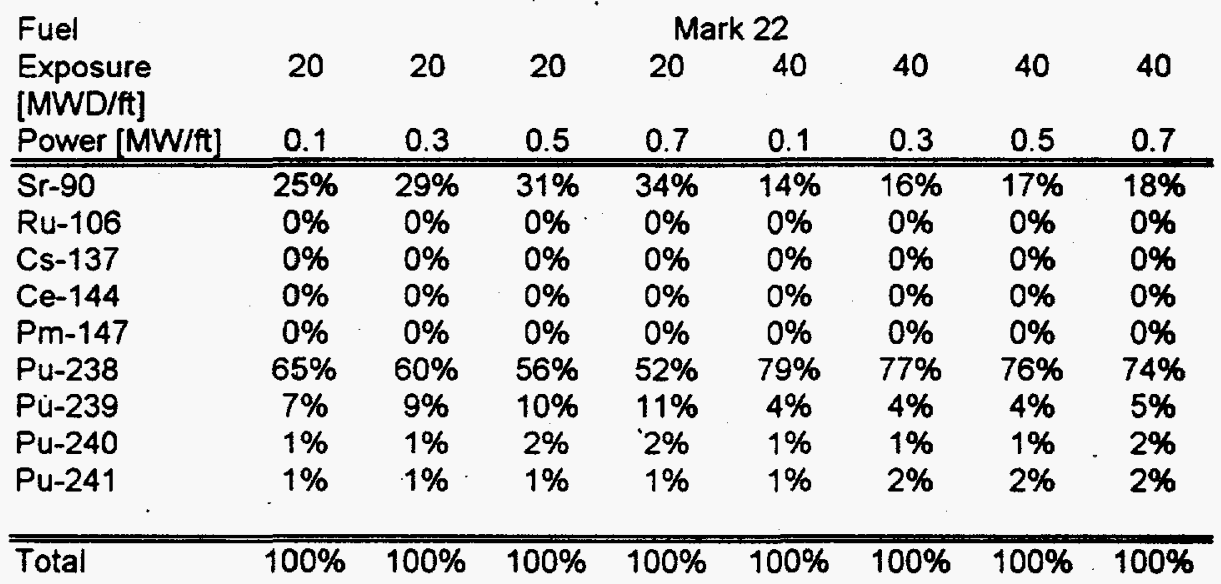


WSRC-TR-94-0456

Revision 0

Page 25 of 56

Inhalation (Evaporative): Isotopic percent contribution to dose at 20 years

(continued)

\begin{tabular}{lcccccccc}
$\begin{array}{l}\text { Fuel } \\
\text { Exposure }\end{array}$ & 60 & 60 & 60 & 60 & 80 & 80 & 80 & 80 \\
[MWD/ft] & & & & & & & & \\
Power [MW/ft] & 0.1 & 0.3 & 0.5 & 0.7 & 0.1 & 0.3 & 0.5 & 0.7 \\
\hline \hline Sr-90 & $10 \%$ & $11 \%$ & $11 \%$ & $12 \%$ & $8 \%$ & $8 \%$ & $9 \%$ & $9 \%$ \\
Ru-106 & $0 \%$ & $0 \%$ & $0 \%$ & $0 \%$ & $0 \%$ & $0 \%$ & $0 \%$ & $0 \%$ \\
Cs-137 & $0 \%$ & $0 \%$ & $0 \%$ & $0 \%$ & $0 \%$ & $0 \%$ & $0 \%$ & $0 \%$ \\
Ce-144 & $0 \%$ & $0 \%$ & $0 \%$ & $0 \%$ & $0 \%$ & $0 \%$ & $0 \%$ & $0 \%$ \\
Pm-147 & $0 \%$ & $0 \%$ & $0 \%$ & $0 \%$ & $0 \%$ & $0 \%$ & $0 \%$ & $0 \%$ \\
Pu-238 & $85 \%$ & $84 \%$ & $83 \%$ & $82 \%$ & $88 \%$ & $87 \%$ & $87 \%$ & $86 \%$ \\
Pu-239 & $2 \%$ & $2 \%$ & $3 \%$ & $3 \%$ & $2 \%$ & $2 \%$ & $2 \%$ & $2 \%$ \\
Pu-240 & $1 \%$ & $1 \%$ & $1 \%$ & $1 \%$ & $1 \%$ & $1 \%$ & $1 \%$ & $1 \%$ \\
Pu-241 & $2 \%$ & $2 \%$ & $2 \%$ & $2 \%$ & $2 \%$ & $2 \%$ & $2 \%$ & $2 \%$ \\
\hline \hline Total & $100 \%$ & $100 \%$ & $100 \%$ & $100 \%$ & $100 \%$ & $100 \%$ & $100 \%$ & $100 \%$ \\
\hline
\end{tabular}

Ingestion: Isotopic percent contribution to dose at 20 years

Fuel

$\begin{array}{lllllllll}\text { Exposure } & 20 & 20 & 20 & 20 & 40 & 40 & 40 & 40\end{array}$

[MWD/ft]

\begin{tabular}{lcccccccc} 
Power [MW/ft] & 0.1 & 0.3 & 0.5 & 0.7 & 0.1 & 0.3 & 0.5 & 0.7 \\
\hline \hline Sr-90 & $80 \%$ & $83 \%$ & $84 \%$ & $86 \%$ & $68 \%$ & $70 \%$ & $71 \%$ & $73 \%$
\end{tabular}

$\begin{array}{lllllllll}\text { Ru-106 } & 0 \% & 0 \% & 0 \% & 0 \% & 0 \% & 0 \% & 0 \% & 0 \%\end{array}$

$\begin{array}{lllllllll}\text { Cs-137 } & 2 \% & 2 \% & 2 \% & 2 \% & 1 \% & 1 \% & 1 \% & 1 \%\end{array}$

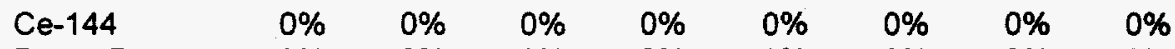

$\begin{array}{lllllllll}\text { Pm-147 } & 0 \% & 0 \% & 0 \% & 0 \% & 0 \% & 0 \% & 0 \% & 0 \%\end{array}$

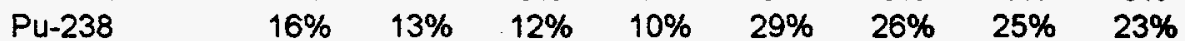

$\begin{array}{lllllllll}\text { Pu-239 } & 2 \% & 2 \% & 2 \% & 2 \% & 1 \% & 1 \% & 2 \% & 2 \%\end{array}$

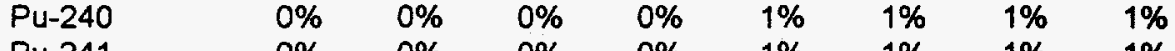

$\begin{array}{lllllllll}\text { Pu-241 } & 0 \% & 0 \% & 0 \% & 0 \% & 1 \% & 1 \% & 1 \% & 1 \%\end{array}$

\begin{tabular}{lllllllll}
\hline Total & $100 \%$ & $100 \%$ & $100 \%$ & $100 \%$ & $100 \%$ & $100 \%$ & $100 \%$ & $100 \%$
\end{tabular}

Ingestion: Isotopic percent contribution to dose at 20 years (continued)

Fuel . Mark 22

\begin{tabular}{lcccccccc} 
Exposure & 60 & 60 & 60 & 60 & 80 & 80 & 80 & 80 \\
[MWD/ft] & & & & & & & & \\
Power [MW/ft] & 0.1 & 0.3 & 0.5 & 0.7 & 0.1 & 0.3 & 0.5 & 0.7 \\
\hline \hline Sr-90 & $58 \%$ & $60 \%$ & $62 \%$ & $63 \%$ & $52 \%$ & $53 \%$ & $54 \%$ & $56 \%$ \\
Ru-106 & $0 \%$ & $0 \%$ & $0 \%$ & $0 \%$ & $0 \%$ & $0 \%$ & $0 \%$ & $0 \%$ \\
Cs-137 & $1 \%$ & $1 \%$ & $1 \%$ & $1 \%$ & $1 \%$ & $1 \%$ & $1 \%$ & $1 \%$ \\
Ce-144 & $0 \%$ & $0 \%$ & $0 \%$ & $0 \%$ & $0 \%$ & $0 \%$ & $0 \%$ & $0 \%$ \\
Pm-147 & $0 \%$ & $0 \%$ & $0 \%$ & $0 \%$ & $0 \%$ & $0 \%$ & $0 \%$ & $0 \%$ \\
Pu-238 & $38 \%$ & $36 \%$ & $35 \%$ & $33 \%$ & $45 \%$ & $43 \%$ & $43 \%$ & $41 \%$ \\
Pu-239 & $1 \%$ & $1 \%$ & $1 \%$ & $1 \%$ & $1 \%$ & $1 \%$ & $1 \%$ & $1 \%$ \\
Pu-240 & $1 \%$ & $1 \%$ & $1 \%$ & $1 \%$ & $1 \%$ & $1 \%$ & $1 \%$ & $1 \%$ \\
Pu-241 & $1 \%$ & $1 \%$ & $1 \%$ & $1 \%$ & $1 \%$ & $1 \%$ & $1 \%$ & $1 \%$ \\
& & & & & & & & \\
\hline \hline Total & $100 \%$ & $100 \%$ & $100 \%$ & $100 \%$ & $100 \%$ & $100 \%$ & $100 \%$ & $100 \%$
\end{tabular}


WSRC-TR-94-0456

Revision 0

Page 26 of 56

Inhalation (Volatile): Isotopic percent contribution to dose at 4 years

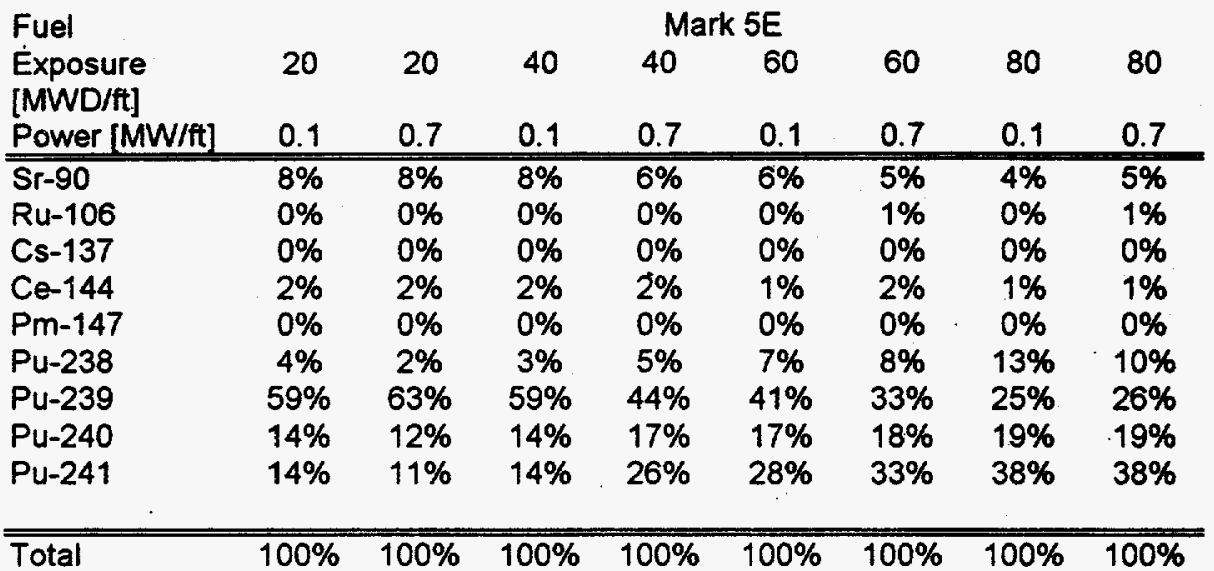

Inhalation (Evaporative): Isotopic percent contribution to dose at 4 years

\begin{tabular}{lcccccccc}
$\begin{array}{l}\text { Fuel } \\
\text { Exposure }\end{array}$ & 20 & 20 & 40 & 40 & 60 & 60 & 80 & 80 \\
$\begin{array}{l}\text { [MWD/ft] } \\
\text { Power [MW/ft] }\end{array}$ & 0.1 & 0.7 & 0.1 & 0.7 & 0.1 & 0.7 & 0.1 & 0.7 \\
\hline \hline Sr-90 & $8 \%$ & $8 \%$ & $8 \%$ & $6 \%$ & $6 \%$ & $5 \%$ & $4 \%$ & $4 \%$ \\
Ru-106 & $4 \%$ & $4 \%$ & $4 \%$ & $5 \%$ & $4 \%$ & $5 \%$ & $3 \%$ & $5 \%$ \\
Cs-137 & $0 \%$ & $0 \%$ & $0 \%$ & $0 \%$ & $0 \%$ & $0 \%$ & $0 \%$ & $0 \%$ \\
Ce-144 & $2 \%$ & $2 \%$ & $2 \%$ & $2 \%$ & $1 \%$ & $1 \%$ & $1 \%$ & $1 \%$ \\
Pm-147 & $0 \%$ & $0 \%$ & $0 \%$ & $0 \%$ & $0 \%$ & $0 \%$ & $0 \%$ & $0 \%$ \\
Pu-238 & $3 \%$ & $2 \%$ & $3 \%$ & $5 \%$ & $7 \%$ & $7 \%$ & $12 \%$ & $10 \%$ \\
Pu-239 & $56 \%$ & $61 \%$ & $56 \%$ & $42 \%$ & $39 \%$ & $31 \%$ & $24 \%$ & $25 \%$ \\
Pu-240 & $13 \%$ & $11 \%$ & $13 \%$ & $16 \%$ & $17 \%$ & $18 \%$ & $19 \%$ & $18 \%$ \\
Pu-241 & $13 \%$ & $11 \%$ & $13 \%$ & $25 \%$ & $27 \%$ & $32 \%$ & $37 \%$ & $36 \%$ \\
& & & & & & & & \\
\hline \hline Total & $100 \%$ & $100 \%$ & $100 \%$ & $100 \%$ & $100 \%$ & $100 \%$ & $100 \%$ & $100 \%$
\end{tabular}

Ingestion: Isotopic percent contribution to dose at 4 years

\begin{tabular}{lcccccccc} 
Fuel & \multicolumn{8}{c}{ Mark $5 \mathrm{E}$} \\
$\begin{array}{l}\text { Exposure } \\
\text { [MWD/ft] }\end{array}$ & 20 & 20 & 40 & 40 & 60 & 60 & 80 & 80 \\
Power [MW/ft] & 0.1 & 0.7 & 0.1 & 0.7 & 0.1 & 0.7 & 0.1 & 0.7 \\
\hline Sr-90 & $48 \%$ & $49 \%$ & $48 \%$ & $43 \%$ & $42 \%$ & $39 \%$ & $35 \%$ & $35 \%$ \\
Ru-106 & $1 \%$ & $1 \%$ & $1 \%$ & $1 \%$ & $1 \%$ & $2 \%$ & $1 \%$ & $2 \%$ \\
Cs-137 & $1 \%$ & $1 \%$ & $1 \%$ & $1 \%$ & $1 \%$ & $1 \%$ & $1 \%$ & $1 \%$ \\
Ce-144 & $6 \%$ & $7 \%$ & $6 \%$ & $7 \%$ & $4 \%$ & $6 \%$ & $3 \%$ & $5 \%$ \\
Pm-147 & $1 \%$ & $1 \%$ & $0 \%$ & $0 \%$ & $0 \%$ & $0 \%$ & $0 \%$ & $0 \%$ \\
Pu-238 & $2 \%$ & $1 \%$ & $2 \%$ & $3 \%$ & $4 \%$ & $4 \%$ & $8 \%$ & $6 \%$ \\
Pu-239 & $28 \%$ & $29 \%$ & $28 \%$ & $23 \%$ & $22 \%$ & $19 \%$ & $16 \%$ & $16 \%$ \\
Pu-240 & $7 \%$ & $6 \%$ & $7 \%$ & $9 \%$ & $9 \%$ & $10 \%$ & $12 \%$ & $11 \%$ \\
Pu-241 & $7 \%$ & $5 \%$ & $7 \%$ & $14 \%$ & $15 \%$ & $19 \%$ & $24 \%$ & $23 \%$ \\
\hline Total & $100 \%$ & $100 \%$ & $100 \%$ & $100 \%$ & $100 \%$ & $100 \%$ & $100 \%$ & $100 \%$ \\
\hline
\end{tabular}


WSRC-TR-94-0456

Revision 0

Page 27 of 56

Inhalation (Volatile): Isotopic percent contribution to dose at 20 years

\begin{tabular}{lcccccccc} 
Fuel & & \multicolumn{7}{c}{ Mark 5E } \\
$\begin{array}{l}\text { Exposure } \\
\text { [MWD/ft] }\end{array}$ & 20 & 20 & 40 & 40 & 60 & 60 & 80 & 80 \\
Power [MW/ft] & 0.1 & 0.7 & 0.1 & 0.7 & 0.1 & 0.7 & 0.1 & 0.7 \\
\hline \hline Sr-90 & $6 \%$ & $6 \%$ & $6 \%$ & $5 \%$ & $5 \%$ & $5 \%$ & $4 \%$ & $4 \%$ \\
Ru-106 & $0 \%$ & $0 \%$ & $0 \%$ & $0 \%$ & $0 \%$ & $0 \%$ & $0 \%$ & $0 \%$ \\
Cs-137 & $0 \%$ & $0 \%$ & $0 \%$ & $0 \%$ & $0 \%$ & $0 \%$ & $0 \%$ & $0 \%$ \\
Ce-144 & $0 \%$ & $0 \%$ & $0 \%$ & $0 \%$ & $0 \%$ & $0 \%$ & $0 \%$ & $0 \%$ \\
Pm-147 & $0 \%$ & $0 \%$ & $0 \%$ & $0 \%$ & $0 \%$ & $0 \%$ & $0 \%$ & $0 \%$ \\
Pu-238 & $4 \%$ & $2 \%$ & $4 \%$ & $5 \%$ & $7 \%$ & $9 \%$ & $15 \%$ & $12 \%$ \\
Pu-239 & $67 \%$ & $72 \%$ & $67 \%$ & $54 \%$ & $50 \%$ & $43 \%$ & $33 \%$ & $35 \%$ \\
Pu-240 & $16 \%$ & $14 \%$ & $16 \%$ & $20 \%$ & $21 \%$ & $24 \%$ & $25 \%$ & $25 \%$ \\
Pu-241. & $7 \%$ & $6 \%$ & $7 \%$ & $15 \%$ & $16 \%$ & $20 \%$ & $23 \%$ & $24 \%$ \\
& & & & & & & & \\
\hline \hline Total & $100 \%$ & $100 \%$ & $100 \%$ & $100 \%$ & $100 \%$ & $100 \%$ & $100 \%$ & $100 \%$
\end{tabular}

Inhalation (Evaporative): Isotopic percent contribution to dose at 20 years

\begin{tabular}{lcccccccc} 
Fuel & \multicolumn{7}{c}{ Mark 5E } \\
$\begin{array}{l}\text { Exposure } \\
\text { [MWD/ft] }\end{array}$ & 20 & 20 & 40 & 40 & 60 & 60 & 80 & 80 \\
Power [MW/ft] & 0.1 & 0.7 & 0.1 & 0.7 & 0.1 & 0.7 & 0.1 & 0.7 \\
\hline \hline Sr-90 & $6 \%$ & $6 \%$ & $6 \%$ & $5 \%$ & $5 \%$ & $5 \%$ & $4 \%$ & $4 \%$ \\
Ru-106 & $0 \%$ & $0 \%$ & $0 \%$ & $0 \%$ & $0 \%$ & $0 \%$ & $0 \%$ & $0 \%$ \\
Cs-137 & $0 \%$ & $0 \%$ & $0 \%$ & $0 \%$ & $0 \%$ & $0 \%$ & $0 \%$ & $0 \%$ \\
Ce-144 & $0 \%$ & $0 \%$ & $0 \%$ & $0 \%$ & $0 \%$ & $0 \%$ & $0 \%$ & $0 \%$ \\
Pm-147 & $0 \%$ & $0 \%$ & $0 \%$ & $0 \%$ & $0 \%$ & $0 \%$ & $0 \%$ & $0 \%$ \\
Pu-238 & $4 \%$ & $2 \%$ & $4 \%$ & $5 \%$ & $7 \%$ & $9 \%$ & $15 \%$ & $12 \%$ \\
Pu-239 & $67 \%$ & $72 \%$ & $67 \%$ & $54 \%$ & $50 \%$ & $43 \%$ & $33 \%$ & $35 \%$ \\
Pu-240 & $16 \%$ & $14 \%$ & $16 \%$ & $20 \%$ & $21 \%$ & $24 \%$ & $25 \%$ & $25 \%$ \\
Pu-241 & $7 \%$ & $6 \%$ & $7 \%$ & $15 \%$ & $16 \%$ & $20 \%$ & $23 \%$ & $24 \%$ \\
& & & & & & & & \\
\hline \hline Total & $100 \%$ & $100 \%$ & $100 \%$ & $100 \%$ & $100 \%$ & $100 \%$ & $100 \%$ & $100 \%$
\end{tabular}

Ingestion: Isotopic percent contribution to dose at 20 years

\begin{tabular}{lcccccccc} 
Fuel & & \multicolumn{7}{c}{ Mark 5E } \\
$\begin{array}{l}\text { Exposure } \\
\text { [MWD/ft] }\end{array}$ & 20 & 20 & 40 & 40 & 60 & 60 & 80 & 80 \\
Power [MW/ft] & 0.1 & 0.7 & 0.1 & 0.7 & 0.1 & 0.7 & 0.1 & 0.7 \\
\hline \hline Sr-90 & $45 \%$ & $46 \%$ & $6 \%$ & $41 \%$ & $40 \%$ & $38 \%$ & $34 \%$ & $35 \%$ \\
Ru-106 & $0 \%$ & $0 \%$ & $0 \%$ & $0 \%$ & $0 \%$ & $0 \%$ & $0 \%$ & $0 \%$ \\
Cs-137 & $1 \%$ & $1 \%$ & $0 \%$ & $1 \%$ & $1 \%$ & $1 \%$ & $1 \%$ & $1 \%$ \\
Ce-144 & $0 \%$ & $0 \%$ & $0 \%$ & $0 \%$ & $0 \%$ & $0 \%$ & $0 \%$ & $0 \%$ \\
Pm-147 & $0 \%$ & $0 \%$ & $0 \%$ & $0 \%$ & $0 \%$ & $0 \%$ & $0 \%$ & $0 \%$ \\
Pu-238 & $2 \%$ & $1 \%$ & $4 \%$ & $3 \%$ & $4 \%$ & $6 \%$ & $10 \%$ & $8 \%$ \\
Pu-239 & $39 \%$ & $41 \%$ & $67 \%$ & $33 \%$ & $31 \%$ & $27 \%$ & $22 \%$ & $23 \%$ \\
Pu-240 & $9 \%$ & $8 \%$ & $16 \%$ & $12 \%$ & $13 \%$ & $15 \%$ & $17 \%$ & $17 \%$ \\
Pu-241 & $4 \%$ & $3 \%$ & $7 \%$ & $9 \%$ & $10 \%$ & $13 \%$ & $16 \%$ & $16 \%$ \\
\hline \hline Total & $100 \%$ & $100 \%$ & $100 \%$ & $100 \%$ & $100 \%$ & $100 \%$ & $100 \%$ & $100 \%$ \\
\hline
\end{tabular}


WSRC-TR-94-0456

Revision 0

Page 28 of 56

Appendix C

Sludge Dose Contribution From PUREX Processing 
WSRC-TR-94-0456

Revision 0

Page 29 of 56

Inhalation (Volatile): Isotopic percent contribution to dose at 4 years

\begin{tabular}{lccccccccc} 
Target & & \multicolumn{8}{c}{ Mark 31A } \\
$\begin{array}{lcccc}\text { Exposure } \\
\text { [MWD/ft] }\end{array}$ & 2.5 & 2.5 & 2.5 & 4.0 & 4.0 & 4.0 & 6.75 & 6.75 & 6.75 \\
Power [MW/ft] & 0.6 & 1.8 & 4.0 & 0.6 & 1.8 & 4.0 & 0.6 & 1.8 & 4.0 \\
\hline Sr-90 & $68 \%$ & $68 \%$ & $68 \%$ & $67 \%$ & $67 \%$ & $67 \%$ & $66 \%$ & $65 \%$ & $65 \%$ \\
Ru-106 & $5 \%$ & $4 \%$ & $4 \%$ & $5 \%$ & $5 \%$ & $5 \%$ & $7 \%$ & $7 \%$ & $6 \%$ \\
Cs-137 & $0 \%$ & $0 \%$ & $0 \%$ & $0 \%$ & $0 \%$ & $0 \%$ & $0 \%$ & $0 \%$ & $0 \%$ \\
Ce-144 & $19 \%$ & $20 \%$ & $20 \%$ & $19 \%$ & $20 \%$ & $20 \%$ & $18 \%$ & $20 \%$ & $20 \%$ \\
Pm-147 & $3 \%$ & $3 \%$ & $3 \%$ & $3 \%$ & $3 \%$ & $3 \%$ & $3 \%$ & $3 \%$ & $3 \%$ \\
Pu-238 & $0 \%$ & $0 \%$ & $0 \%$ & $0 \%$ & $0 \%$ & $0 \%$ & $0 \%$ & $0 \%$ & $0 \%$ \\
Pu-239 & $5 \%$ & $5 \%$ & $5 \%$ & $4 \%$ & $4 \%$ & $4 \%$ & $4 \%$ & $4 \%$ & $4 \%$ \\
Pu-240 & $0 \%$ & $0 \%$ & $0 \%$ & $0 \%$ & $0 \%$ & $0 \%$ & $1 \%$ & $1 \%$ & $1 \%$ \\
Pu-241 & $0 \%$ & $0 \%$ & $0 \%$ & $0 \%$ & $0 \%$ & $0 \%$ & $1 \%$ & $0 \%$ & $0 \%$ \\
\hline \hline Total & $100 \%$ & $100 \%$ & $100 \%$ & $100 \%$ & $100 \%$ & $100 \%$ & $100 \%$ & $100 \%$ & $100 \%$ \\
\hline
\end{tabular}

Inhalation (Evaporative): Isotopic percent contribution to dose at 4 years

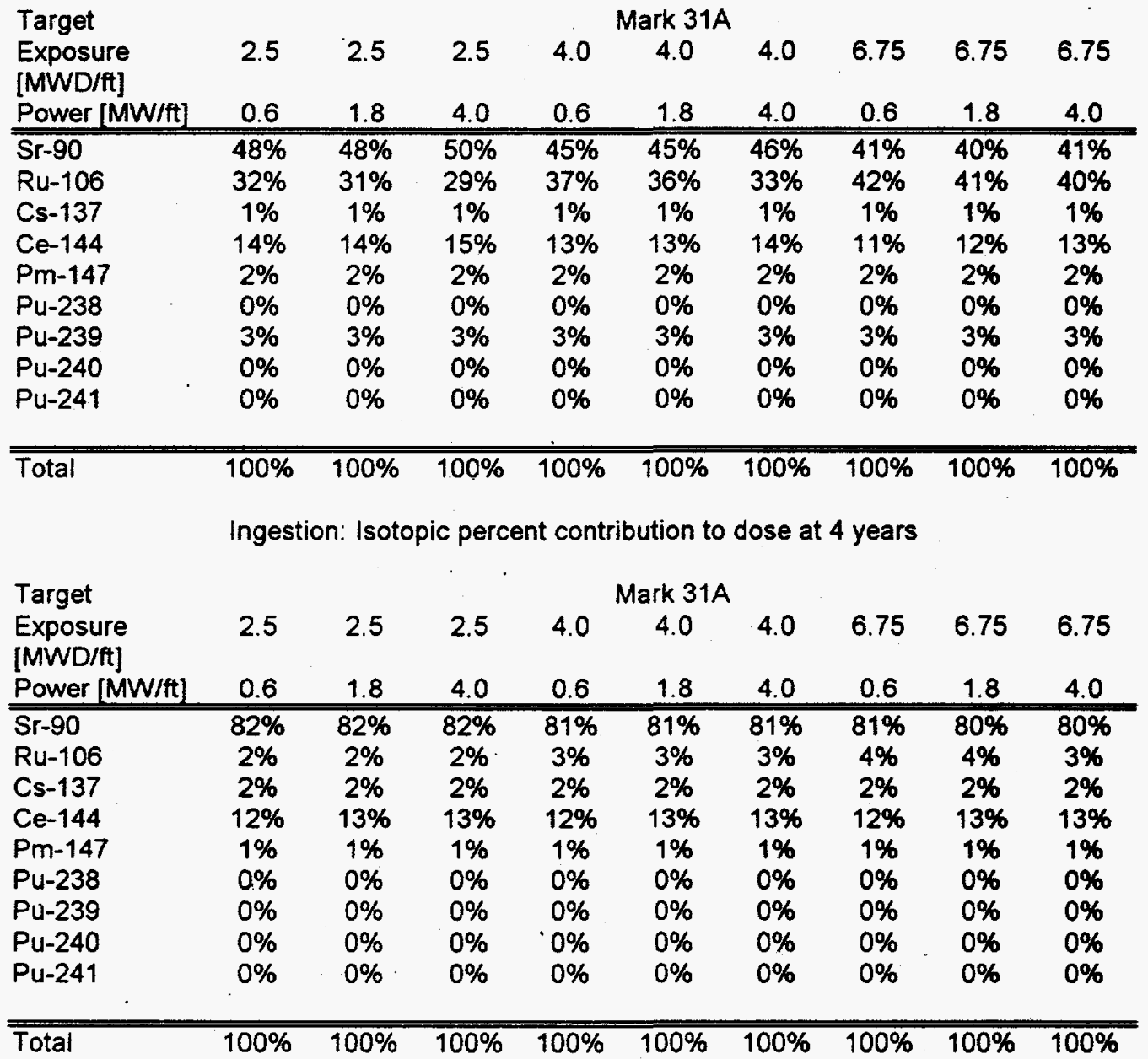


WSRC-TR-94-0456

Revision 0

Page 30 of 56

Inhalation (Volatile): Isotopic percent contribution to dose at 20 years

\begin{tabular}{lccccccccc}
$\begin{array}{l}\text { Target } \\
\text { Exposure } \\
\text { [MWD/ft] }\end{array}$ & 2.5 & 2.5 & 2.5 & 4.0 & 4.0 & 4.0 & 6.75 & 6.75 & 6.75 \\
Power [MW/ft] & 0.6 & 1.8 & 4.0 & 0.6 & 1.8 & 4.0 & 0.6 & 1.8 & 4.0 \\
\hline \hline Sr-90 & $90 \%$ & $90 \%$ & $90 \%$ & $90 \%$ & $90 \%$ & $90 \%$ & $90 \%$ & $90 \%$ & $90 \%$ \\
Ru-106 & $0 \%$ & $0 \%$ & $0 \%$ & $0 \%$ & $0 \%$ & $0 \%$ & $0 \%$ & $0 \%$ & $0 \%$ \\
Cs-137 & $0 \%$ & $0 \%$ & $0 \%$ & $0 \%$ & $0 \%$ & $0 \%$ & $0 \%$ & $0 \%$ & $0 \%$ \\
Ce-144 & $0 \%$ & $0 \%$ & $0 \%$ & $0 \%$ & $0 \%$ & $0 \%$ & $0 \%$ & $0 \%$ & $0 \%$ \\
Pm-147 & $0 \%$ & $0 \%$ & $0 \%$ & $0 \%$ & $0 \%$ & $0 \%$ & $0 \%$ & $0 \%$ & $0 \%$ \\
Pu-238 & $0 \%$ & $0 \%$ & $0 \%$ & $0 \%$ & $0 \%$ & $0 \%$ & $0 \%$ & $0 \%$ & $0 \%$ \\
Pu-239 & $9 \%$ & $9 \%$ & $9 \%$ & $9 \%$ & $9 \%$ & $9 \%$ & $8 \%$ & $8 \%$ & $8 \%$ \\
Pu-240 & $1 \%$ & $0 \%$ & $0 \%$ & $1 \%$ & $1 \%$ & $1 \%$ & $1 \%$ & $1 \%$ & $1 \%$ \\
Pu-241 & $0 \%$ & $0 \%$ & $0 \%$ & $0 \%$ & $0 \%$ & $0 \%$ & $0 \%$ & $0 \%$ & $0 \%$ \\
\hline \hline Total & $100 \%$ & $100 \%$ & $100 \%$ & $100 \%$ & $100 \%$ & $100 \%$ & $100 \%$ & $100 \%$ & $100 \%$ \\
\hline
\end{tabular}

Inhalation (Evaporative): Isotopic percent contribution to dose at 20 years

\begin{tabular}{|c|c|c|c|c|c|c|c|c|c|}
\hline \multirow{3}{*}{$\begin{array}{l}\text { Target } \\
\text { Exposure } \\
\text { [MWD/ft] } \\
\text { Power [MW/ft] }\end{array}$} & \multicolumn{9}{|c|}{ Mark 31A } \\
\hline & 2.5 & 2.5 & 2.5 & 4.0 & 4.0 & 4.0 & 6.75 & 6.75 & 6.75 \\
\hline & 0.6 & 1.8 & 4.0 & 0.6 & 1.8 & 4.0 & 0.6 & 1.8 & 4.0 \\
\hline $\begin{array}{l}\text { Sr-90 } \\
\text { Ru-106 } \\
\text { Cs-137 } \\
\text { Ce-144 } \\
\text { Pm-147 } \\
\text { Pu-238 } \\
\text { Pu-239 } \\
\text { Pu-240 } \\
\text { Pu-241 }\end{array}$ & $\begin{array}{c}89 \% \\
0 \% \\
1 \% \\
0 \% \\
0 \% \\
0 \% \\
9 \% \\
1 \% \\
0 \%\end{array}$ & $\begin{array}{c}89 \% \\
0 \% \\
1 \% \\
0 \% \\
0 \% \\
0 \% \\
9 \% \\
0 \% \\
0 \%\end{array}$ & $\begin{array}{c}89 \% \\
0 \% \\
1 \% \\
0 \% \\
0 \% \\
0 \% \\
9 \% \\
0 \% \\
0 \%\end{array}$ & $\begin{array}{c}89 \% \\
0 \% \\
1 \% \\
0 \% \\
0 \% \\
0 \% \\
9 \% \\
1 \% \\
0 \%\end{array}$ & $\begin{array}{l}89 \% \\
0 \% \\
1 \% \\
0 \% \\
0 \% \\
0 \% \\
9 \% \\
1 \% \\
0 \%\end{array}$ & $\begin{array}{c}89 \% \\
0 \% \\
1 \% \\
0 \% \\
0 \% \\
0 \% \\
9 \% \\
1 \% \\
0 \%\end{array}$ & $\begin{array}{c}88 \% \\
0 \% \\
1 \% \\
0 \% \\
0 \% \\
0 \% \\
8 \% \\
1 \% \\
0 \%\end{array}$ & $\begin{array}{l}89 \% \\
0 \% \\
1 \% \\
0 \% \\
0 \% \\
0 \% \\
8 \% \\
1 \% \\
0 \%\end{array}$ & $\begin{array}{c}89 \% \\
0 \% \\
1 \% \\
0 \% \\
0 \% \\
0 \% \\
8 \% \\
1 \% \\
0 \%\end{array}$ \\
\hline \multicolumn{10}{|c|}{ Ingestion: Isotopic percent contribution to dose at 20 years } \\
\hline $\begin{array}{l}\text { Target } \\
\text { Exposure } \\
\text { [MWD/ft] } \\
\text { Power [MW/ft] }\end{array}$ & 2.5 & 2.5 & 2.5 & 4.0 & $\begin{array}{c}\text { Mark } 31 \\
4.0\end{array}$ & $\begin{array}{l}4.0 \\
4.0\end{array}$ & $\begin{array}{l}6.75 \\
0.6\end{array}$ & $\begin{array}{c}6.75 \\
1.8\end{array}$ & 6.75 \\
\hline $\begin{array}{l}\text { Sr-90 } \\
\text { Ru-106 } \\
\text { Cs-137 } \\
\text { Ce-144 } \\
\text { Pm-147 } \\
\text { Pu-238 } \\
\text { Pu-239 } \\
\text { Pu-240 } \\
\text { Pu-241 }\end{array}$ & $\begin{array}{c}97 \% \\
0 \% \\
2 \% \\
0 \% \\
0 \% \\
0 \% \\
1 \% \\
0 \% \\
0 \%\end{array}$ & $\begin{array}{c}97 \% \\
0 \% \\
2 \% \\
0 \% \\
0 \% \\
0 \% \\
1 \% \\
0 \% \\
0 \%\end{array}$ & $\begin{array}{c}97 \% \\
0 \% \\
2 \% \\
0 \% \\
0 \% \\
0 \% \\
1 \% \\
0 \% \\
0 \%\end{array}$ & $\begin{array}{l}97 \% \\
0 \% \\
2 \% \\
0 \% \\
0 \% \\
0 \% \\
1 \% \\
0 \% \\
0 \%\end{array}$ & $\begin{array}{l}97 \% \\
0 \% \\
2 \% \\
0 \% \\
0 \% \\
0 \% \\
1 \% \\
0 \% \\
0 \%\end{array}$ & $\begin{array}{c}97 \% \\
0 \% \\
2 \% \\
0 \% \\
0 \% \\
0 \% \\
1 \% \\
0 \% \\
0 \%\end{array}$ & $\begin{array}{l}97 \% \\
0 \% \\
2 \% \\
0 \% \\
0 \% \\
0 \% \\
1 \% \\
0 \% \\
0 \%\end{array}$ & $\begin{array}{l}97 \% \\
0 \% \\
2 \% \\
0 \% \\
0 \% \\
0 \% \\
1 \% \\
0 \% \\
0 \%\end{array}$ & $\begin{array}{l}97 \% \\
0 \% \\
2 \% \\
0 \% \\
0 \% \\
0 \% \\
1 \% \\
0 \% \\
0 \%\end{array}$ \\
\hline ota & $100 \%$ & $100 \%$ & $100 \%$ & $100 \%$ & $100 \%$ & $100 \%$ & $100 \%$ & $100 \%$ & $100^{\circ}$ \\
\hline
\end{tabular}


WSRC-TR-94-0456

Revision 0

Page 31 of 56

\section{Appendix D}

Tank Inventory Radionuclide Comparisons, HM Processing 
WSRC-TR-94-0456

Revision 0

Page 32 of 56

Figure D-1

Radionuclides in Mark 22 Sludge At Various Ages

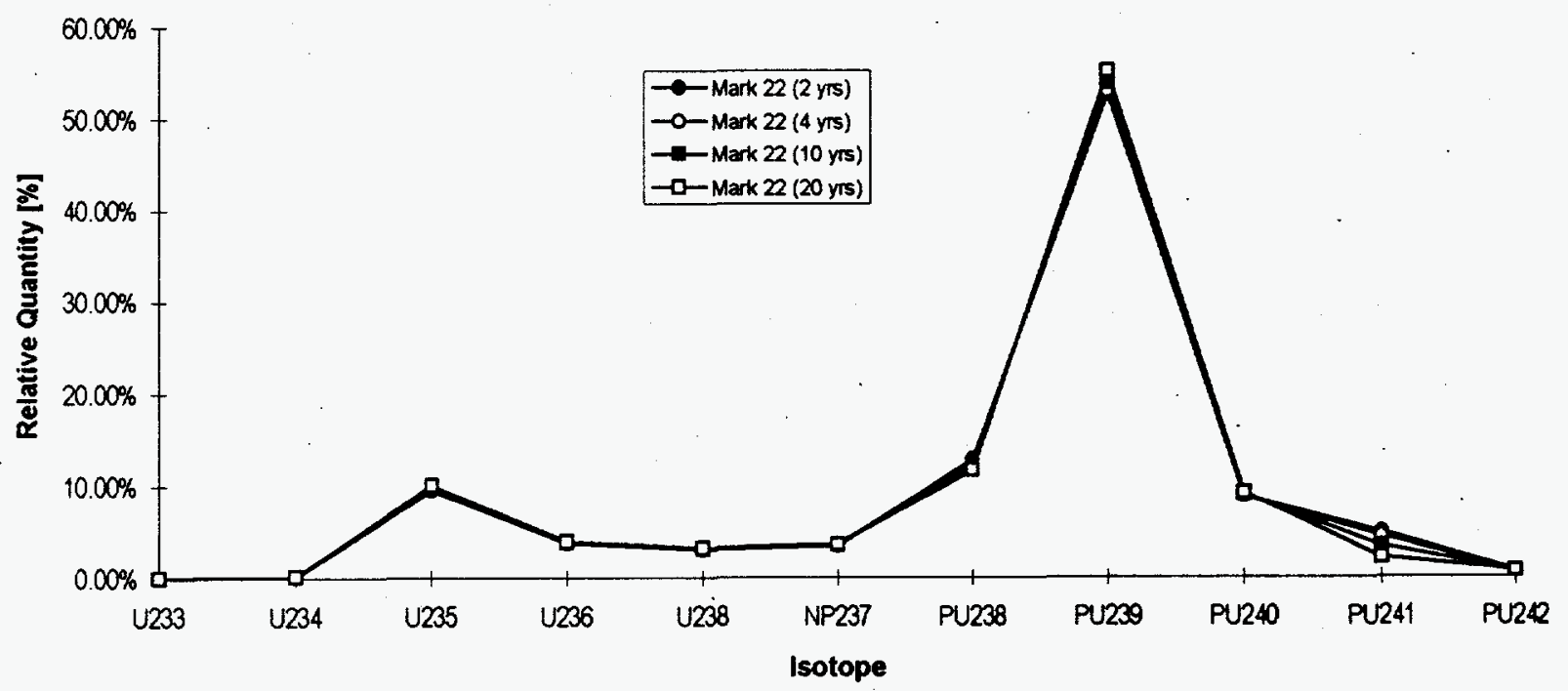


WSRC-TR-94-0456

Revision 0

Page 33 of 56

Figure D-2

Radionuclides in Mark 22 Sludge At Various Power Levels

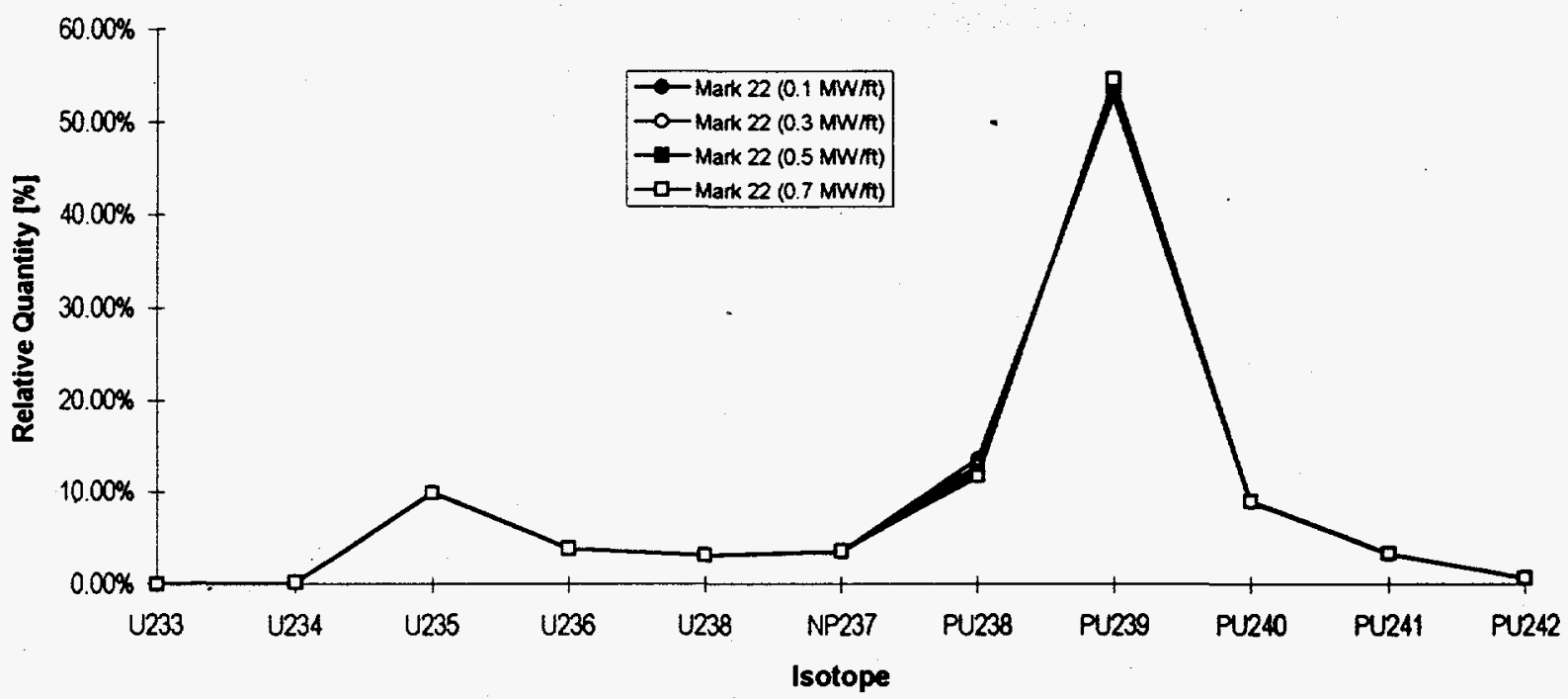


WSRC-TR-94-0456

Revision 0

Page 34 of 56

Figure D-3

Radionuclides in Mark 22 Sludge Waste At Various Exposures

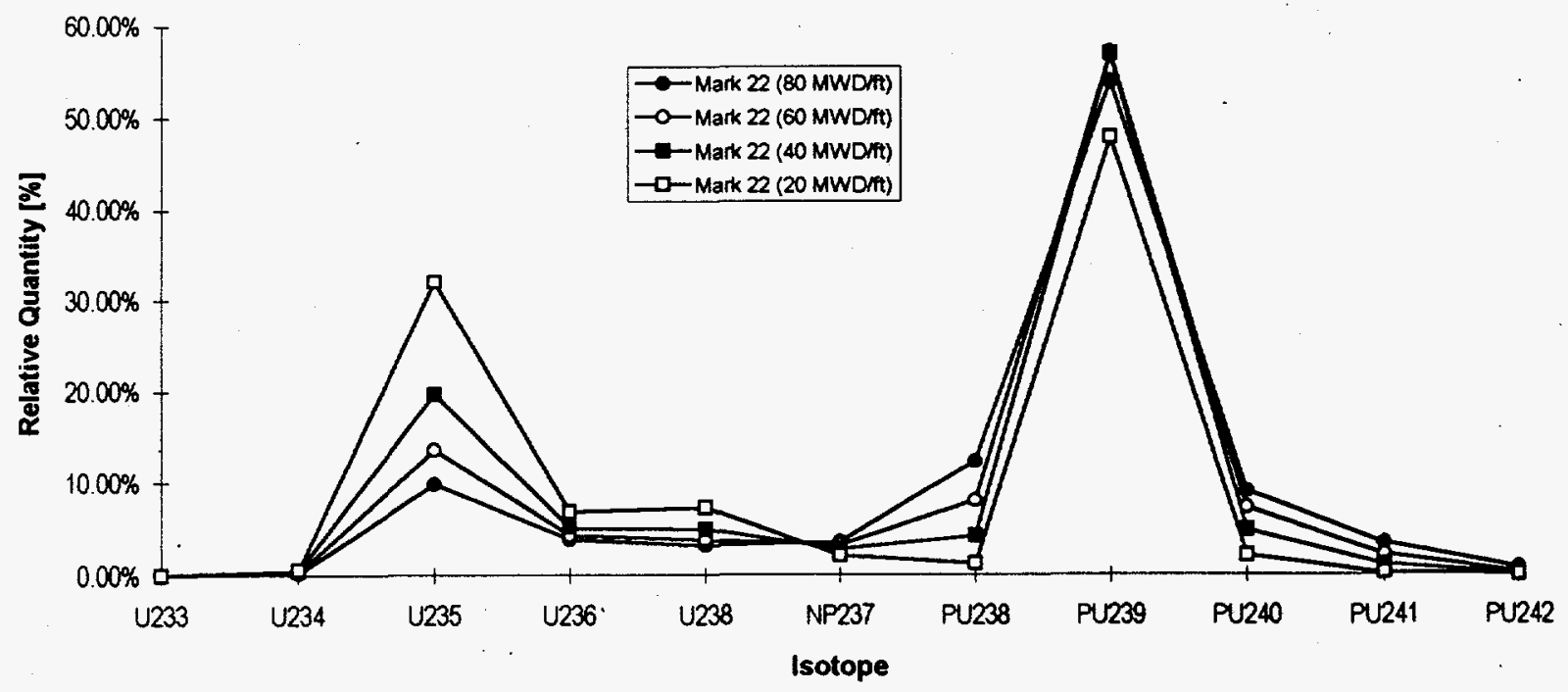


WSRC-TR-94-0456

Revision 0

Page 35 of 56

Figure D-4

Radionuclides in Mark 16B Sludge At Various Exposures

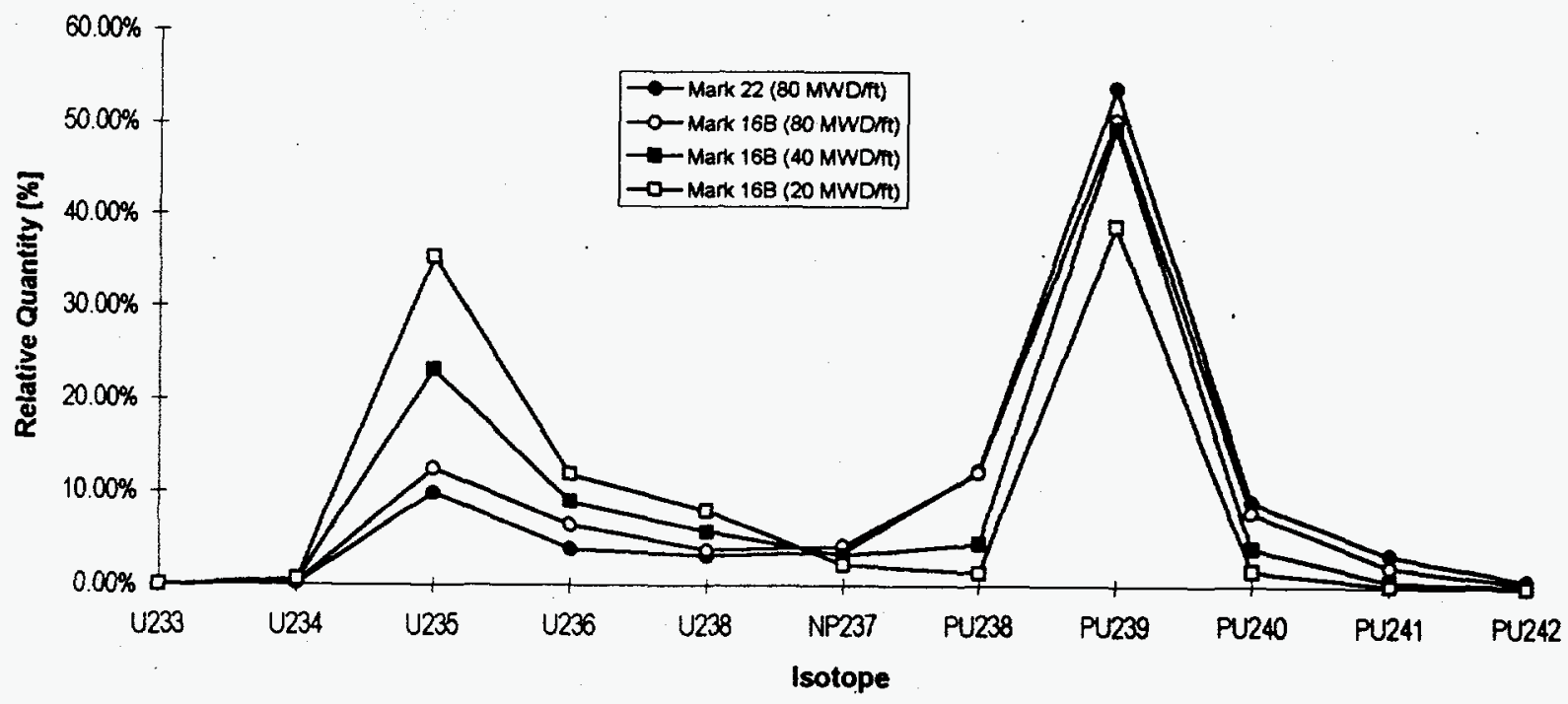


WSRC-TR-94-0456

Revision 0

Page 36 of 56

Figure D-5

Radionuclides in Mark 5E Sludge At Various Exposures

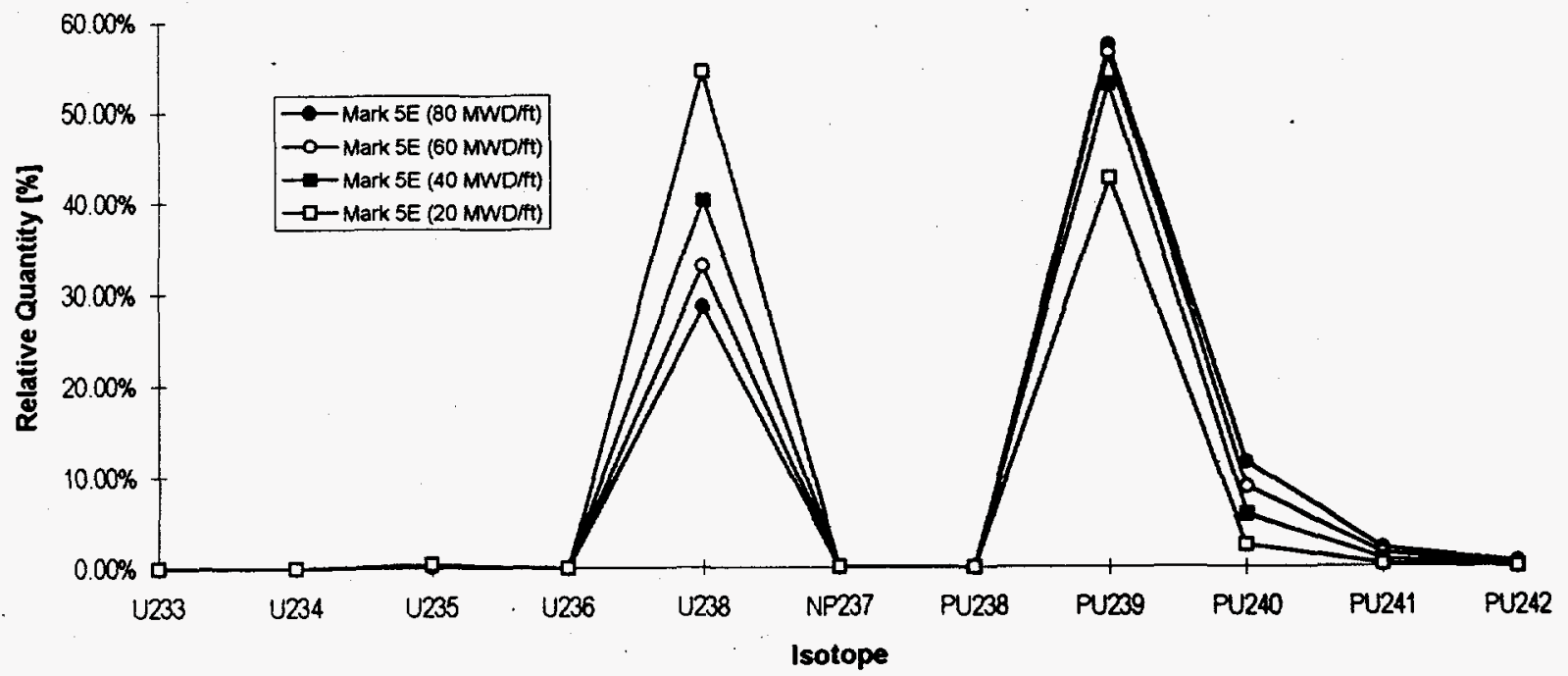


WSRC-TR-94-0456

Revision 0

Page 37 of 56

Figure D-6

Radionuclides in Mark 12A Sludge At Various Exposures

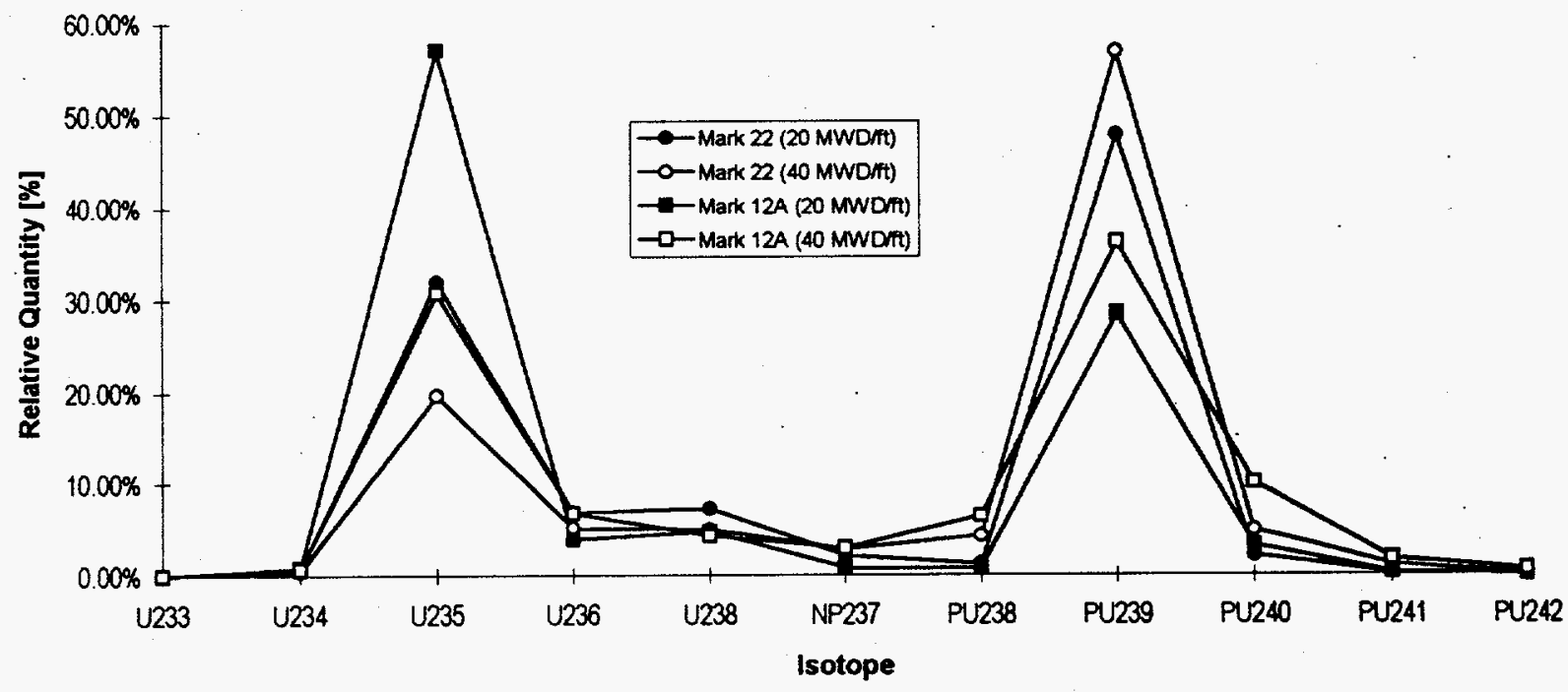


WSRC-TR-94-0456

Revision 0

Page 38 of 56

Figure D-7

Radionuclides in Tank 35H Sludge Versus Mark 16B At Various Exposures

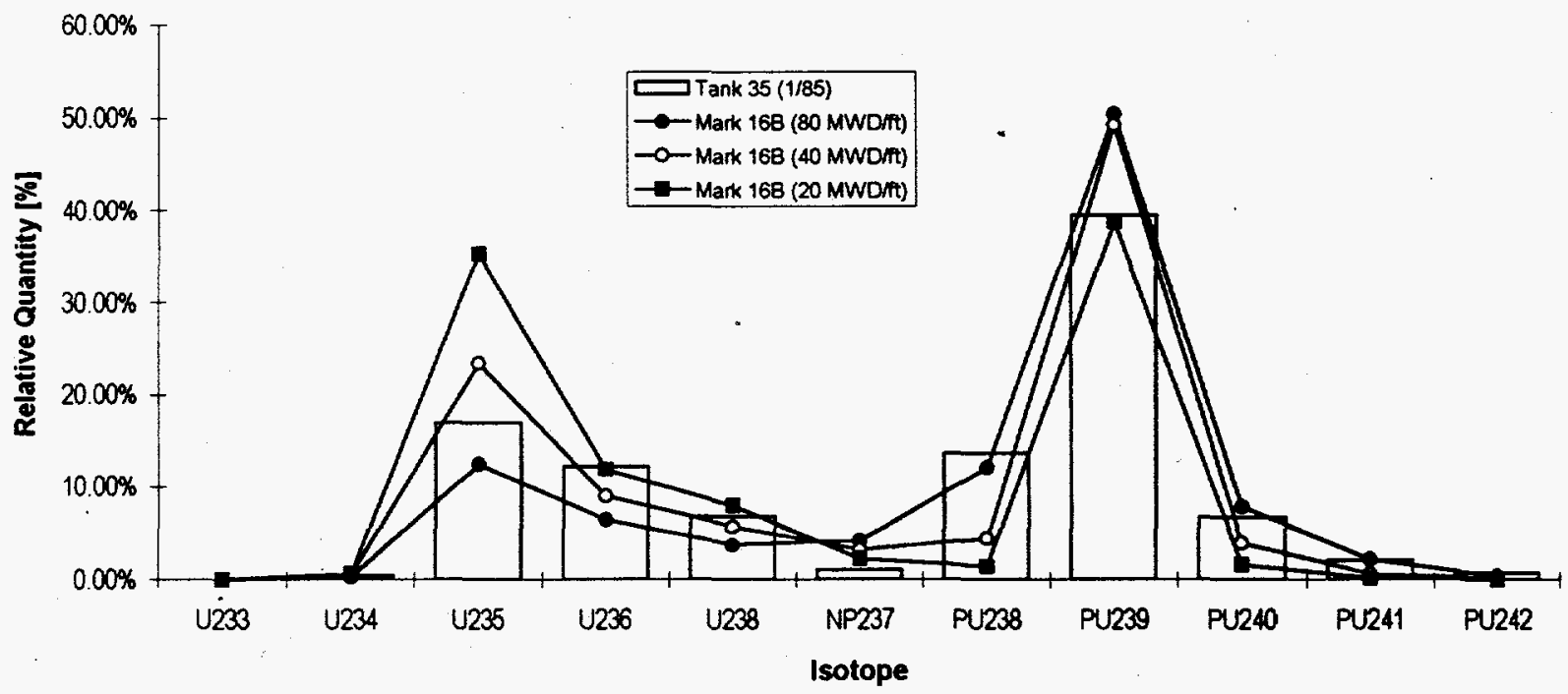


WSRC-TR-94-0456

Revision 0

Page 39 of 56

Figure D-8

Radionuclides in Tank 11H Sludge Versus Mark 22 At Various Exposures

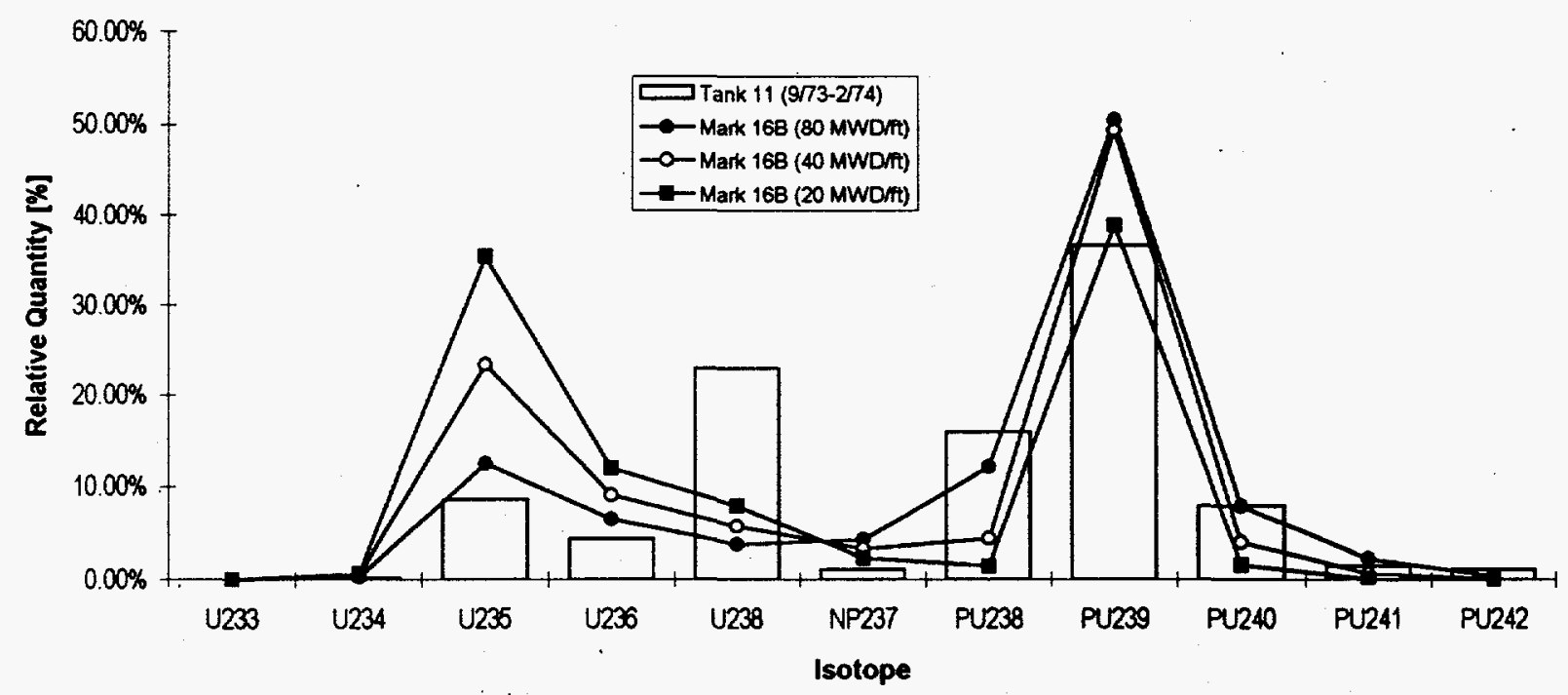


WSRC-TR-94-0456

Revision 0

Page 40 of 56

Figure D-9

Radionuclides in Tank 39H Sludge Versus Mark 16B At Various Exposures

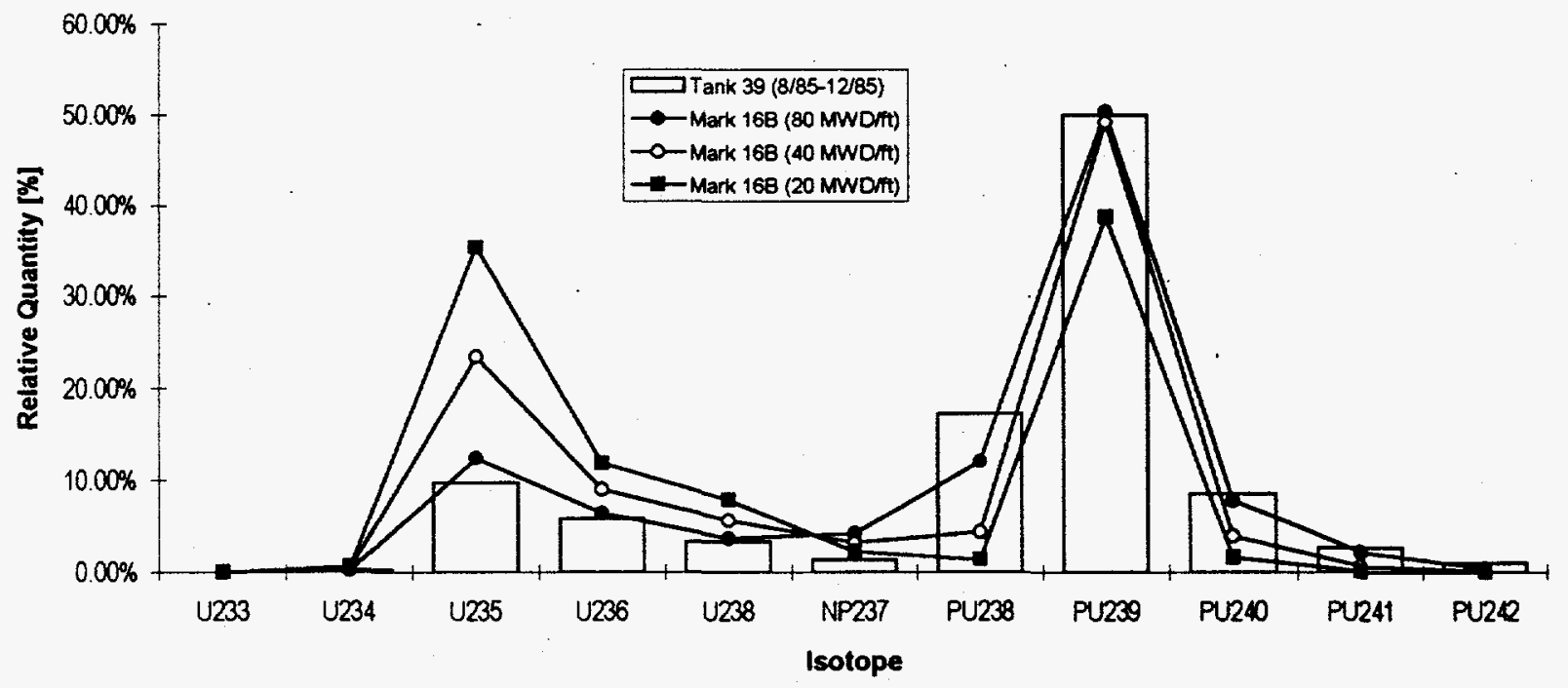


WSRC-TR-94-0456

Revision 0

Page 41 of 56

Figure D-10

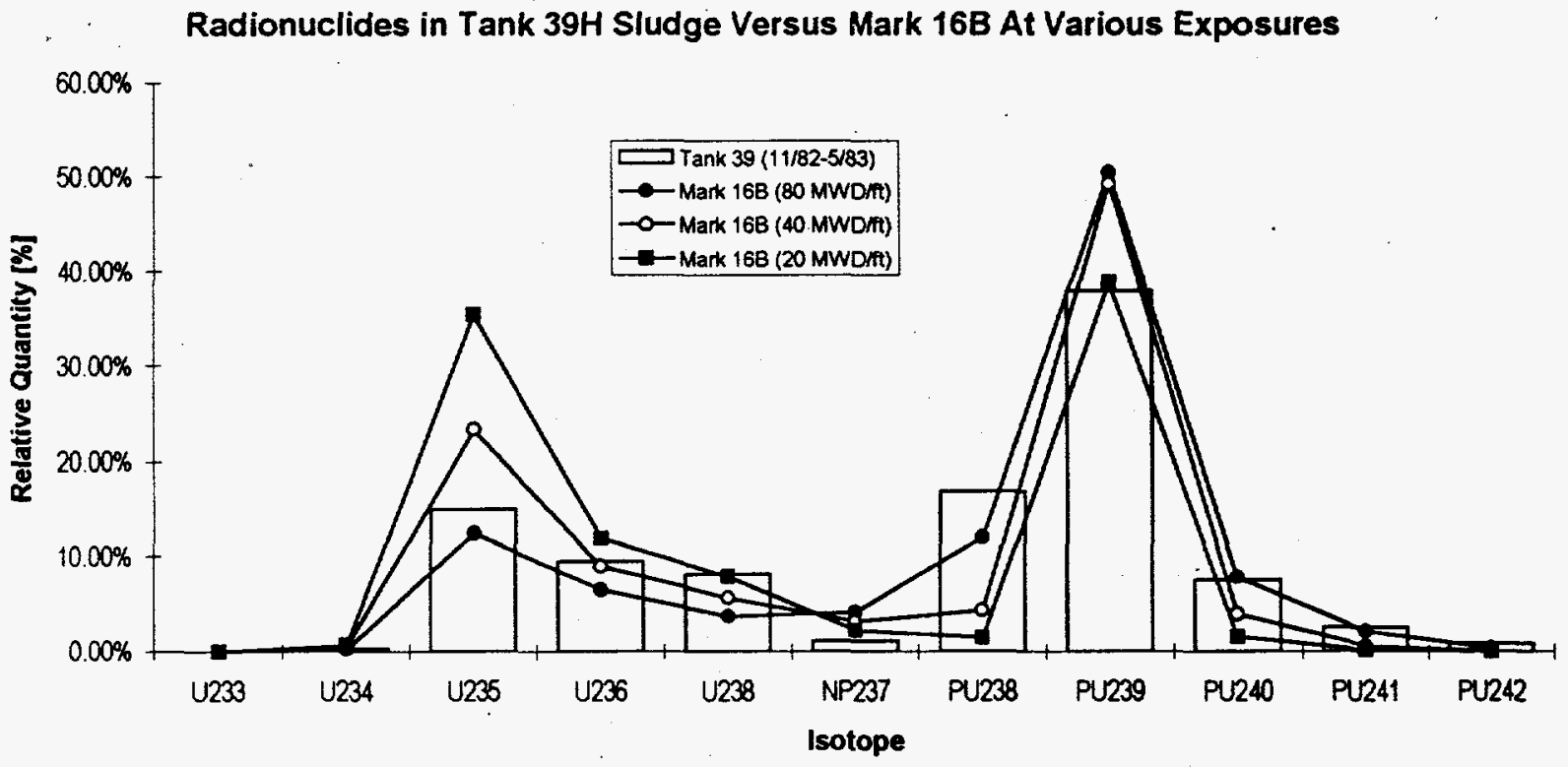


WSRC-TR-94-0456

Revision 0

Page 42 of 56

Figure D-11

Radionuclides in Tank 15H Sludge Versus Mark 22 At Various Exposures

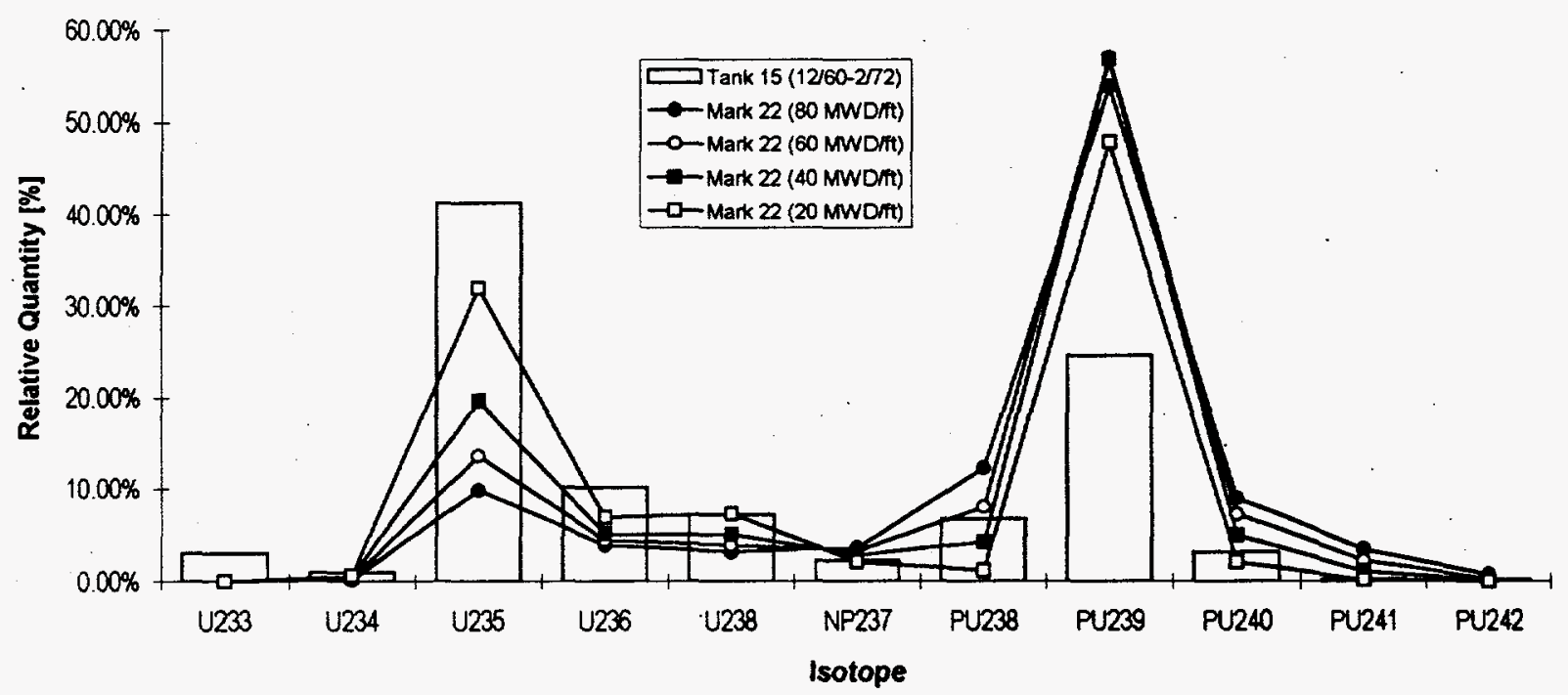


WSRC-TR-94-0456

Revision 0

Page 43 of 56

Figure D-12

Radionuclides in Tank 30H Sludge Versus Mark 22 At Various Exposures

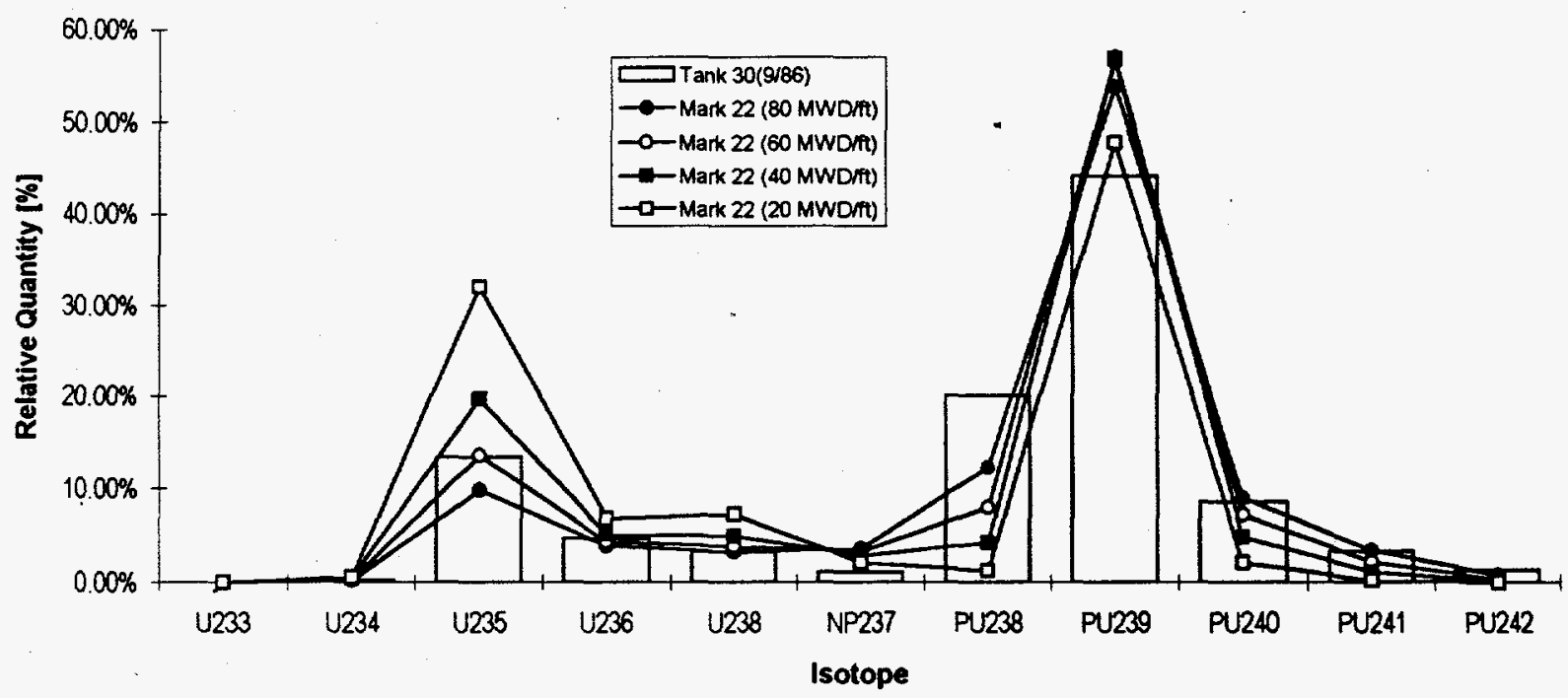


WSRC-TR-94-0456

Revision 0

Page 44 of 56

Figure D-13

Radionuclides in Tank 32H Sludge Versus Mark 22 At Various Exposures

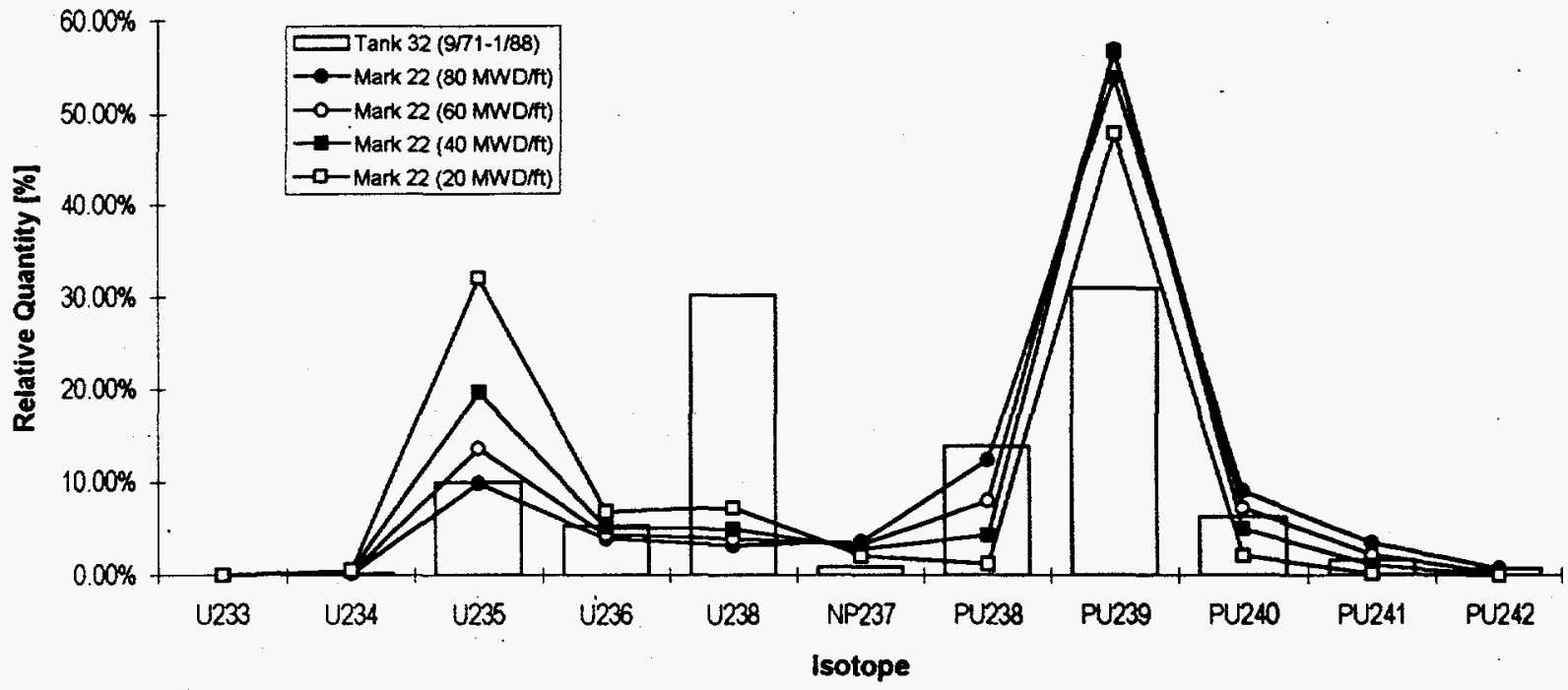


WSRC-TR-94-0456

Revision 0

Page 45 of 56

Figure D-14

Radionuclides in Tank 35H Sludge Versus Mark 22 Sludge At Various Exposures

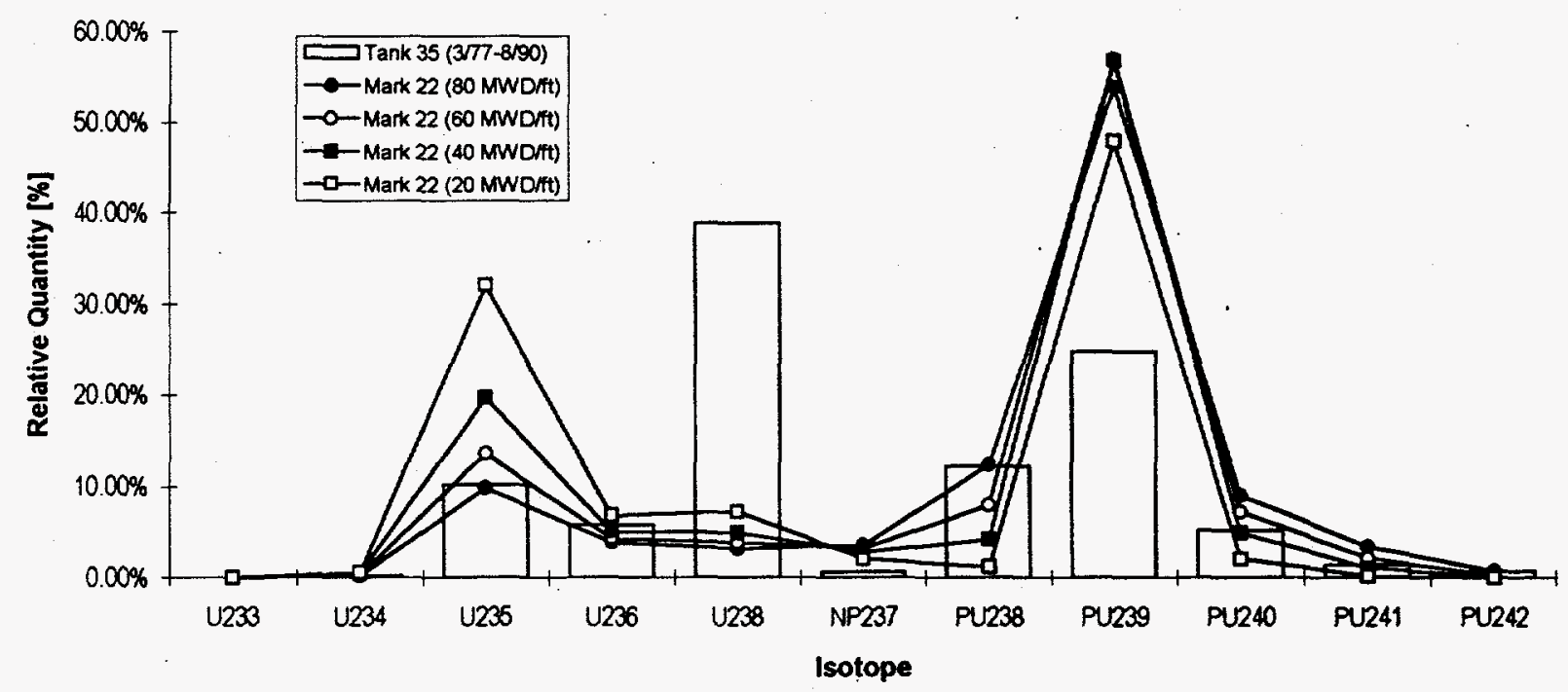


WSRC-TR-94-0456

Revision 0

Page 46 of 56

Figure D-15

Radionuclides in Tank 36H Sludge Versus Mark 22 At Various Exposures

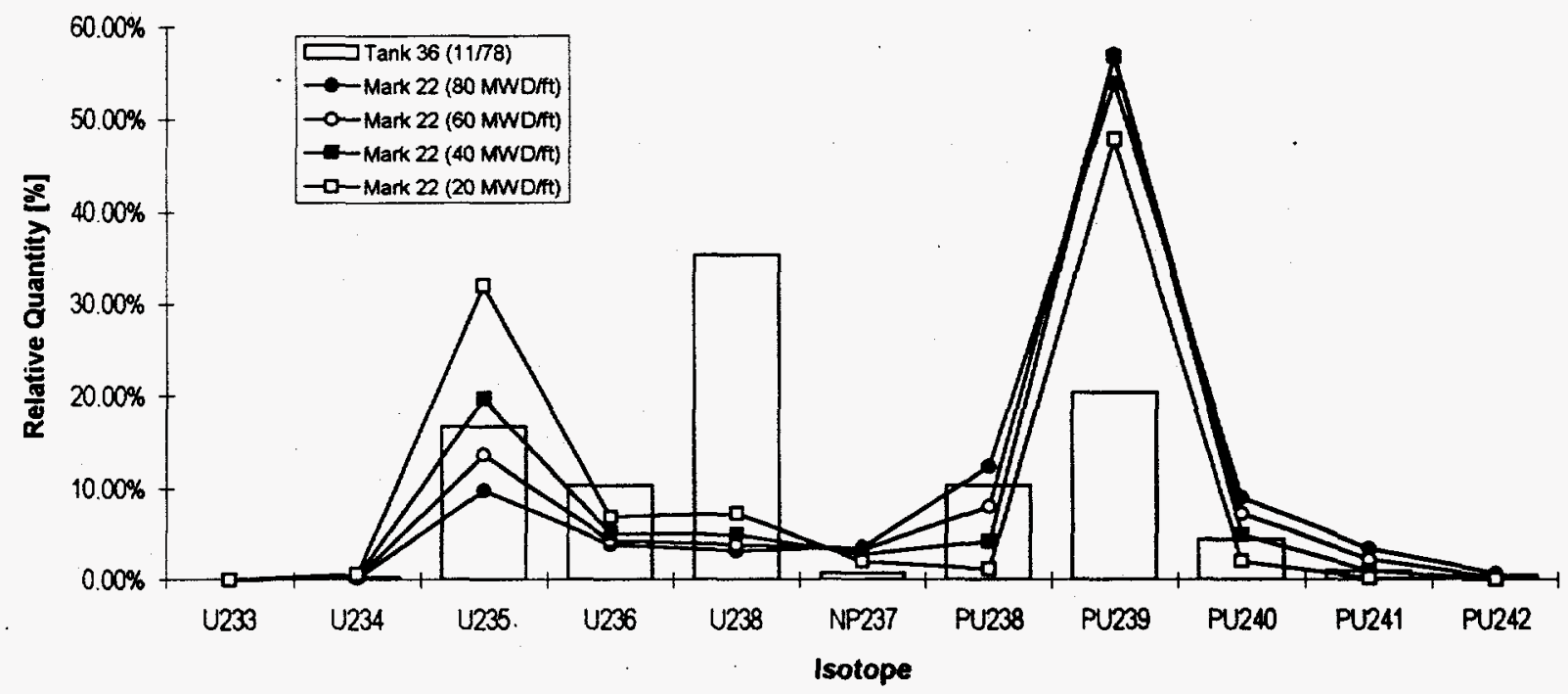


WSRC-TR-94-0456

Revision 0

Page 47 of 56

Figure D-16

Radionuclides in Tank 39H Sludge Versus Mark 22 At Various Exposures

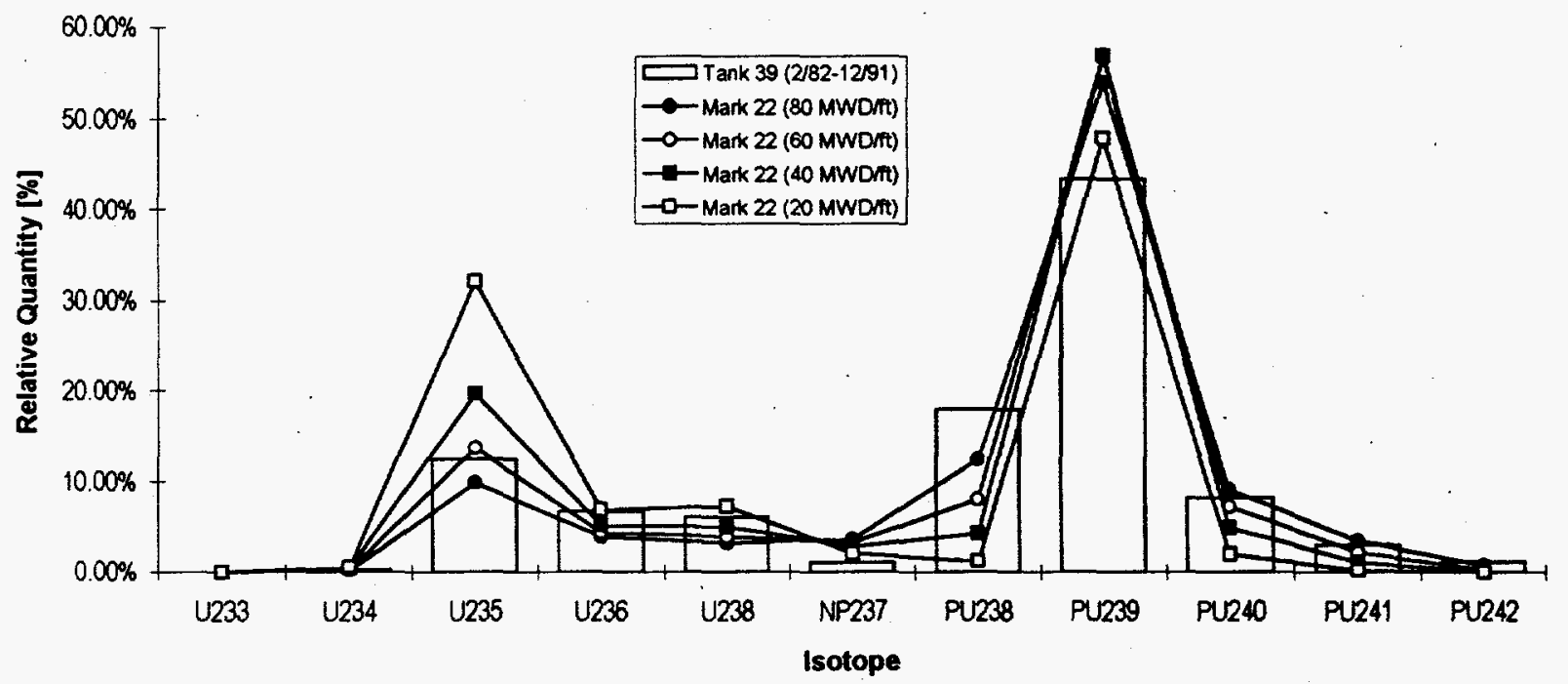


WSRC-TR-94-0456

Revision 0

Page 48 of 56

Figure D-17

Radionuclides in Tank 11H Sludge Versus Mark 22 At Various Exposures

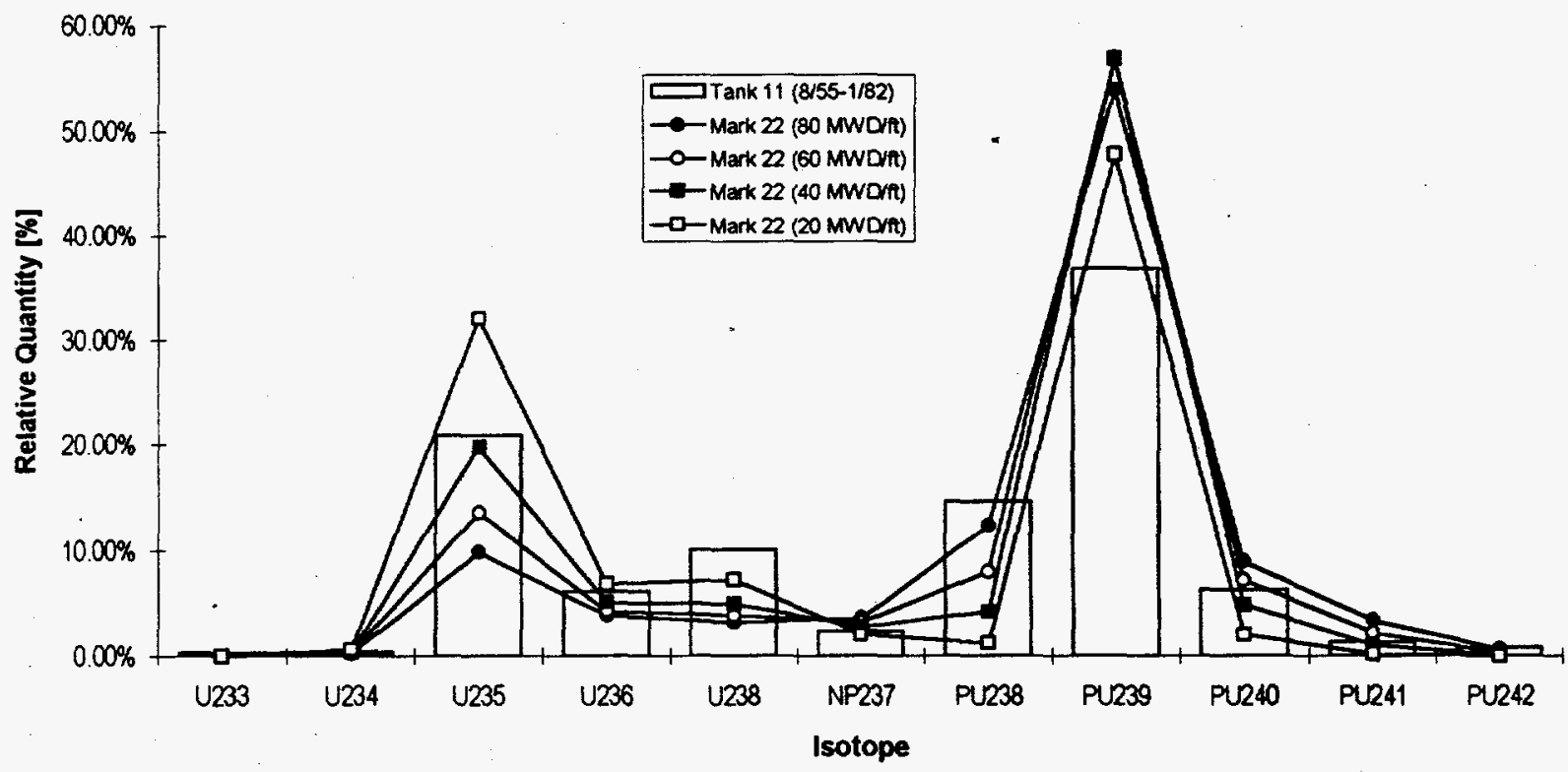


WSRC-TR-94-0456

Revision 0

Page 49 of 56

Figure D-18

Radionuclides in Tank 12H Sludge Versus Mark 22 At Various Exposures

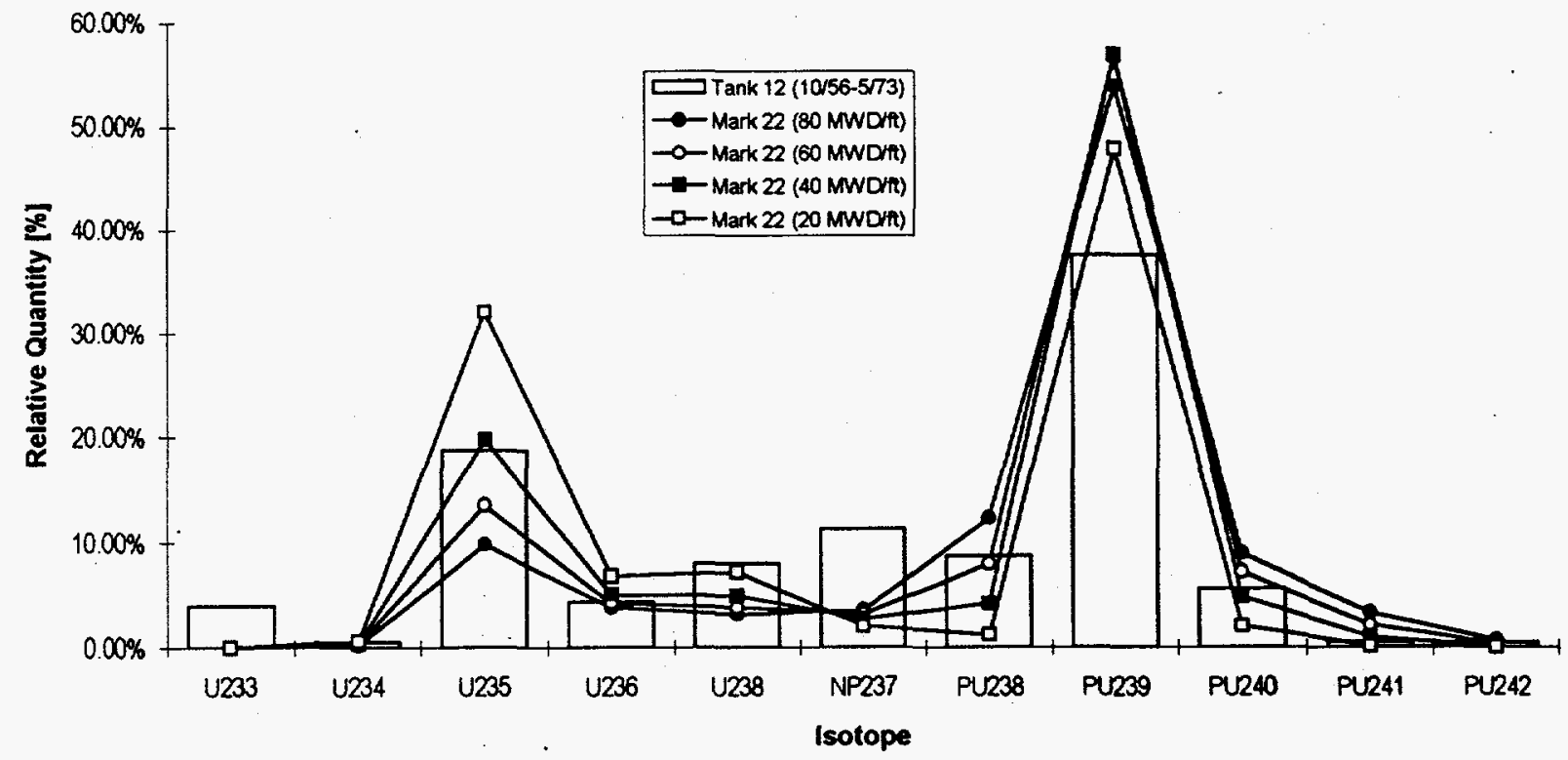


WSRC-TR-94-0456

Revision 0

Page 50 of 56

Figure D-19

Radionuclides in Tank 13H Sludge Versus Mark 22 At Various Exposures

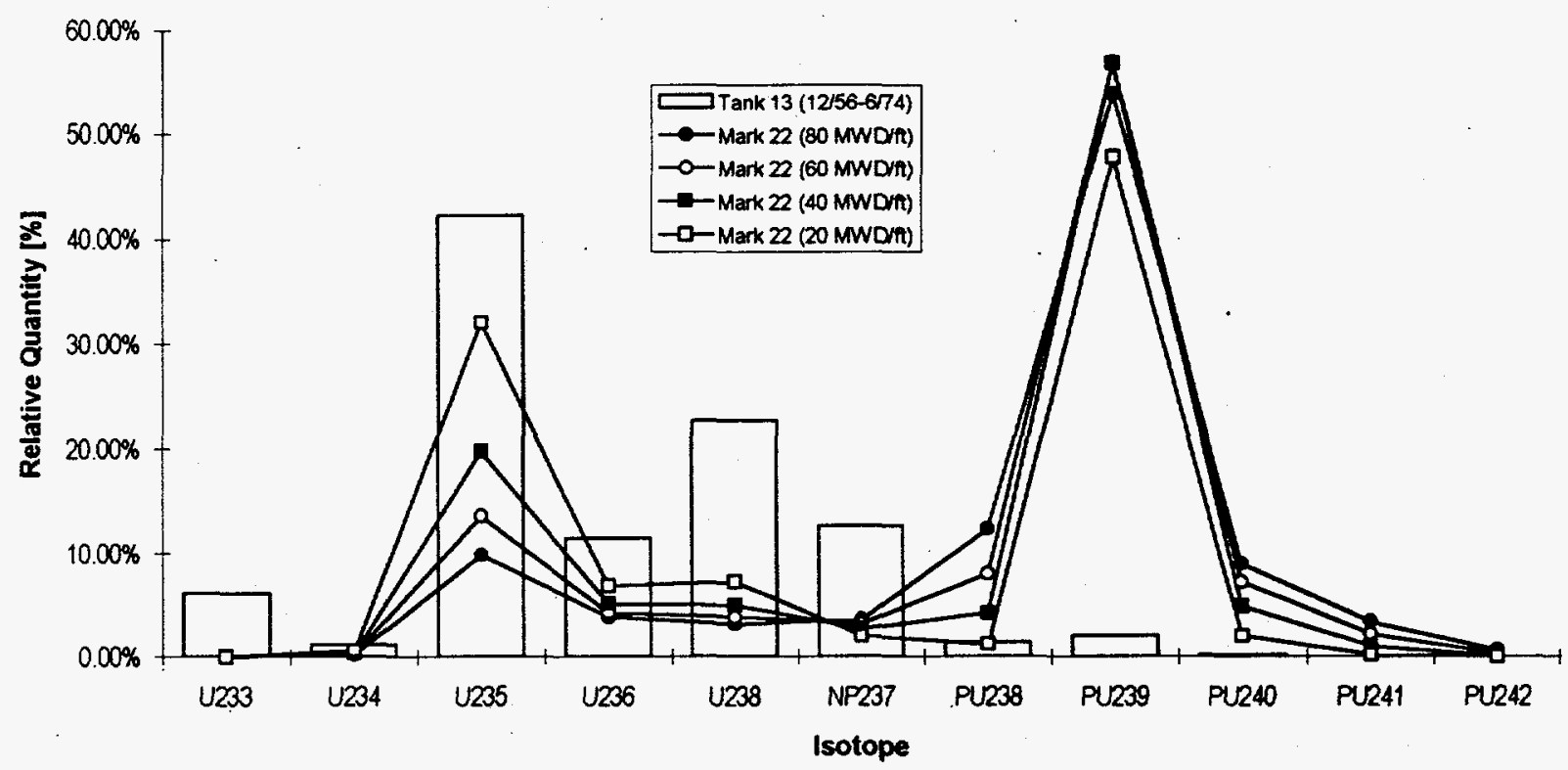


WSRC-TR-94-0456

Revision 0

Page 51 of 56

Figure D-20

Radionuclides in Tank 14H Sludge Versus Mark 22 At Various Exposures

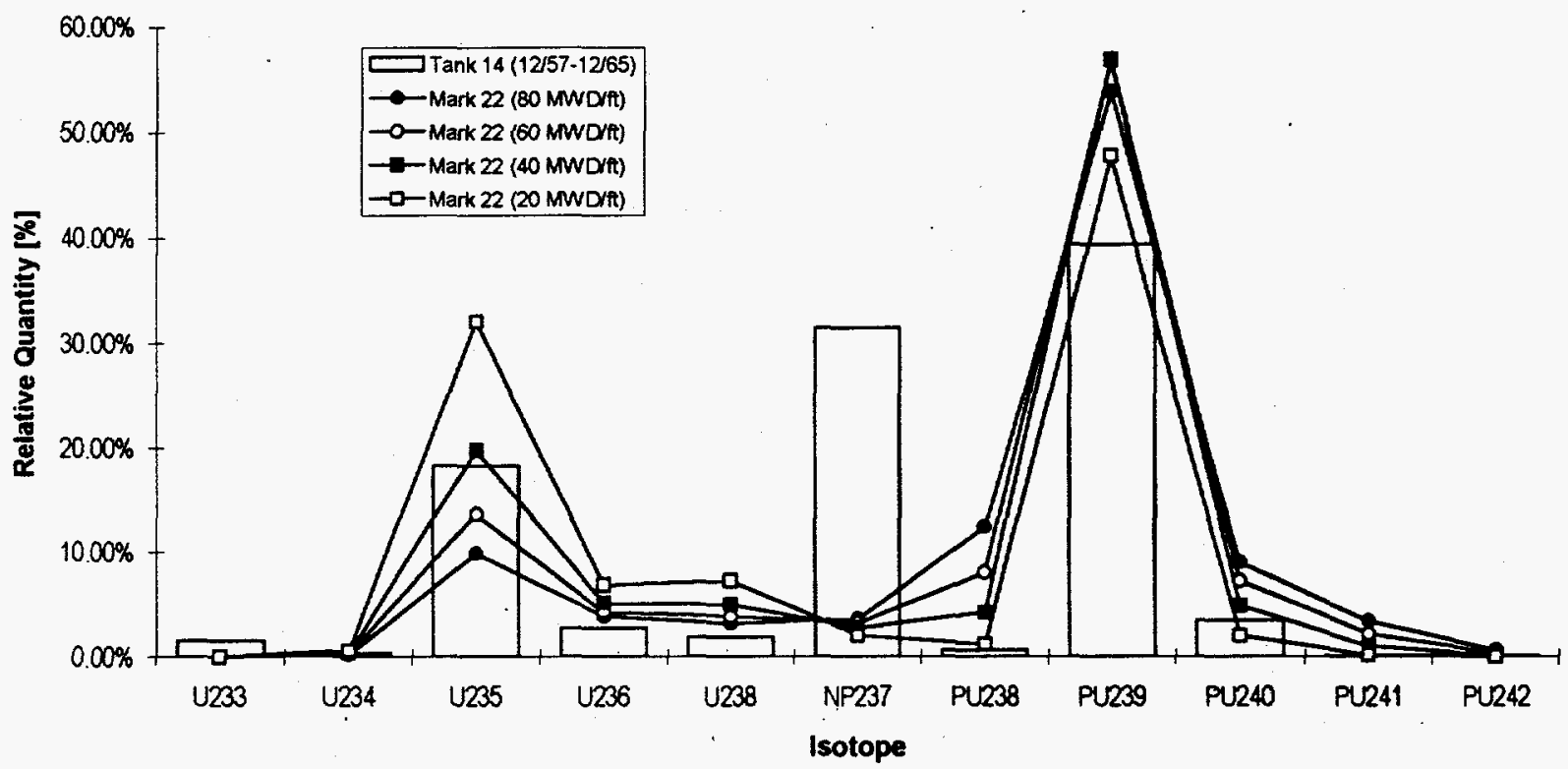


WSRC-TR-94-0456

Revision 0

Page 52 of 56

Figure D-21

Radionuclides in Tank 16H Sludge Versus Mark 22 At Various Exposures

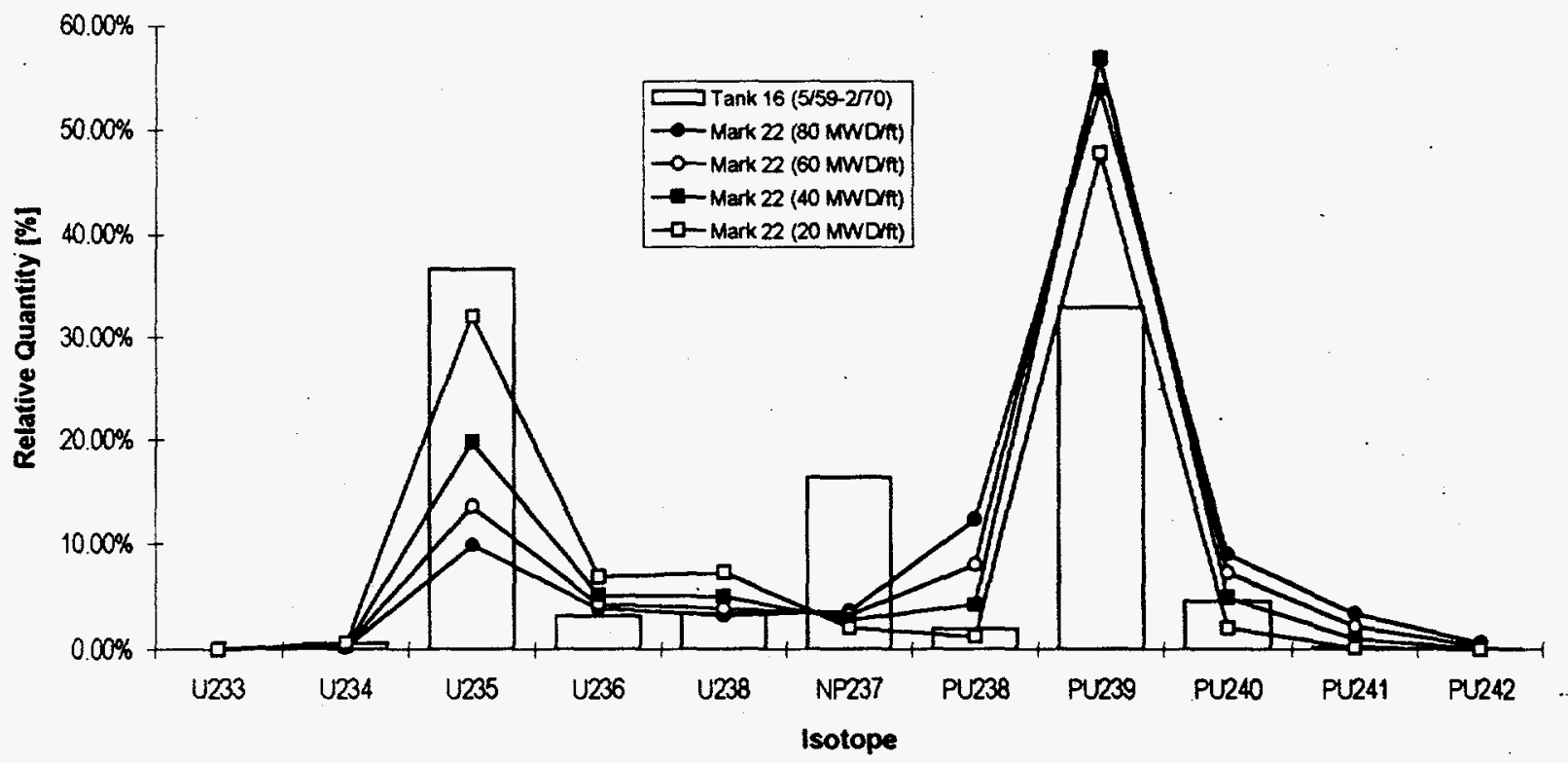


WSRC-TR-94-0456

Revision 0

Page 53 of 56

Figure D-22

Radionuclides in Tank 21H Sludge Versus Mark 22 At Various Exposures

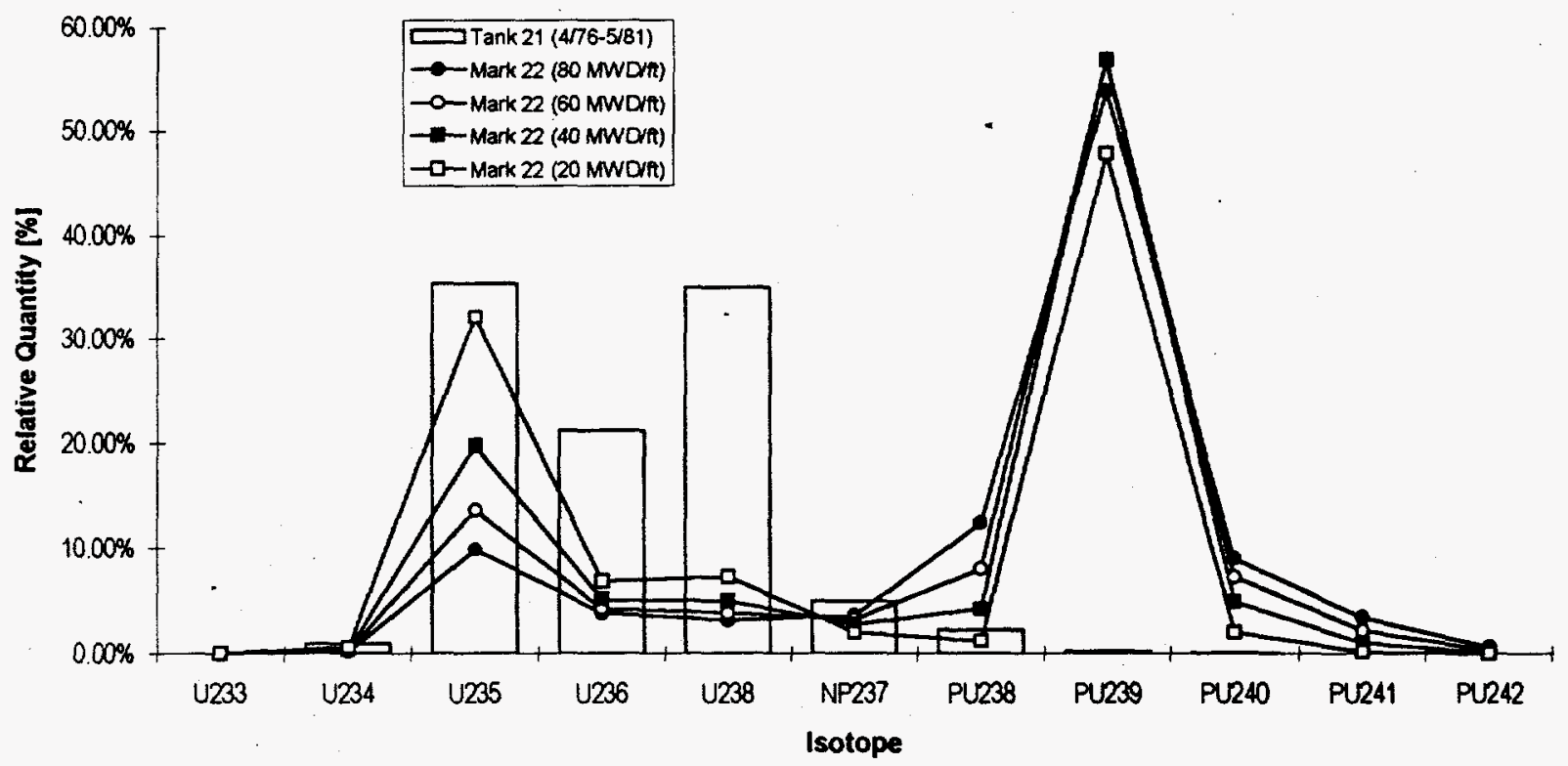


WSRC-TR-94-0456

Revision 0

Page 54 of 56

Figure D-23

Radionuclides in Tank 22H Sludge Versus Mark 22 At Various Exposures

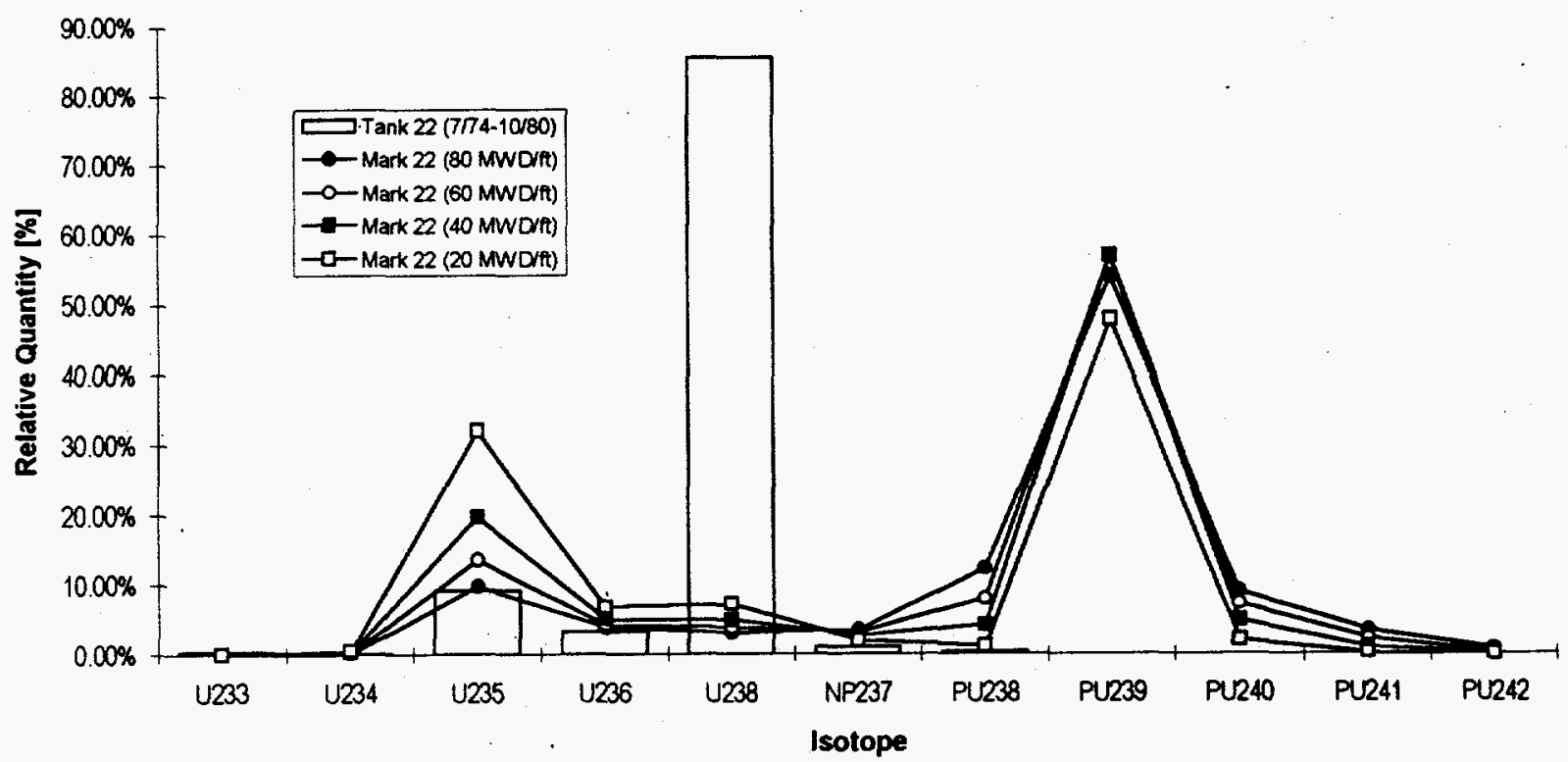


WSRC-TR-94-0456

Revision 0

Page 55 of 56

Figure D-24

Radionuclides in Tank 43H Sludge Versus Mark 22 At Various Exposures

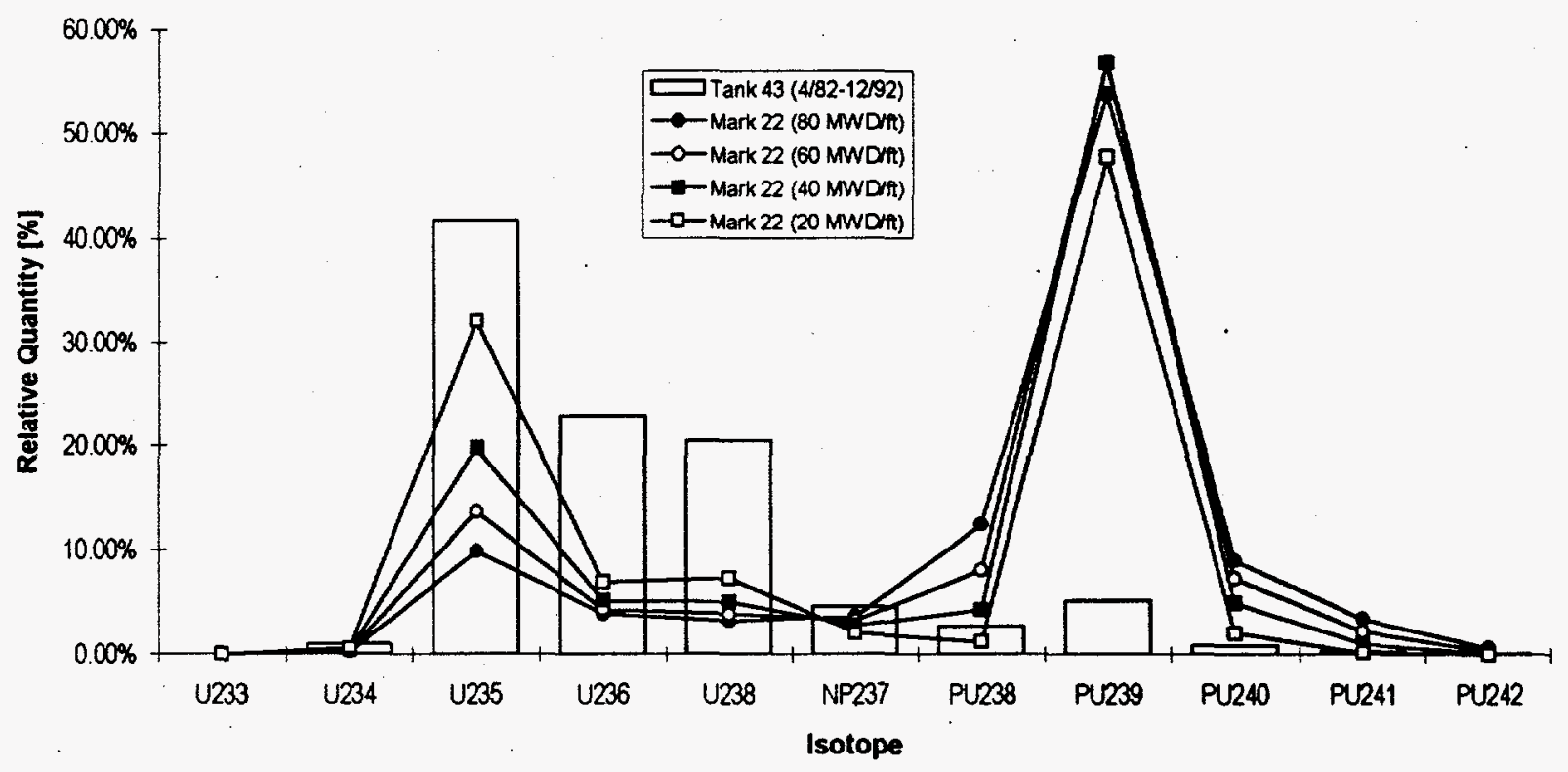


Figure D-25

Radionuclides in Tank 15H Sludge Vs. Mark 16B \& Mark 12A At Exposures Shown

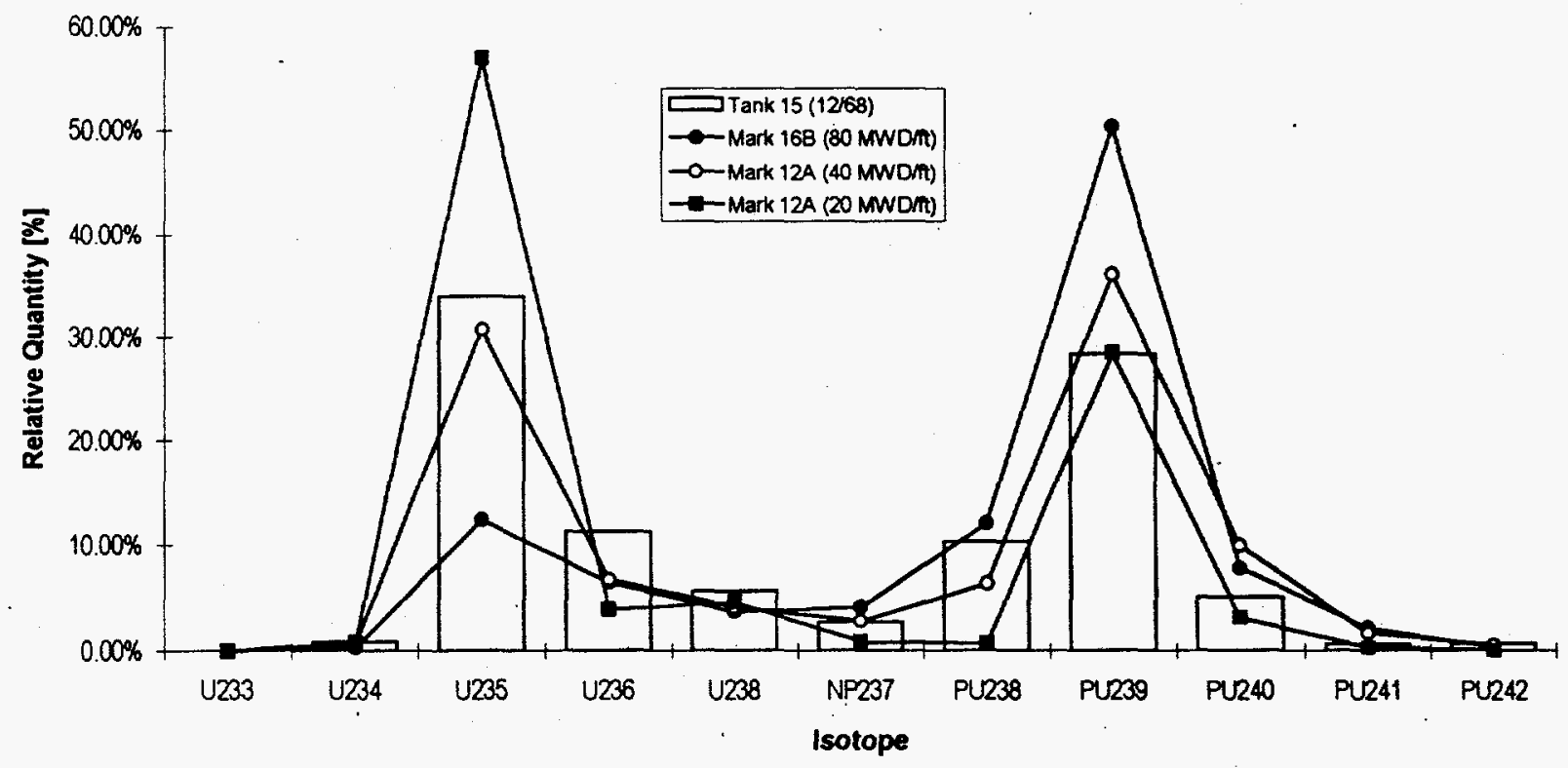

